\title{
Structural properties of self-organized semiconductor nanostructures
}

\author{
J. Stangl* \\ Institute for Semiconductor and Solid State Physics, Johannes Kepler University Linz, \\ A-4040 Linz, Austria
}

\author{
V. Holý ${ }^{\dagger}$ \\ Institute for Condensed Matter Physics, Masaryk University, 61137 Brno, Czech Republic \\ G. Bauer \\ Institute for Semiconductor and Solid State Physics, Johannes Kepler University, Linz, \\ A-4040 Linz, Austria
}

(Published 28 September 2004)

\begin{abstract}
Instabilities in semiconductor heterostructure growth can be exploited for the self-organized formation of nanostructures, allowing for carrier confinement in all three spatial dimensions. Beside the description of various growth modes, the experimental characterization of structural properties, such as size and shape, chemical composition, and strain distribution is presented. The authors discuss the calculation of strain fields, which play an important role in the formation of such nanostructures and also influence their structural and optoelectronic properties. Several specific materials systems are surveyed together with important applications.
\end{abstract}

\section{CONTENTS}

I. Introduction

A. Historical overview

B. Growth mechanisms

II. Description of Growth Phenomena

A. Step-flow growth

1. Surface reconstruction

2. Step bunching

3. Step meandering

4. Lateral segregation in short-period superlattices

726

726

726

727

727

728

729

730

5. Nucleation and growth of two-dimensional islands

B. Stranski-Krastanow growth

1. Asaro-Tiller-Grinfeld instability

2. Island nucleation

3. Island shapes

4. Interdiffusion and segregation

5. Scaling of the island sizes

6. Dislocations

7. Changes of shape, strain, and composition during overgrowth

C. Correlation of quantum dot positions

1. Lateral island correlation

2. Vertical island correlation

3. Oblique correlation

4. Self-organization process on prepatterned substrates

III. Experimental Methods

\footnotetext{
*Electronic address: julian.stangl@jku.at

${ }^{\dagger}$ Present address: Department of Electronic Structures, Charles University, Prague, Czech Republic.
}

A. Atomic force microscopy

B. Scanning tunneling microscopy and cross-sectional STM

C. Transmission electron microscopy and scanning electron microscopy

1. Morphology

2. Digital analysis of lattice images

3. Composition evaluation by lattice fringe analysis

4. Quantitative analysis of the information from transmission electron micrographs $\quad 748$

5. Energy-selective imaging 749

6. Scanning transmission electron microscopy $\quad 749$

D. Low-energy electron microscopy 749

E. X-ray scattering 750

1. Scattering theory 751

2. Grazing-incidence small-angle x-ray scattering 752

3. X-ray reflectivity 754

4. Coplanar X-ray diffraction 754

5. Grazing-incidence x-ray diffraction 756

6. Anomalous x-ray scattering 759

7. Coherent diffraction 759

8. Extended x-ray absorption fine structure $\quad 760$

F. Optical methods $\quad 760$

1. Raman scattering 761

2. Photoluminescence 761

3. Investigations of single quantum dots 763

IV. Calculation of the Strain Distribution Within and Around Nanostructures $\quad 763$

A. Analytical methods in continuum elasticity $\quad 763$

B. Numerical methods in continuum elasticity $\quad 766$

C. Atomistic simulations $\quad 767$

V. Important Materials Systems $\quad 769$
A. $\mathrm{SiGe}$ on $\mathrm{Si}$ 


\author{
B. InAs on GaAs \\ C. $\mathrm{GaN}$ \\ D. InAs on InP \\ E. InP and GaInP \\ F. ErAs and $\mathrm{ErSb}$ \\ G. CdSe \\ H. $\mathrm{PbSe}$ \\ VI. Applications \\ A. Optical applications: Detectors and lasers \\ B. New concepts \\ VII. Outlook and Prospects \\ Note added in proof \\ Acknowledgments \\ References \\ 770 \\ 771 \\ 771 \\ 771 \\ 772 \\ 772 \\ 772 \\ 773 \\ 773 \\ 773 \\ 775 \\ 776 \\ 776 \\ 776
}

\section{INTRODUCTION}

Two main factors have driven the increasing attention received by semiconductor nanostructures in the last decade: first, they are attractive from a scientific point of view, since they provide a means to create artificial potentials for carriers, electrons, and holes in semiconductors, at length scales comparable to or smaller than the de Broglie wavelength. Consequently, quantum confinement effects become not only important, but also designable to a large degree. Many concepts that previously existed merely as simplifying theoretical models can now be practically realized in semiconductor nanostructures, so their properties can be investigated. The second important factor is that quantum mechanics becomes applicable not only in systems of academic interest, but also to systems of practical impact. Using confinement effects, new device concepts become feasible, which receive additional degrees of freedom in design. Restrictions due to material properties are shifted or lifted. In particular, semiconductor nanostructures have a large potential for applications in nano- and optoelectronics.

The first systems resembling a simple theoretical model, namely, that of a one-dimensional potential variation, e.g., a quantum well, were epitaxially grown heterostructures, stacks of planar layers of different semiconductors, which formed a potential variation along the growth direction. The ultimate limit of lowdimensional structures is, however, the quantum dot, in which the carriers are confined in all three dimensions. Numerous attempts have therefore been made to investigate the possibility of lateral patterning of layered systems in order to achieve confinement in all three spatial dimensions. The required structure sizes are on the order of $10 \mathrm{~nm}$ in most semiconductor systems, which is challenging to fabricate with conventional techniques. Methods to achieve sufficiently small structures do exist, e.g., electron-beam lithography, and it has been successfully demonstrated that in principle the concepts work. But another important issue for devices is that the dot interfaces must be kept free of defects in order to obtain the desired electronic properties. The development of fabrication methods that avoid such defects remains difficult.
On the other hand, ways to fabricate small structures in large numbers and without interface defects have been known for a long time, only they have been regarded as a failure of growth rather than as a chance to fabricate quantum dots: Spontaneous undulations and a "roughening" of the growing surface have been perceived as a problem in semiconductor epitaxy, degrading the interfaces between different materials. Around 1990 it became gradually accepted that such a "rough" surface may well be considered as a surface containing nanostructures. It was discovered that the undulations were formed as a way to reduce the stress in the growing film. Obviously, a rough surface is rather far from the conventional idea of a surface of quantum dots of certain, predictable properties. In many cases, the size distribution of self-organized islands is fairly broad, and due to the fact that the island nucleation process is statistical in nature, lateral ordering is typically absent.

A rapidly increasing number of studies has sought to understand, and hence control, self-organizing growth processes, and progress has been made towards producing islands with a narrow size distribution for various combinations of materials. Ways to achieve laterally correlated islands have been discovered, as well, and in some cases self-assembled islands have already made their way from objects of interest in basic science to key components in devices.

\section{A. Historical overview}

In the original paper by Stranski and Krastanow (1938) island formation in heteroepitaxial ionic crystals was investigated. Nowadays this growth mode describes island formation on a wetting layer in heteroepitaxial systems with different lattice constants. In InAs/GaAs superlattices, Goldstein et al. (1985), observed for the first time island formation in a semiconductor system. Originally, this instability leading to island growth was considered detrimental for the growth of quantum well systems in strained heteroepitaxy, and efforts were undertaken to avoid it. However, from 1990 onwards this topic has gained considerable attention, as it was realized that such islands may represent zero-dimensional semiconductor nanostructures (Eaglesham and Cerullo, 1990; Guha et al., 1990; Mo et al., 1990). It was found that, for suitable growth conditions, the size distribution (height and lateral size) could be substantially narrowed, which reduced inhomogeneous line broadening and thus made the determination of optical properties of quantum dots much more reliable. It was soon established that the quasi-zero-dimensional density of states in InAs islands embedded in GaAs could be used to realize devices such as injection lasers, which exhibit properties in some respects superior to strained III-V quantum well systems.

\section{B. Growth mechanisms}

Several methods for the self-organized growth of semiconductor nanostructures have been suggested. 
Among them are rather exotic ones, such as, for instance, the growth of $\mathrm{Ge}$ on amorphous nitride films with small holes, through which an epitaxial relation between Ge islands and the Si substrate is mediated. There are, however, phenomena that are rather universal and can be exploited for a large variety of semiconductor materials. Those phenomena will be discussed in the following sections, reviewing the achievements made and the problems frequently encountered so far. The most prominent growth method is certainly StranskiKrastanow growth, already mentioned above. This mode, leading to the formation of nanoscale islands on top of a two-dimensional (2D) wetting layer, occurs for almost any semiconductor heterostructure with a certain lattice mismatch between the constituent materials. But even in a mode in which no islands form, in layer-bylayer epitaxy, nanostructures may evolve. If $2 \mathrm{D}$ layers grow in a step-flow regime, i.e., by an effective movement of monolayer or bimonolayer steps across the surface, the interaction of those steps can form step bunches. In a multilayer, for instance of $\mathrm{SiGe}$ and $\mathrm{Si}$, preferential agglomeration of Ge at these step bunches can form one-dimensional nanowires. Depending on the direction of the misorientation of the substrate surface with respect to the ideal crystallographic surface orientation, the step bunches may also break up into a zigzag pattern, and even in homoepitaxy small zerodimensional structures may arise.

The strain distribution in a growing sample is one of the main driving forces leading to the formation of nanostructures, and it also influences the particularities of their growth and mutual arrangement. This can lead to very regular ensembles of nanostructures, with narrow size distribution, lined up in more or less perfect one-, two-, or even three-dimensional lattices. A main effect here is that the strain field of, say, a small buried island influences the nucleation of islands at the growth surface, even if the surface undulations due to the buried structure have already vanished.

This article is intended to give a comprehensive overview of the mentioned growth modes, and of the methods that are employed to investigate and characterize semiconductor nanostructures. It is understood that such a review cannot be complete. Rather, we intend to give an introduction stressing several points that we believe have not been discussed in great detail in existing reviews. Electronic and optical properties, as well as devices, will be mentioned, but are not the focus of this review. For reviews mainly on the growth of nanostructures, the reader is referred to Notzel (1996), Petroff and Medeiros-Ribeiro (1996), Seifert et al. (1996), and Brunner (2002). Recently, a monograph by Shchukin et al. (2003) dealt extensively with growth, self-organization phenomena, and devices based on nanostructures.

Theoretical calculations of the elastic properties of nanostructures are reviewed by Gao (1994), Zunger (1994), and Shchukin and Bimberg (1999). For optical and electronic properties, see Seifert et al. (1996), Grundmann et al. (1998), Lueth (1998), Bimberg et al. (1999), Moriarty (2001) and Brunner (2002). Reviews on the structural properties of SiGe nanostructures, with the main emphasis on characterization by atomic force microscopy, were presented by Liu and Lagally (1997) and Teichert et al. (2002). Two issues of the Materials Research Society Bulletin, Vol. 21/4 (1996) and Vol. 23/2 (1998), are devoted to issues of heteroepitaxy, strain, growth, spectroscopy, laser applications, and bandstructure calculations of quantum dots. Petroff et al. (2001), Gammon and Steele (2002), and Grundmann (2002) review the potential applications in optical and optoelectronic devices exploiting the atomlike electronic properties of quantum dots.

This review will focus on the structural properties of nanostructures fabricated by various techniques, from molecular-beam epitaxy to various chemical vapor deposition techniques to liquid-phase epitaxy. The different growth models are discussed in some detail, and we present the most important characterization techniques, such as atomic force microscopy (AFM), scanning tunneling microscopy (STM), transmission electron microscopy (TEM), x-ray scattering, Raman scattering, and photoluminescence, particularly stressing $\mathrm{x}$-ray scattering methods. As strain fields in and around nanostructures are a driving force in their growth and important for the interpretation of experimental results, a full section is devoted to methods of calculating them.

In order to achieve a consistent description of growth modes and characterization techniques, we shall focus mainly on the SiGe and InGaAs systems as the most prominent ones. Other important materials systems will be described and their similarities and differences, highlighted. We shall also discuss the main applications of each model system.

\section{DESCRIPTION OF GROWTH PHENOMENA}

\section{A. Step-flow growth}

The driving force for the self-organization processes during heteroepitaxial growth is the misfit between the crystal lattice of the growing layer and that of the substrate, which creates strain in the growing layer. In the very first stage of growth, a pseudomorphic thin layer is created, which is elastically distorted. During growth the elastic energy stored in this layer increases. In the linear continuum model, the volume density of this energy is proportional to the square of the elastic tensor $\varepsilon_{j k}$ as $E_{\mathrm{el}}=\frac{1}{2} c_{j k m n} \varepsilon_{j k} \varepsilon_{m n}$.

In general, either plastic or elastic relaxation of this internal elastic energy is possible. During plastic relaxation, the elastic energy is reduced by misfit dislocations at the substrate/layer interface (Matthews and Blakeslee, 1974; Dodson and Tsao, 1987); this process competes with elastic relaxation in leading to selforganized nanostructures. The latter mechanism is the topic of this review. 
(a) unreconstructed $\stackrel{L}{\longrightarrow}[\overline{1} 10]$ reconstructed
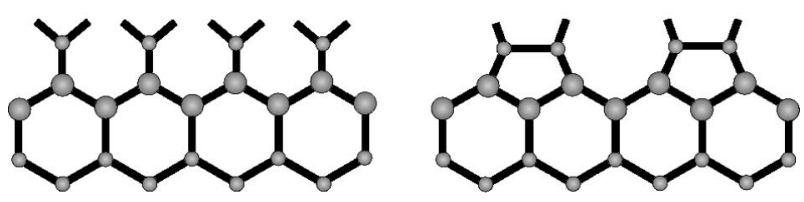

(b)

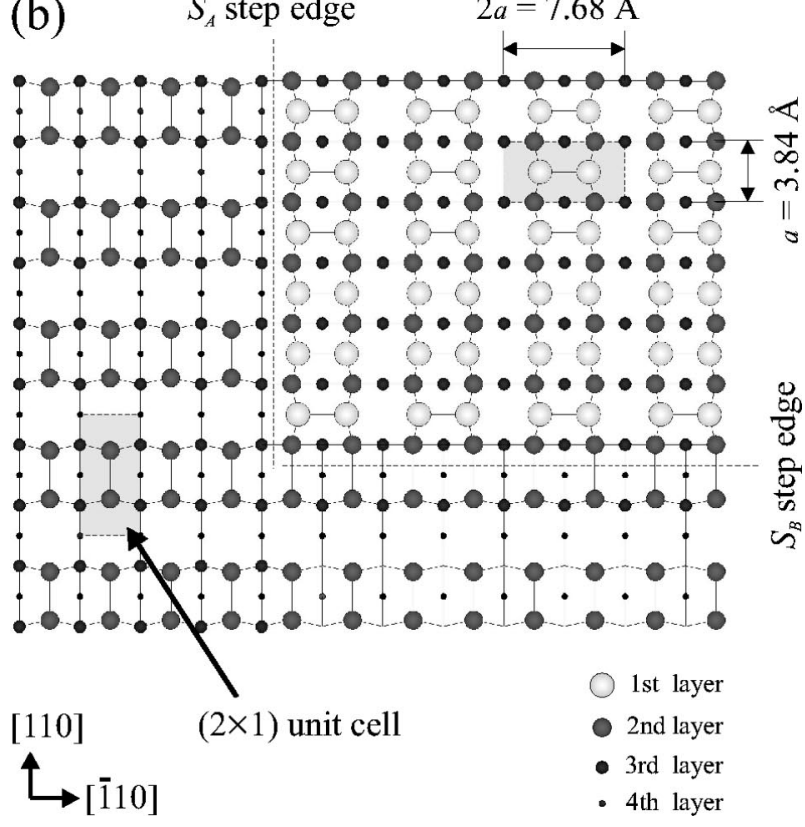

FIG. 1 . The $2 \times 1$ surface reconstruction of $\mathrm{Si}(001)$ : top panel, side view; lower panel, top view. In the lower panel, $S_{\mathrm{A}}$ and $S_{\mathrm{B}}$ steps are sketched parallel and perpendicular to the dimer rows, respectively. After Voigtländer, 2001 with permission from Elsevier. Copyright 2001.

\section{Surface reconstruction}

Elastic energy relief is important for the formation of self-assembled nanostructures, and this in turn depends substantially on layer thickness: For layer thicknesses below and about 1 monolayer (ML), the elastic energy is reduced by surface reconstruction (see, for instance, Zangwill, 1988). On the $\mathrm{Si}(001)$ surface, a $2 \times 1$ surface reconstruction is observed, as shown in Fig. 1. The reconstructed dimer bonds are oriented parallel to the $\langle 110\rangle$ direction. In practice, a surface normal will not be aligned perfectly along a crystallographic direction, e.g., [001], but will have a finite misorientation or miscut, with a magnitude of typically $0.1^{\circ}-4^{\circ}$. Consequently the surface consists of a sequence of atomically flat (001) terraces separated by monolayer steps. The dimer orientation at neighboring terraces is mutually perpendicular.

If a $2 \times 1$-reconstructed $\mathrm{Si}(001)$ surface is covered by a Ge layer of submonolayer thickness, the incorporation of Ge leads first to the formation of so-called "buckled dimers," as shown in Fig. 2 (Qin et al., 2000b). For increasing Ge coverage, the surface reconstruction type
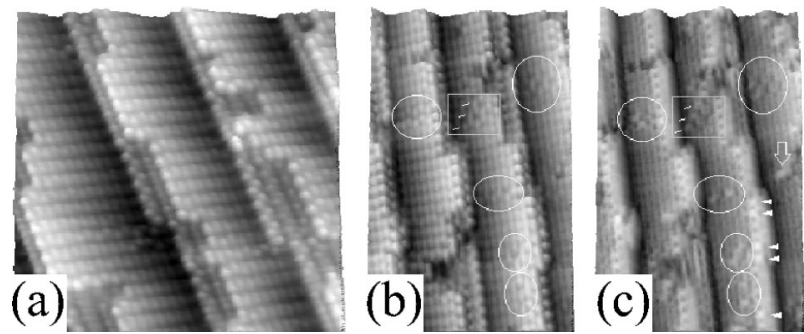

FIG. 2. Scanning tunneling microscope (STM) images of $\mathrm{Si}(001)$ with $4^{\circ}$ miscut in the [110] direction prior and after $\mathrm{Ge}$ deposition: (a) filled state of pure $\mathrm{Si}$; (b) filled state after deposition of 0.02 ML's of Ge; (c) empty-state image of the area shown in (b). Ge incorporation leads to the formation of buckled dimer rows (indicated by boxes and ovals). From Qin et al., $2000 \mathrm{~b}$.

changes to $2 \times N$, which can be described as a periodic sequence, in which every $N$ th dimer is missing. The period $N$ of the dimer vacancies decreases with increasing Ge coverage (Teichert et al., 2002), for a coverage of one monolayer, $N \approx 13$. If the Ge coverage exceeds two monolayers, the $2 \times N$ reconstruction further develops, creating a periodic sequence of 2D "patches" $(M \times N$ reconstruction: Voigtländer, 2001; Rastelli, von Kaenel, Albini, et al., 2003). Detailed experimental and theoretical studies of the movement of Si-Ge dimers at a $2 \times 1$ reconstructed $\mathrm{Si}(001)$ surface have shown that an exchange of $\mathrm{Ge}$ adatoms with $\mathrm{Si}$ atoms in the substrate is possible, since the corresponding energy barrier is rather low (Lu et al., 2000; Qin et al., 2000a, 2000b).

Rastelli, von Kaenel, Albini, et al. (2003) studied the behavior of this structure during an overgrowth by a thin Si layer by STM and ab initio molecular-dynamics calculations. The surface structure $M \times N$ evolves back to 2 $\times N$ and eventually $2 \times 1$, which is explained by the diffusion of $\mathrm{Si}$ adatoms through the Ge layer.

For the submonolayer deposition of InAs on As-rich GaAs(001), a change in the surface reconstruction from $2 \times 4$ to $4 \times 2$ or $6 \times 2$ also occurs (Xue et al., 1997; Xue and Sakurai, 1998; Krzyzewski et al., 2002a).

The step-flow growth model describes epitaxial growth as a lateral movement of monolayer steps via attachment of adatoms at the steps and their kinks [see the Burton-Cabrera-Frank model after Burton et al. (1951) and Villain (1991)]. This growth mode prevails if the mean free path for adatom diffusion across the steps is larger than the mean step distance. During step movement, there is also a certain probability of nucleating monolayer islands at the terraces between the steps. If those 2D islands coalesce, a smooth monolayer is formed again, called layer-by-layer growth (Tersoff et al., 1994).

Most theoretical simulations of step-flow growth are based on continuum calculations or an atomistic Monte Carlo method (see Villain, 1991; Rost et al., 1996; and references therein). The former approach is based on a continuum growth equation expressing mass conservation and taking into account a surface diffusion inhomo- 


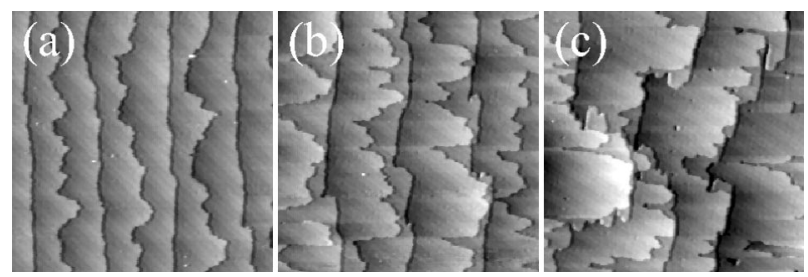

FIG. 3. STM pictures $\left(350 \times 350 \mathrm{~nm}^{2}\right)$ demonstrating the movement of steps during step-flow growth of $\mathrm{Si}(001)$ : panel (a) the starting morphology before growth; panels (b) and (c) steps at Si coverages of 1.2 and 3.5 ML's, respectively. From Voigtländer, 2001, reprinted with permission from Elsevier. Copyright 2001.

geneity due to the local surface curvature and a modification of the adatom flux due to the presence of steps (Burton et al., 1951) Both approaches yield very similar numerical results.

The step-flow growth mode can also be used for the fabrication of one-dimensional $\mathrm{Ge}$ or Si quantum wires (Kawamura et al., 2003). Alternating deposition of $\mathrm{Si}$ and $\mathrm{Ge}$ atoms in the step-flow regime results in the creation of a one-monolayer-thick periodic array of $\mathrm{Si}$ and Ge wires oriented parallel to the surface steps.

$\mathrm{Ge} / \mathrm{Si}(001)$ and $\mathrm{SiGe} / \mathrm{Si}(001)$ systems with $2 \times 1$ or 2 $\times N$ surface reconstructions serve as a model system for step-flow growth. The resulting surface morphology depends substantially on both the starting density of monolayer steps (i.e., the crystallographic miscut of the surface) and on the azimuthal miscut direction. The macroscopic step direction has to be perpendicular to the miscut azimuth. A simple case is a miscut parallel to [110], i.e., steps along the [110] direction. Since the dimers of the $2 \times 1$ reconstructed surface are along the $\langle 110\rangle$ directions, two types of monolayer steps exist, namely, $S_{\mathrm{A}}$ and $S_{\mathrm{B}}$, oriented parallel to the dimer rows on the upper terrace and perpendicular to them. The lateral mobilities of the $S_{\mathrm{A}}$ and $S_{\mathrm{B}}$ steps are different due to the anisotropy of adatom diffusion across the terraces as well as to different sticking coefficients for adatoms at $S_{\mathrm{A}}$ and $S_{\mathrm{B}}$ steps. Adatoms move about 1000 times faster along the dimer rows than across to them. Therefore, on a $T_{\mathrm{A}}$ terrace, the atoms move mainly along the steps, while on a $T_{\mathrm{B}}$ terrace the adatoms move preferably perpendicular to the steps. In addition, the adatoms are much better trapped at the ends of dimer rows on $S_{\mathrm{B}}$ steps than at the sides of dimer rows on $S_{\mathrm{A}}$ steps (Voigtländer, 2001). Consequently $S_{\mathrm{B}}$ steps move much faster than $S_{\mathrm{A}}$ steps and exhibit a different morphology: $S_{\mathrm{A}}$ steps are straight, while $S_{\mathrm{B}}$ steps are randomly undulating [see Fig. 3(a)]. The equilibrium morphology of a vicinal $\mathrm{Si}(001)$ surface consists therefore of a sequence of close $S_{\mathrm{A}}-S_{\mathrm{B}}$ pairs. Depending on temperature and miscut values, the $S_{\mathrm{A}}-S_{\mathrm{B}}$ pairs may also create double steps $D B$ dividing two adjacent $T_{\mathrm{B}}$ terraces (Pehlke and Tersoff, 1990; Poon et al., 1990). However, the final surface coverage by $T_{\mathrm{B}}$ terraces is never $100 \%$ (see Fig. 3), mainly due to the pinning of $S_{\mathrm{A}}$ steps at structural defects. A completely different surface morphology is observed if the azimuthal direction of the miscut differs from $\langle 110\rangle$. As the macroscopic direction of the steps is always perpendicular to the miscut direction, for a general miscut orientation the monolayer steps consist of microscopic $S_{\mathrm{A}}$ and $S_{\mathrm{B}}$ segments. If the miscut direction is close to $\langle 100\rangle$, the resulting step morphology can be described as a zigzag pattern of $S_{\mathrm{A}}, S_{\mathrm{B}}$, and double steps. In a narrow range of growth parameters $\left(480<T_{g}<550^{\circ} \mathrm{C}\right.$ for a growth rate of $\left.0.8 \AA / \mathrm{s}\right)$, growth kinetics leads to a nearly periodic sequence of tiny crests elongated parallel to the miscut direction; for $T_{\mathrm{g}} \simeq 550^{\circ} \mathrm{C}$, these crests merge and form a ripple pattern parallel to the miscut azimuth (Schelling et al., 1999). If the step-flow growth of a $\mathrm{SiGe}$ alloy can be described as a movement of closely spaced $S_{\mathrm{A}}-S_{\mathrm{B}}$ pairs (i.e., as a bilayer step flow), compositional ordering is observed (Jesson, Pennycook, et al., 1993b).

Sutter et al. (2003) investigated in detail by STM the interaction of the dimer vacancy lines with the $S_{\mathrm{A}}$ steps around a $2 \mathrm{D}$ island deposited on a terrace. The authors demonstrated that the dimer vacancy lines are repulsed from the steps. This interaction limits the step-flow growth and causes an increase of the nucleation rate of 2D islands, therefore contributing to the onset of the nucleation of $3 \mathrm{D}$ islands.

\section{Step bunching}

During $\mathrm{Si}$ homoepitaxy as well as in $\mathrm{SiGe} / \mathrm{Si}$ heteroepitaxy, the step-flow growth mode is unstable against the bunching of $S_{\mathrm{A}}, S_{\mathrm{B}}$, or $D B$ steps. In general, this effect can be explained by two different mechanisms (see Duport et al., 1995) namely, by coalescence of steps due to the elastic strain in the growing layer (the strain model), or by purely kinetic phenomena connected with diffusion anisotropy and step-edge detachment of the adatoms (the broken-bond model). The strain model is based on an interplay of effective step repulsion and step attraction during growth (Tersoff et al., 1995). The repulsive interaction between the steps depends on surface energy and arises from the intrinsic stress field of the steps, the force dipole (Marchenko and Parshin, 1980; Marchenko, 1981; Alerhand et al., 1988; Poon et al., 1990). The corresponding interaction energy drops with step distance $L$ as $L^{-2}$ and it is independent of the lattice mismatch. The attractive attraction between two steps results from the elastic stress in the growing epitaxial layer; its interaction energy is proportional to $\sigma^{2} \ln (L)$, where $\sigma$ is the lateral elastic mismatch stress (Tersoff et al., 1995). The effective attractive step interaction can be explained qualitatively by local elastic stress relaxation. Xie et al. (1994) used atomistic simulations to calculate the energy of monoatomic steps on a strained surface. They found that a compressive stress reduces the step free energy, leading to step bunching.

Liu et al. (1998) found in numerical simulations that the strain model of step bunching leads to an increase of the average bunch size with growth time $t$ as $t^{n}$. Here $n$ depends mainly on the adatom flux. The simulations 


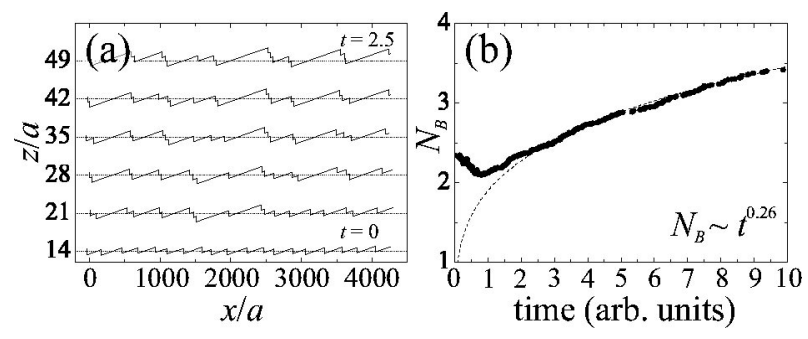

FIG. 4. Evolution of a stepped surface calculated using the strain model for step bunching: (a) time evolution of the average bunch size; (b) growth time expressed in arbitrary units. Adapted from Tersoff et al., 1995.

yield $n \approx 0.25$ for zero flux (see Fig. 4). With increasing flux $n$ decreases, and for larger flux values even a step debunching is predicted by Tersoff et al. (1995). Patriarche et al. (2000) successfully explained the interface morphology of short-period InGaAsP/InP superlattices grown on $\operatorname{InP}(001)$ using the strain model of step bunching. The strain in InGaAsP layers was tuned from compressive to tensile by changing their chemical composition. In compressively strained layers, step bunching at the InGaAsP surface was observed for all miscut values up to several degrees, creating a quasiperiodic sequence of step bunches and flat terraces, while the surfaces of InP spacer layers remained nearly atomically flat. This finding supports the strain model of step bunching, from which a planarization of spacer layers without stress follows. For tensile strained layers, different mechanisms set in, in addition to step bunching.

During deposition of an alloy, decomposition of the alloy can occur, which screens out the inhomogeneous strain and partially suppresses step bunching (Tischler $e t$ al., 1995; Tersoff, 1996; Venezuela et al., 1999).

While the concept of stress-induced attractive stepstep interaction successfully explains step bunching during heteroepitaxy, it fails to explain step bunching during homoepitaxy. In this case, the resulting surface morphology depends sensitively on temperature and on the miscut azimuth. The microscopic Burton-Cabrera-Frank model of step movement was used for kinetic Monte Carlo simulations of step bunching during homoepitaxy by Kandel and Weeks (1995). The step movement could be described as the simultaneous bunching of monolayer steps and debunching of the step bunches. During growth, relatively straight, slow-moving step bunches coexisted with fast-moving, meandering single steps.

Another possible reason for the bunching of $S_{\mathrm{A}}$ and $S_{\mathrm{B}}$ steps or double steps is purely geometric, and can be explained by the energy of broken bonds at the surface (Duport et al., 1995). During growth, a larger terrace between the steps collects a large number of adatoms. If the net diffusion current of these adatoms is oriented downwards, the adatoms prefer to stick to the step "downstairs" and larger terraces tend to grow at the expense of smaller ones. If, on the contrary, the adatoms flow upwards and stick preferably to the upper step of the terrace, a larger terrace will shrink and the surface structure is stabilized. The difference between upward and downward adatom fluxes might be caused by the so-called Ehrlich-Schwoebel barrier, which prevents adatoms from hopping downstairs (Schwoebel and Shipsey, 1966). Therefore this barrier stabilizes the growing surface against bunching. While its existence can be justified by simple physical arguments, an "inverse EhrlichSchwoebel barrier" is purely phenomenological (Chalmers et al., 1993) Several theoretical (Shilkrot and Srolovitz, 1997) and experimental investigations have been devoted to an experimental search for the EhrlichSchwoebel barrier in Si. It is generally believed that this barrier is very small for $S_{\mathrm{A}}$ and $S_{\mathrm{B}}$ steps on $\mathrm{Si}$. As for the double steps $D B$, there is a discrepancy between theoretical predictions of a significant barrier and experimental results indicating that it is rather small. In GaAs, the Ehrlich-Schwoebel barrier is very small as well; Robey (2002) investigated kinetic roughening during plasma etching of GaAs and estimated the height of this barrier to be about $0.05 \mathrm{eV}$. Such a small barrier indeed would have no influence on the growth of InAs islands on GaAs (Kratzer, 2004).

Myslivecek et al. (2002) showed in kinetic Monte Carlo simulations that, on a $2 \times 1$ reconstructed Si surface, step bunching during homoepitaxy could be explained by anisotropic $2 \mathrm{D}$ adatom diffusion and by different adatom sticking coefficients at $S_{\mathrm{A}}$ and $S_{\mathrm{B}}$ steps, rather than by assuming energetic barriers.

Recently, step bunching during SiGe heteroepitaxy on $\mathrm{Si}(001)$ was investigated for several Ge concentrations in the growing layer, various orientations of the Si substrate, and various growth temperatures (see Ronda et al., 2000; Schelling et al., 2001; Mühlberger et al., 2003). As shown in Fig. 5, the resulting bunch morphology depends mainly on growth temperature and is nearly independent of mismatch stress. This finding favors a kinetic step bunching model and contradicts stress-mediated models. For larger miscuts of about $4^{\circ}$ from (001) and strained SiGe layers, the step bunches are not straight and they exhibit a zigzag pattern. In this pattern, the bunch decomposes into a sequence of $\{105\}$ facets; this structure represents a first stage of the growth of $\{105\}$ pyramids (Teichert, 2002, Lichtenberger et al., 2004).

\section{Step meandering}

Beside step bunching, other instability mechanisms can lead to quasiperiodic structures during step-flow growth. Step meandering (Bales-Zangwill instability; Bahr et al., 1990) is caused by the mutual influence of adatom density at a growing terrace and the step morphology. From a surface diffusion equation it follows that a protrusion of an upper terrace increases the local adatom density at the lower terrace and decreases the density at the upper terrace. If the adatom mobilities towards the step are different at upper and lower terraces (as is the case on $T_{\mathrm{A}}$ and $T_{\mathrm{B}}$ terraces), this density inhomogeneity enlarges the protrusion, resulting in a wavy step morphology. Alternatively, for Si homoepitaxy and a miscut azimuth along the $\langle 100\rangle$ direction, such a wavy morphology can be explained by bunching of 

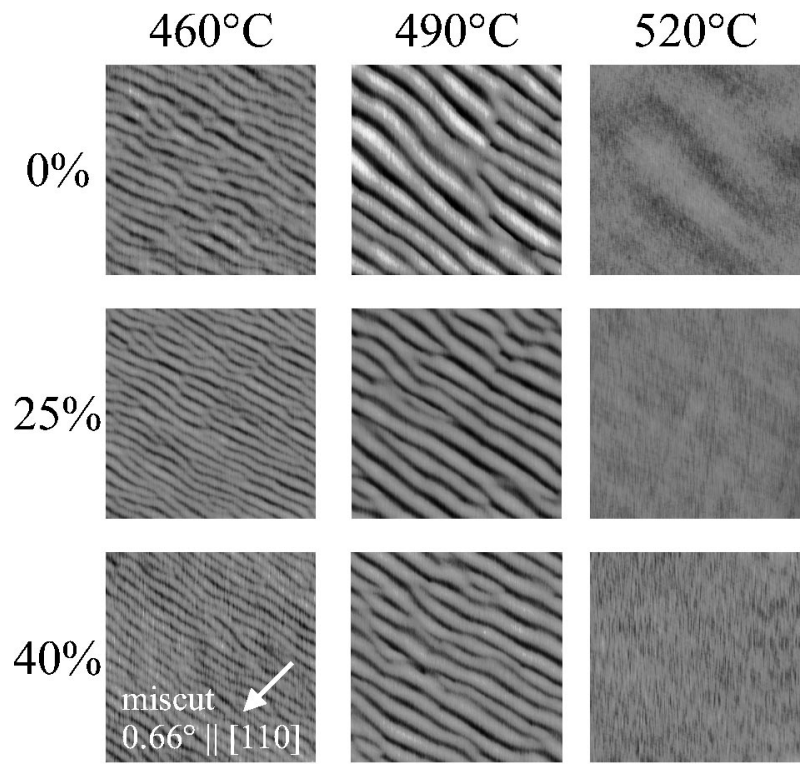

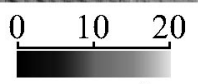

height $(\AA)$

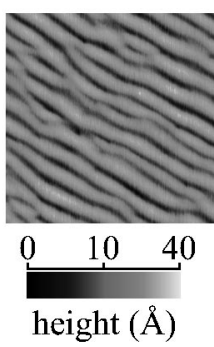

all images: $5 \times 5 \mu \mathrm{m}^{2}$
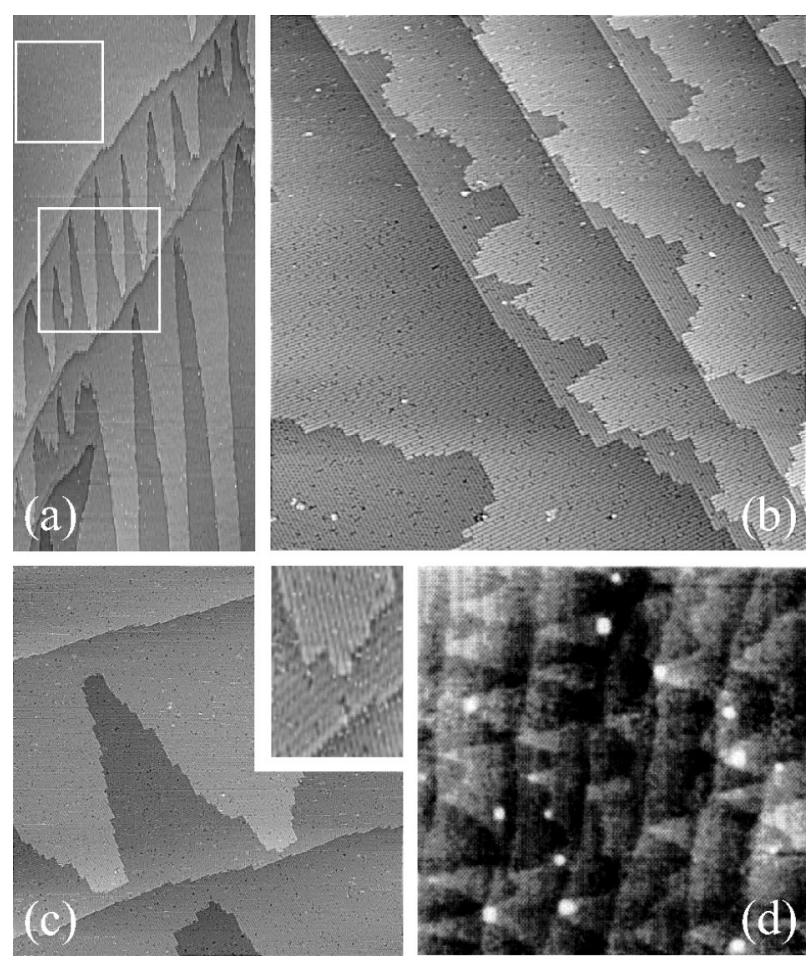

FIG. 6. Si/Ge layers: (a)-(c) STM images at different magnifications of a tensile strained Si layer deposited on a graded SiGe buffer layer. From Jones et al., 1995. (d) AFM image of a $\mathrm{Ge} / \mathrm{Si}$ surface (size $1600 \times 1600 \mathrm{~nm}^{2}$ ) showing a triangular instability of $S_{\mathrm{A}}$ steps caused by the compressive stress in the Ge layer. The white spots are 3D Ge islands nucleated at the apexes of the $S_{\mathrm{A}}$ triangles. From Chen et al., 1995.

modulation of the thicknesses of individual layers. This effect has been observed in III-V superlattices, mainly in InAs/AlAs structures nearly lattice matched to $\operatorname{InP}(001)$ substrates (see Ahrenkiel et al., 1998; Follstaedt et al., 1998, 2000; Twesten et al., 1999).

The modulation amplitude depends on the average strain of the superlattice with respect to the substrate, which can be tuned by changing the relative average thicknesses of the superlattice layers. The modulation was observed only for relatively small strains $|\varepsilon|<0.01$ (Follstaedt et al., 1998). Its direction depends sensitively both on the average strain and on the substrate miscut. For tensile net strain, modulation along [310] and [130] directions is observed, while compressive strain leads to modulations along [100] and [010]. If the substrate has no significant miscut, both modulation directions are present simultaneously, and the resulting structure consists of very short quantum wires with both orientations (Follstaedt et al., 2000).

A theoretical descriptions of the modulation effect can be found in Guyer and Voorhees (1996), Glas (2000), Shilkrot et al. (2000), and Spencer et al. (2001), based on the growth equation in the continuum approximation

$$
\frac{\partial h}{\partial t}=F+\frac{\tilde{D} V_{(\text {at })} \Theta}{k_{B} T} \nabla_{\text {surf }}^{2} \mu_{\text {surf }},
$$

where $h(x, y, t)$ is the height function of the growing surface in time $t$, while $x, y$ are the coordinates along the 
mean surface, $F$ is the incoming adatom flux, $\tilde{D}$ is the surface diffusivity, and $V_{(\text {at) }}$ and $\Theta$ are the atomic volume and atomic surface density. The operator $\nabla_{\text {surf }}$ acts along the surface and $\mu_{\text {surf }}$ is the chemical potential of an atom at the surface, which is affected via curvature by the surface energy density and by the elastic lattice deformation energy close to the surface,

$$
\mu_{\text {surf }}=\mu_{0}+\gamma V_{(\text {at })} \kappa+\hat{\sigma}_{\text {surf }} \hat{S} \hat{\sigma}_{\text {surf }} V_{(\mathrm{at})} / 2 .
$$

Here $\mu_{0}$ is the chemical potential of an atom in nondeformed bulk material, $\gamma$ is the surface tension, $\kappa$ is the surface curvature, $\hat{\sigma}_{\text {surf }}$ is the surface stress tensor, and $\hat{S}$ is the elasticity compliance matrix.

The structure of laterally modulated superlattices has been investigated with plan-view and cross-sectional transmission electron microscopy by Ahrenkiel et al. (1998), Follstaedt et al. (1998, 2000), and Twesten et al. (1999), and with high-resolution x-ray scattering by $\mathrm{Li}$, Holy, et al. (2001, 2002).

\section{Nucleation and growth of two-dimensional islands}

If the mean free path of the adatoms moving at the growing surface is smaller than the mean distance of monolayer steps, 2D islands grow at terraces. In homoepitaxy, the nucleation and growth of $2 \mathrm{D}$ islands is entirely determined by the difference in free energies between a single adatom at the surface and an atom incorporated in an island. From the thermodynamics of nucleation, the critical radius $R_{\text {crit }}$ of an island is expressed as (Shchukin et al., 2003)

$$
R_{\text {crit }}=\frac{a}{2} \frac{w}{w+k_{B} T \ln q},
$$

where $a$ denotes the lattice parameter, $w$ the binding energy per atom, and $q$ the adatom density normalized to unity. For the usual growth temperatures between 300 and $700{ }^{\circ} \mathrm{C}$ and a binding energy of the order of $1 \mathrm{eV}$, a large critical island radius can be obtained only if the adatom density does not exceed the equilibrium value $q_{\text {eq }}=\exp \left[-w /\left(k_{B} T\right)\right]$ by more than two orders of magnitude. After nucleation of islands exceeding the critical size, further growth occurs by incorporating adatoms. The adatom density decreases until $q_{\mathrm{eq}}$ is reached. In the last stage of growth, a ripening process occurs in which the larger islands grow and the smaller ones dissolve.

In heteroepitaxy, the mismatch between the growing layer and substrate substantially affects the nucleation and growth of 2D islands. The growing surface consists of stress domains. Their shape results from the interplay of the surface energy (depending both on surface energy of the adsorbed layer and the bare substrate), the energy of the domain boundaries, and the elastic energy stored in the domain. The simplest structure is realized by parallel stripe domains (Alerhand et al., 1981; Marchenko, 1981); another type of domain is that of circular 2D droplets periodically distributed at the surface (Vanderbilt, 1986). Most studies on 2D islands concern metallic surfaces [see Shchukin et al. (2003) for details]; in semi- conductors, InAs (Shchukin et al., 2001) and CdSe (Strassburg et al., 1998) submonolayer islands have been studied.

\section{B. Stranski-Krastanow growth}

In the previous section we discussed the relief of the elastic energy stored in a growing heteroepitaxial layer by the creation of one-dimensional straight or wavy bunches of monolayer steps or 2D monolayer islands. The efficiency of energy relief is increased in a twodimensional self-organization process creating a wavy surface morphology in both directions. This relief of elastic energy is always accompanied by an increase of the surface energy, which depends sensitively on the formation of various crystallographic facets.

For $\mathrm{SiGe}$ on $\mathrm{Si}$, two types of island structures may be observed, namely, shallow mounds with lateral dimensions over $100 \mathrm{~nm}$ and islands with well-developed crystallographic facets.

\section{Asaro-Tiller-Grinfeld instability}

Shallow mounds appear during heteroepitaxy with low lattice misfit (Sutter and Lagally, 2000; Tersoff et al., 2000; Tromp et al., 2000). Their origin lies in the AsaroTiller-Grinfeld (ATG) morphological instability of a stressed film (Asaro and Tiller, 1972; Grinfeld, 1986; Srolovitz, 1989; Spencer et al., 1993). A growing stressed surface is unstable against perturbations with wavelengths longer than a critical wavelength

$$
\lambda_{\text {crit }}=\frac{1-\nu}{2 G(1+\nu)^{2}} \frac{\pi \gamma}{\varepsilon_{0}^{2}},
$$

where $\nu$ is the Poisson ratio of the material, $G$ is its shear modulus, $\gamma$ is the surface tension, and $\varepsilon_{0}$ is the misfit strain of the growing layer with respect to the substrate.

The numerical solution of the ATG problem based on growth equation (1) in the continuum approximation was achieved by Spencer and Meiron (1994) and Gao (1994). The solution has the form of a periodic cycloidal surface with deep cusps with a period $\lambda_{\text {crit }}$. The local stress below this surface is highly inhomogeneous; below the cusps it can exceed the plasticity limit of the material, which can lead to misfit dislocation formation. The creation of such dislocations in the cusps has been discussed theoretically by Jesson, Pennycook, et al. (1993a) and observed by a combination of AFM and TEM by Jesson et al. (1995). From the microscopic point of view, the ATG instability corresponds to a strain-driven stepbunching process.

A direct method has been suggested by Jesson, Pennycook, et al. (1993a) for the observation of cusps and their development during growth. The method was used for SiGe layers on Si substrates and it consists in the incorporation of a sequence of very thin Ge marker layers placed at different depths in the SiGe layer. The form of the marker layers was visualized by Z-contrast cross-sectional transmission electron microscopy. The 

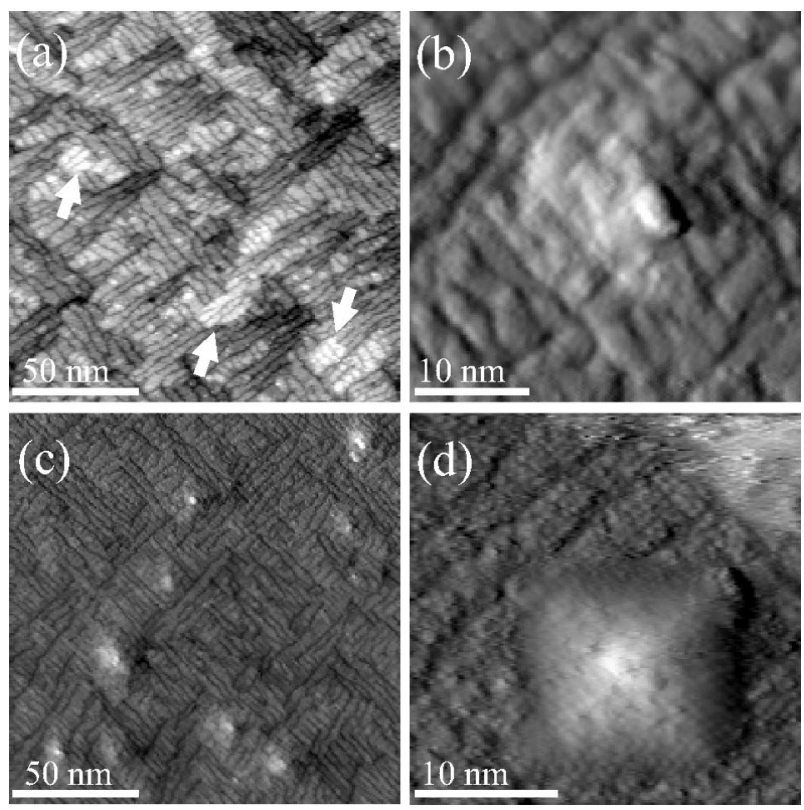

FIG. 7. STM images of the surface evolution during growth of a Ge layer on $\mathrm{Si}(001)$. In panels (a)-(d), the Ge coverage grows from 2.8 to 4.0 ML's. During growth (a)-(c) first mounds (prepyramids) develop, then these convert (d) to $\{105\}$ facetted pyramids. From Vailionis et al., 2000.

experimentally established form of the marker layers (corresponding to the instantaneous profile of the growing SiGe surface) agrees well with the numerical solution of Eq. (1).

During SiGe growth on $\mathrm{Si}(001)$, mounds evolve due to the ATG instability, and the slope of their side walls increases gradually until an angle of about $11^{\circ}$ is reached (see Jesson et al., 2000; Sutter and Lagally, 2000; Tromp et al., 2000; Vailionis et al., 2000), which corresponds to $\{105\}$ facets. Beyond this thickness, the facet angle remains unchanged and $\{105\}$ facetted pyramids develop. The gradual mound evolution and transition to pyramids is documented in Fig. 7. Tersoff et al. (2002) investigated the creation of shallow mounds and their shape transformation by a numerical simulation in the continuum approximation. Tersoff et al. (2002) assumed that the surface energy density $\gamma$ exhibits a minimum for the $\{100\}$ surface orientation, but no deep cusp in the polar diagram $\gamma(\theta)$, i.e., $\{100\}$ is not a true facet. Under this assumption Eqs. (1) and (2) were solved, applying the numerical strain calculation method of Spencer and Meiron (1994). For a certain range of island volumes, two metastable shapes were found, namely, facetless prepyramids and facetted pyramids. This is shown in Fig. 8, where the shapes of SiGe island cross sections are plotted for various volumes along with the size dependence of the island energy. The growing prepyramids remain stable up to the volume $V_{2}$. At this size, a firstorder shape transition takes place and facetted pyramids represent the stable shape. There is an activation energy for this shape transition, so that the prepyramids may grow up to the volume $V_{3}$. The energy barrier between the metastable nonfacetted and the stable facetted shape
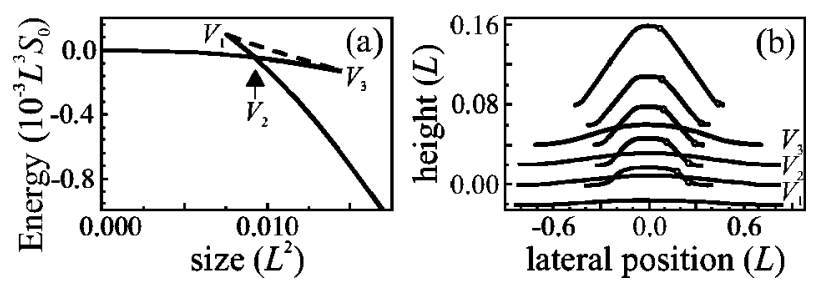

FIG. 8. Dependence of the island energy on volume (a) and the corresponding cross sections of SiGe islands calculated for various island volumes (b). For volumes between $V_{1}$ and $V_{3}$ two (meta)stable shapes exist - a shallow prepyramid without facets and a facetted pyramid. The pairs of solid circles in (b) denote the side facet. From Tersoff et al., 2002.

is represented by the distance between the solid line and the dotted line (for $V>V_{2}$ ). Rastelli and von Känel (2003) and Rastelli, von Känel, Spencer, and Tersoff (2003) investigated by STM the transition of prepyramids to facetted $\{105\}$ pyramids via an intermediate shape, namely, $T$ pyramids $(\{015\}$ pyramids with a rounded top). These $T$ pyramids exhibit the onset of $\{105\}$ facets. The experimental findings agree well with theoretical considerations by Tersoff et al. (2002).

The barrierless formation of prepyramids in the case of low misfit also follows from a microscopic kinetic model (Kaganer and Ploog, 2001) based on the BurtonCabrera-Frank model of monolayer step movement. This model, however, does not include the shape transition from the unfacetted mounds to facetted pyramids and domes for higher island volumes.

For the deposition of $\mathrm{SiGe}$ alloys containing less than $20 \% \mathrm{Ge}$ and at temperatures below $600^{\circ} \mathrm{C}$, Koch et al. (2001) observed a growth mode in which no wetting layer formed, but very small 3D islands $(4-6 \mathrm{~nm})$ were formed directly.

\section{Island nucleation}

Island growth via the Asaro-Tiller-Grinfeld instability occurs only for small misfits and does not exhibit any energetic barrier for island growth. For larger misfit values, a different process sets in, namely, the nucleation of 3D islands on a smooth surface of the wetting layer, termed the Stranski-Krastanow growth mode (Stranski and Krastanow, 1938; Eaglesham and Cerullo, 1990; Mo et al., 1990). In contrast to the ATG process, island nucleation in the Stranski-Krastanow growth mode has a certain activation energy that must be overcome. This follows from simple energetic considerations (see, for instance, Tersoff and Le Goues, 1994; Brunner, 2002). The creation of an island with volume $V$ having facets inclined by an angle $\theta$ with respect to the surface requires an energy

$$
\Delta E=4 \Gamma V^{2 / 3} \tan ^{1 / 3} \theta-6 A V \tan \theta,
$$

where $\Gamma=\gamma_{e} / \sin \theta-\gamma_{s} \cot \theta$, and $\gamma_{s}$ and $\gamma_{e}$ are the surface free energy per unit area of the facet oriented parallel to the mean surface and inclined by $\theta$, respectively; $A$ $=\sigma_{\|}^{2}(1-\nu) /(2 \pi G)$, and $\sigma_{\|}$is the in-plane misfit stress in 


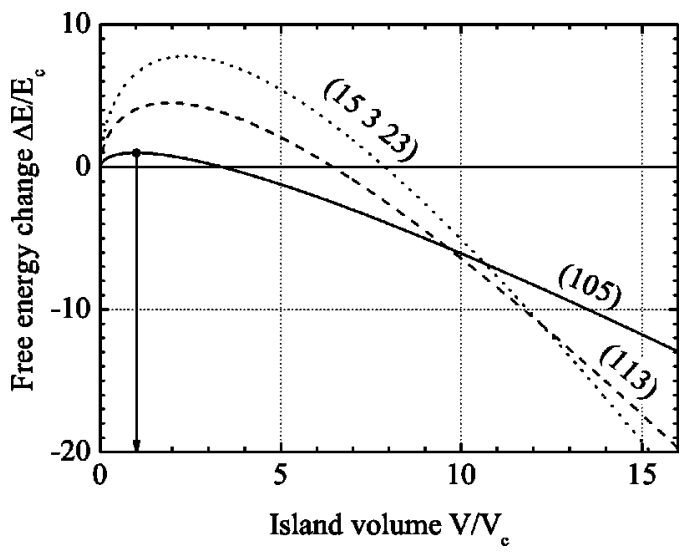

FIG. 9. The volume dependence of the energy necessary to create a pyramidal island, calculated for three different orientations of the facets. Energy and volume are normalized to the critical values of $\{105\}$ facetted pyramids. Adapted from Tersoff and LeGoues, 1994; Brunner et al., 2000.

the growing layer. Figure 9 shows how this energy depends on island volume. Below a critical volume $V_{c}$, the energy of the island increases with volume up to a maximum energy $E_{c}$. This energy represents an activation barrier for island nucleation. In an in situ STM study during molecular-beam epitaxy at $300^{\circ} \mathrm{C}$ by Jesson et al. (2000), the temporary existence of sub critical nuclei near pits in the surface was confirmed. If the nucleus size exceeded $V_{c} \approx 1500$ atoms, the energy of the island decreased with volume due to the second term in Eq. (5). This behavior corresponds to an Ostwald ripening process, where larger islands grow at the cost of smaller ones.

Budiman and Ruda (2000) presented a detailed theoretical description of island nucleation, based on the calculation of the free energy of an island and the Landau theory of phase transitions. The nucleation is also affected by the elemental segregation in the originally flat wetting layer. Cullis et al. (2002) demonstrated this effect in InGaAs/GaAs systems by atomistic simulations. They showed that the concentration of In atoms was highly enhanced in the uppermost monolayer, from nominally $25 \%$ up to $80 \%$. This enhancement increases the local misfit stress and makes it possible to overcome the energetic nucleation barrier. Furthermore, both In and $\mathrm{Ge}$ segregation influence island nucleation (Brunner, 2002; Cullis et al., 2002).

The essence of Stranski-Krastanow growth lies in the transition from planar growth of the wetting layer to 3D island growth. The continuum growth equation (1) with the chemical potential of the adatoms in Eq. (2) cannot account for this transition, since it predicts an onset of undulation already at the very beginning of deposition. In order to explain planar growth for low coverages, Eisenberg and Kandel (2002) considered the elastic anisotropy of the growing layer. This leads to a dependence of the elastic energy density, which influences the chemical potential in Eq. (2), as well as the total thickness of the deposited layer, suppressing the onset of the
ATG instability for thicknesses below a critical value $h_{c}$. A numerical analysis reveals that $h_{c}$ decreases with increasing mismatch and for a mismatch of $4 \%(\mathrm{Ge}$ on $\mathrm{Si}$ ) equals approximately four monolayers.

The continuum description of Stranski-Krastanow growth based on Eqs. (1) and (2) can give only qualitative results, since the microscopic nature of adatom diffusion and incorporation is not accounted for. This can be done only by atomistic calculations, using for instance kinetic Monte Carlo simulations based on interaction potentials obtained by $a b$ initio calculations. For InAs/GaAs, this method was employed by Penev et al. (2001) and Kratzer et al. (2002). The Ge/Si system was investigated by Montalenti (2003). The simulations yielded the influence of surface strain both on the heights of the energetic hopping barriers and on the depths of the binding energy minima between the barriers.

For $\mathrm{SiGe}$ islands grown on $\mathrm{Si}$, the most important parameter controlling island nucleation is the Ge content in the wetting layer (Sullivan et al., 1999). Around each island, the local strain creates a Ge-depleted zone, where the nucleation of another island is less favorable. Hence the density of the resulting SiGe islands is inversely proportional to the $\mathrm{Ge}$ content in the wetting layer. Capping of a thin Ge wetting layer by a Si layer at higher temperatures decreases the critical thickness and leads to island nucleation even if the amount of $\mathrm{Ge}$ alone is below the critical thickness for StranskiKrastanow growth (Usami et al., 2000b). While island nucleation is generally a statistical process, monoatomic steps or step bunches on a vicinal surface represent preferred nucleation sites (Leon et al., 1997).

\section{Island shapes}

Growth and postgrowth annealing studies have shown that Stranski-Krastanow-grown islands are usually stable against ripening. Under given growth conditions they have well-defined sizes and shapes. In $\mathrm{SiGe} / \mathrm{Si}$, essentially four forms of islands are observed: shallow mounds (prepyramids), square pyramids with $\{105\}$ facets, "hut clusters"-elongated pyramids with $\{105\}$ facets-and large domes with facets in several orientations (Eaglesham and Cerullo, 1990; Mo et al., 1990; Medeiros-Ribeiro, Bratkowski, et al., 1998) Figure 10 shows the size distribution of these island types as well as their evolution during growth (inset), determined by AFM and STM during Ge deposition on $\mathrm{Si}(001)$. In the first stage of growth, shallow prepyramids appear that later convert via $T$ pyramids to pyramids. Large domes form for $\mathrm{Ge}$ coverages above five monolayers, finally plastically relaxed superdomes are formed with misfit dislocations at their bases. Pyramids and domes are observed during growth at higher temperatures, while the much smaller hut clusters form at lower growth temperatures.

The facet structure of self-assembled islands has been studied by in situ STM with atomic resolution. A $\{105\}$ facet consists of (100) terraces with steps along the $\langle 100\rangle$ 


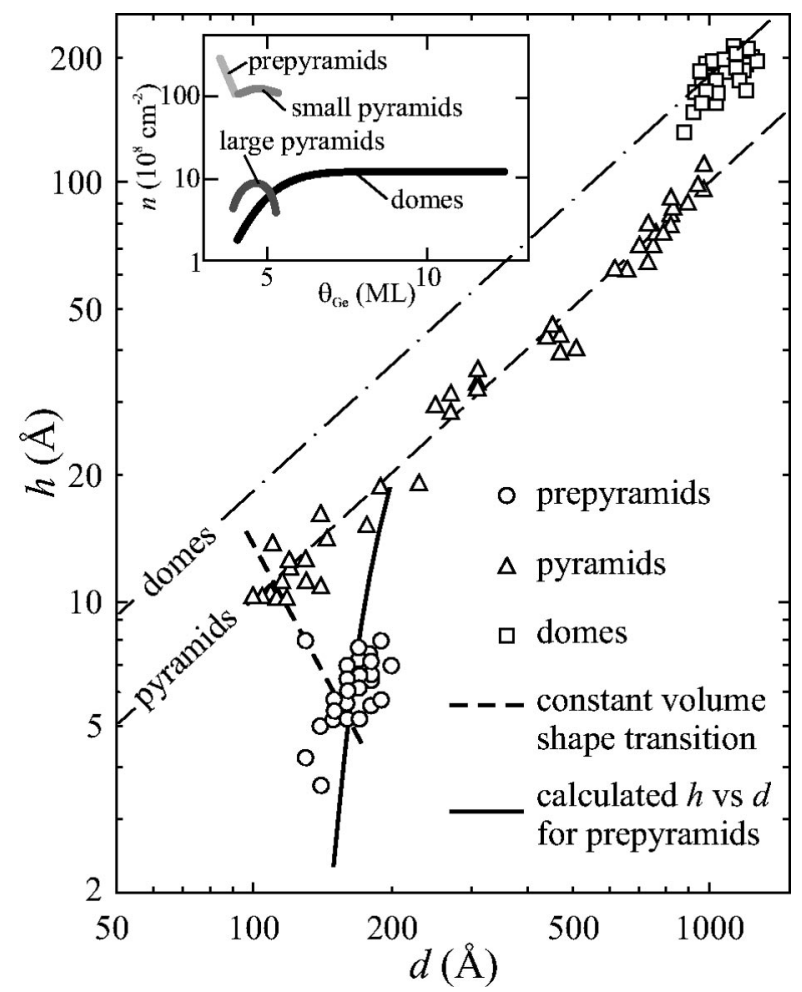

FIG. 10. The size distribution of several island types occurring during deposition of $\mathrm{Ge}$ on $\mathrm{Si}(001)$. The inset shows the time evolution of the island density for different types. Adapted from Vailionis et al., 2000.

direction and a terrace width of only two atoms. The dangling bonds on the terrace create $\langle 110\rangle$ dimers, which are consequently oriented $45^{\circ}$ with respect to the steps (Raiteri et al., 2002). A detailed study of facet growth based on an atomistic simulation and in situ STM by Kastner and Voigtländer (1999) showed that there is an energetic barrier for the nucleation of every atomic $\{105\}$ layer. Although a square shape corresponds to thermodynamic equilibrium, kinetic growth limits lead to elongated huts for growth temperatures lower than about $530{ }^{\circ} \mathrm{C}$. The observed island shapes are explained by a nucleation barrier for steps on completed facets which becomes higher for larger facets. This leads to elongated islands, as islands grow faster in the direction of a small facet than of a large facet. The same model explains the observed deceleration of island growth with island size, stabilizing the pyramid sizes and narrowing the island size distribution. The model predicts the growth of the $\{105\}$ facet in the layer-by-layer regime, with an energetic barrier for the nucleation of each $\{105\}$ monoatomic layer. The layer growth proceeds from the bottom of the island to the apex.

Kinetic Monte Carlo simulations of the growth of reconstructed $\{105\}$ terraces have demonstrated a pronounced strain dependence. In contrast to the findings of Kastner and Voigtländer (1999), if the pyramid apex is largely elastically relaxed, monolayer steps move from the apex towards the pyramid base in a step-flow growth mode (Montalenti, 2003). This was confirmed recently
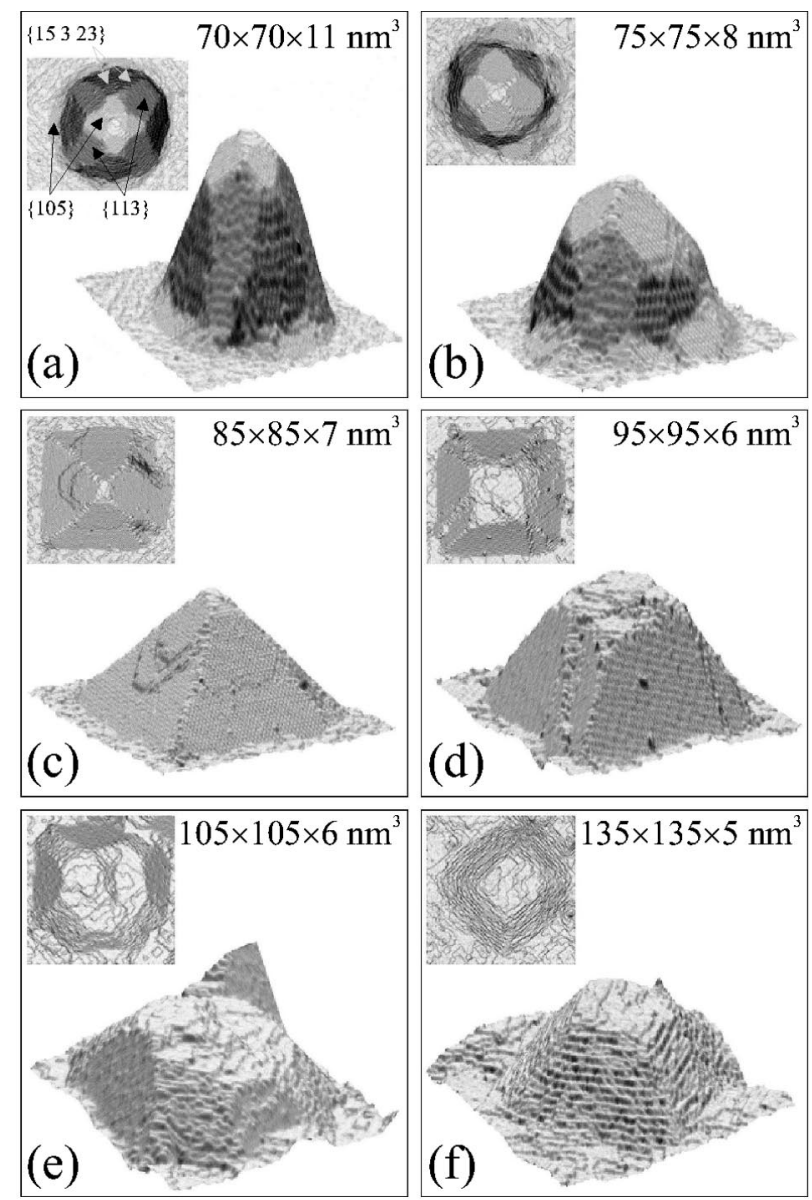

FIG. 11. Typical Ge island shapes obtained by STM during Si capping of Ge domes grown on $\mathrm{Si}(001)$ : (a),(b), domes; (c) pyramids; (d)-(f) prepyramids. The Si coverages are 0, 1, 2, 4, 8, and 16 ML's for panels (a)-(f). From Rastelli et al., 2001.

by STM observations of the transition from pyramids to domes by Rastelli (1999), who observed a top-to-bottom movement of the $\{105\}$ steps and their bunching, leading to a steepening of the facet. This attachment of the adatoms from the island apex leads finally to the pyramidto-dome transition. A similar conclusion was reported by Johansson and Seifert (2002).

The structure of Ge domes on $\mathrm{Si}$ is more complicated. In addition to the $\{113\}$ facets following from the energy considerations (Fig. 9), other crystallographic facets are observed, namely $\{105\},\{15323\}$, and a top (001) facet parallel to the substrate surface (Rastelli et al., 2001; Rastelli, Kummer, and von Kaenel, 2002; Rastelli, Mueller, and von Kaenel, 2002). In Fig. 11, typical shapes of (a),(b) Ge domes, (c) pyramids, and (d),(e),(f) shallow mounds (prepyramids) are presented, obtained by STM with atomic resolution. This sequence of shapes was obtained during Si overgrowth (Rastelli, Kummer, and von Kaenel, 2002).

The shape of the superdomes was investigated by STM by Rastelli and von Känel (2002), who observed in addition to the $\{113\}$ and $\{15323\}$ also $\{111\},\{126\}$ facets and steeper $\left\{\begin{array}{lll}4 & 20 & 23\end{array}\right\}$ ones. The top $\{001\}$ facet of a superdome exhibits the $2 \times 1$ reconstruction, indicating the 

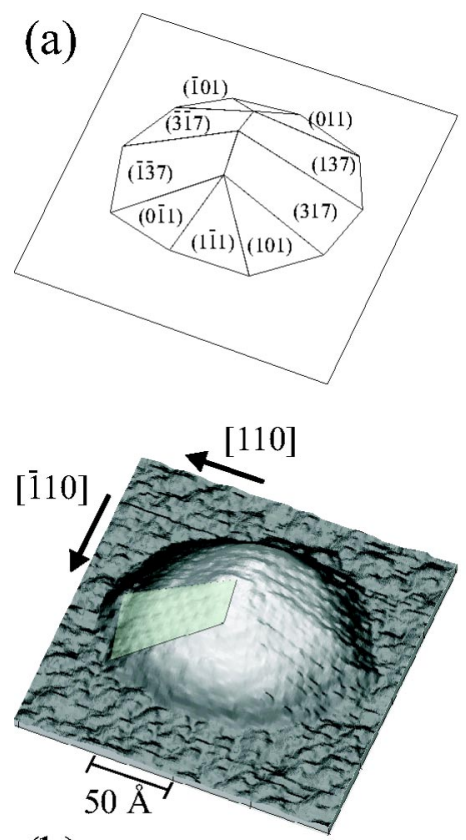

(b)
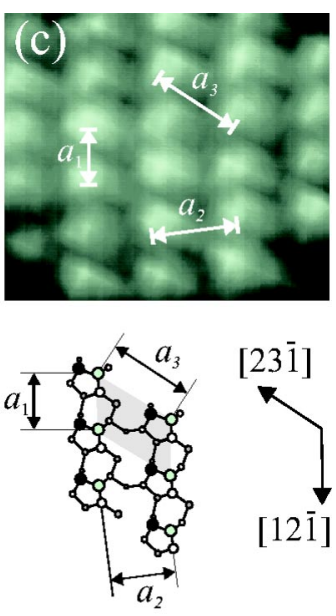

$a_{1}=6,5 \pm 0,1 \AA$

$a_{2}=9,0 \pm 0,3 \AA$

$a_{3}=10,5 \pm 0,2 \AA$

$\measuredangle\left(a_{1}, a_{2}\right)=82,4^{\circ} \pm 0,7^{\circ}$

(d)

FIG. 12. (Color in online edition) Facetting of InAs islands (a) sketch of facets of an InAs islands on $\mathrm{GaAs}(001)$ obtained by STM; (b) and (c) zoom of the reconstructed $\{\overline{1} \overline{3} 7\}$ facet; (d) structure model of facet reconstruction. From Marquez et al., 2003.

full relaxation of the superdome lattice. Remarkably, the dislocated superdomes grew almost vertically, except for a sudden periodic lateral dilatation each time a new misfit dislocation was formed (LeGoues et al., 1994).

The equilibrium shape of InAs islands on $\mathrm{GaAs}(001)$ has been investigated theoretically by Moll et al. (1998) who calculated the total island energy due to strain, surface, and edge contributions. The islands were $\{110\}$ pyramids or $\{110\}\{111\}(001)$ hut clusters, depending on island volume and the reconstruction type of the GaAs(001) surface. An in situ STM study by Marquez et al. (2001) showed InAs islands with $\{137\}$ facets (see Fig. 12). These facets are thermodynamically stable only up to a certain island volume. For larger volumes, the shape evolves into islands with steeper $\{101\}$ and finally $\{111\}$ facets, in analogy to the pyramid-to-dome transition in the Ge/Si system (Rastelli, 2004).

During postgrowth annealing, pyramids and domes are stable, whereas hut clusters disappear (MedeirosRibeiro, Kamins, et al., 1998; Kamins et al., 1999). In several works, however, a ripening process was reported, in which small pyramidal islands grew and transformed into domes and the larger domes partially dissolved and adopted pyramidal shapes. The coarsening process during growth is affected by the elastic interaction between neighboring islands. This interaction leads to an increase of the mean island size, which is faster than in the case of Ostwald ripening (Floro et al., 2000). In addition to the Ostwald ripening mechanism, based on adatom diffusion between the islands, another ripening process may occur caused by the coalescence of touching islands.
This process, however, is suppressed due to the elastic strains in favor of Ostwald ripening, which affects the positions and the island size distribution of densely packed island arrays (Jesson et al., 2004).

The transition from pyramids to domes during growth leads to a bimodal island size distribution (see, for example, Ross et al., 1998, 1999). The pyramid-to-dome transition is of thermodynamic nature and represents a first-order phase transition (Medeiros-Ribeiro, Bratkowski, et al., 1998; Liu, Gibson, et al., 2000), as was demonstrated by postgrowth annealing of domes. While in the first stage of annealing an intermixing between dome and wetting layer takes place, reducing the strain energy, in the second stage the domes evolve back into pyramids (Henstrom et al., 2000).

Island coarsening happens only in a specific temperature window. Outside this window, the islands are relatively stable due to the suppression of strain energy relaxation by strong surface-energy anisotropy and strong film-substrate interaction (Shchukin et al., 1995; Chiu, 1999; Shchukin and Bimberg, 1999).

The thermodynamics of the formation and transformation of the different island families was studied by Daruka and Barabasi (1997, 1998), Daruka, Tersoff, and Barabasi (1999), and Daruka and Tersoff (2002). A dependence of the equilibrium shape on coverage and misfit stress was found in the form of a phase diagram. Equation (5) describing the energy of a facetted island has been improved by Rudd et al. (2003) who also included the energy of the island edges and island-island interaction. Rudd et al. simulated the equilibrium distribution of island shapes and sizes. They established a phase diagram of the equilibrium relative population of pyramids and domes as a function of Ge coverage on $\mathrm{Si}(001)$ and of the growth temperature. At low temperatures (down to $450{ }^{\circ} \mathrm{C}$ ) and low coverages of about 2 ML's, the relative number of pyramids reached $96 \%$, while for larger growth temperatures and larger coverages (up to $15 \mathrm{ML}$ 's) it decreased down to a few percent. The equilibrium simulations were confirmed by AFM. Figure 13 shows two simulation examples for different Ge coverages. The increase in the number of domes at the cost of pyramids is clearly visible.

Vandervelde et al. (2003) observed a completely different shape of SiGe islands: under kinetically controlled conditions of molecular-beam epitaxial growth (growth temperature of $550{ }^{\circ} \mathrm{C}$, growth rate $1 \AA / \mathrm{s}$ ), a sequence of shapes appeared as Ge content was increased, starting from square pits in the wetting layer. For larger Ge content, four small hut clusters were created at the edges of a pit. If the Ge content was further increased, the hut clusters elongated creating a square "quantum fortress" structure with a central depression.

\section{Interdiffusion and segregation}

With increasing growth temperature, Si-Ge intermixing becomes more important. This alloying effect increases the critical size $V_{c}$ of island nucleation (Liao et al., 2000; Capellini et al., 2001). Chaparro et al. (1999) 


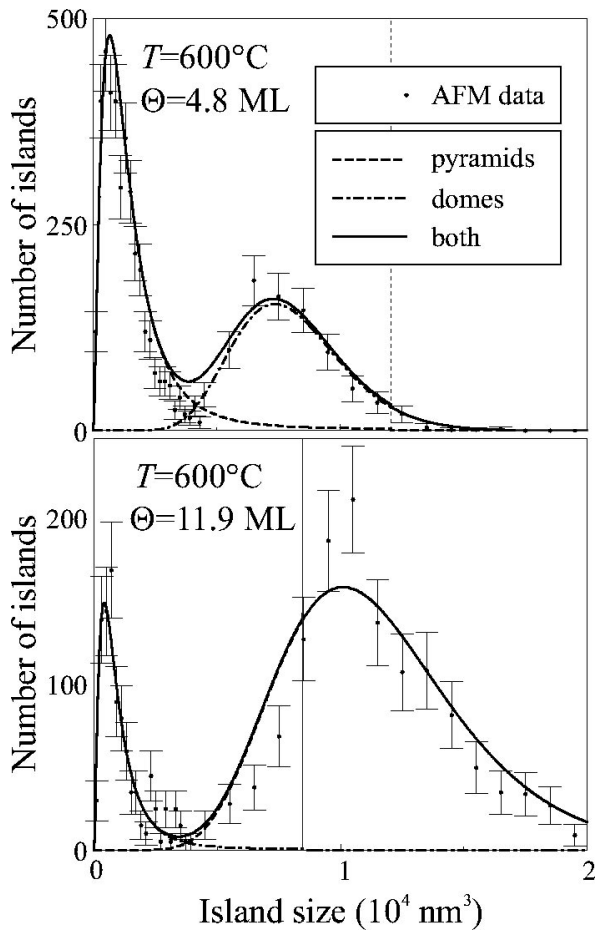

FIG. 13. Size distributions of domes and pyramids calculated by means of equilibrium statistics (lines) for two different Ge coverages $\Theta$, and obtained from AFM (black dots). From Rudd et al., 2003, reproduced courtesy of University of California, Lawrence Livermore National Laboratory, and the U.S. Department of Energy.

and Sonnet and Kelires (2002) found with an atomistic elastic model that the intermixing is driven by strain energy enhancement near the island perimeter, where the strain has a maximum. An effective strain relief mechanism is based on the diffusion from this region towards the island apex. This results in the creation of trenches in the wetting layer around pyramid-and dome-shaped islands (Liao et al., 1999; Chaparro et al., 2000a, 2000b) Rastelli and von Känel (2000) observed this trench creation already in the pyramid phase of growth and postulated that the shallow mounds at the island base transformed into trenches. With increasing island size, these trenches follow the expansion of the island base and move outwards.

Similar phenomena have also been observed for the deposition of SiGe alloys on Si by Floro et al. (1997) and in InAs/InP by Yoon et al. (1999). Recent experimental results by Denker et al. (2003a) indicate that strain energy relief is not the only driving force for Si-Ge intermixing in Ge pyramids. Ge-selective etching reveals that the island corners are highly intermixed, whereas the edges, the centers, and the apexes of the pyramids remain Ge rich. The observed Si enrichment of the corners can be reproduced by numerical simulations based on surface diffusion, whereas volume interdiffusion turns out to be rather insignificant at $T_{g}$ $=550{ }^{\circ} \mathrm{C}$. During postgrowth annealing, higher temperatures give rise to $\mathrm{Si}-\mathrm{Ge}$ intermixing of substrate and is- land atoms that stabilizes dome-shaped islands (Kamins et al., 1999).

The opposite of intermixing, atomic segregation leading to an In enrichment of the island apex, has been observed in InGaAs alloy growth by TEM (Walther et $a l ., 2001)$. Segregation has been studied theoretically by Tersoff (1998), who calculated the free mixing energy of an island. From these calculations, self-capping of a growing InGaAs alloy island creates an In-depleted layer at the island surface, and, in contrast to the results of Walther et al. (2001) an In-rich island core is located close to the island base.

Tersoff (2001) investigated the atomic segregation during the smoothing of a surface ripple, theoretically solving the surface transport equation for each element constituting an alloy. Kinetic surface segregation, caused by the difference in the diffusivity of the alloy components, also affects the rate of the evolution of the surface morphology.

\section{Scaling of the island sizes}

For a submonolayer 2D growth, the size scaling law for $2 \mathrm{D}$ islands can be derived from nucleation theory (Amar and Family, 1995). The size distribution of 2D islands obeys the scaling formula

$$
N(s)=\frac{\Theta}{\langle s\rangle^{2}} f\left(\frac{s}{\langle s\rangle}\right),
$$

where $s$ is the number of atoms constituting an island, $N(s)$ is the number of islands containing $s$ atoms, $\Theta$ is the coverage, and $f$ is a general scaling function depending on the critical number of atoms constituting a stable island. This scaling behavior was confirmed for submonolayer growth of InAs on GaAs (Bell et al., 2000; Krzyzewski et al., 2001, 2002a) and it is similar to the scaling of monolayer islands in homoepitaxy.

Ebiko et al. (1998) also investigated the scaling of the island sizes for the 3D case and found a scaling function similar to the one for homoepitaxy. Thus the misfit strain is irrelevant for the island size distribution. On the other hand, Krzyzewski et al. (2002b) reported no uniform scaling behavior of island sizes during the nucleation and evolution of InAs islands. Consequently it was claimed that the strain has a significant influence for nucleation but is unimportant in later stages.

\section{Dislocations}

A competing strain relief mechanism is based on the creation of misfit dislocations at the substrate surface without any roughening of the wetting layer. The critical nucleus size for Stranski-Krastanow growth scales with the lattice misfit $\varepsilon_{0}$ as $\varepsilon_{0}^{-2}$, while the critical size for plastic relaxation via misfit dislocations scales as $\varepsilon_{0}^{-1}$. Therefore the unfavorable plastic relaxation occurs only below a certain stress value (Tersoff and LeGoues, 1994; Tillmann and Foerster, 2000; Seta et al., 2002). Hence the ATG instability and the insertion of misfit dislocations are competing strain relaxation mechanisms for low $\varepsilon_{0}$. 


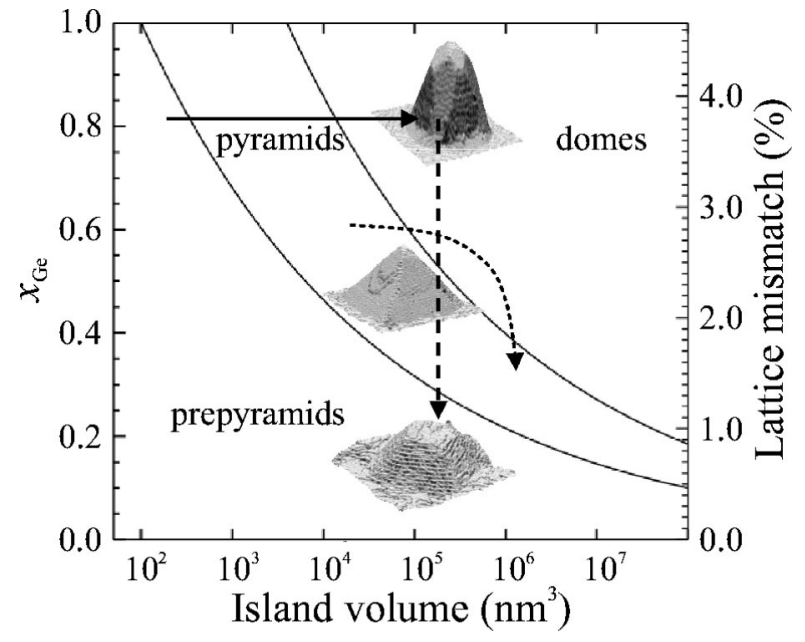

FIG. 14. Shape transition of Ge or SiGe islands grown on $\mathrm{Si}(001)$ during growth (solid arrow), postgrowth annealing (dotted arrow), and Si capping (dashed arrow). The solid curves represent the critical volumes for pyramids and domes. From Rastelli, Kummer, and von Kaenel, 2002, reprinted with permission from Elsevier. Copyright 2002.

The resulting layer morphology depends on growth kinetics; at lower temperatures the surface diffusion is suppressed and the creation of misfit dislocations is more probable (Tersoff and LeGoues, 1994). The equilibrium dislocation configuration within an island has been studied theoretically and experimentally by Spencer and Tersoff $(2000,2001)$ and Ovidko (2002). Most dislocations are created at the island-layer interface, and a tendency towards segregation of dislocations with different Burgers vectors is reported.

\section{Changes of shape, strain, and composition during overgrowth}

An important problem connected with the growth of self-assembled islands is the change of their shape and chemical composition during overgrowth by a cap layer. Changes of an island's strain state due to a cap layer, as well as interdiffusion of island and cap layers (Brunner, 2002), trigger shape changes.

In $\mathrm{SiGe}$ on $\mathrm{Si}(001)$, the overgrowth of $\mathrm{Ge}$ domes with $\mathrm{Si}$ causes a sequence of shape transitions nearly the reverse of those during island growth. During overgrowth, the Ge content in the islands gradually decreases and the $\mathrm{Ge}$ domes convert to $\{105\}$ facetted pyramids and finally to the shallow mounds mentioned above as the very first step of island growth (Usami et al., 2000b; Rastelli et al., 2001; Rastelli, Kummer, and von Kaenel, 2002). The sequence of island shapes is shown schematically in Fig. 14. Note that the volume of the islands increases during capping, hence no very small hut clusters can be found, but rather prepyramids as in island growth for low misfit. The explanation of the shape transitions is similar to that for growth: the equilibrium shape depends on the average strain, which is a function of the island composition and volume. While during growth the strain increases as the island volume is increasing at (more or less) constant composition, during capping the composition drops due to intermixing, while the volume still grows, resulting in a strain reduction and finally a reversal of the shape sequence as compared to island growth. During the transition from $\{105\}$ pyramids to mounds, first a top (001) facet appears. This process can be explained by the embedding of $\mathrm{Si}$ atoms in the islands, a detachment of $\mathrm{Ge}$ atoms from the island apex, and their transport to the side facets, so that their orientation is preserved (see Sutter and Lagally, 1998). When intermixing during overgrowth is suppressed, for instance by applying a low growth temperature (typically below $450{ }^{\circ} \mathrm{C}$ ), the shape of the islands is also preserved (Schmidt, Denker, et al., 2000; Rastelli, Mueller, and von Kaenel, 2002; Stangl, Hesse, et al., 2003). However, the capping layer causes additional strain in the islands (Hesse et al., 2002). If this low-temperature capping is followed by $\mathrm{Si}$ growth at higher temperatures, a smooth Si surface can be recovered (Sullivan et al., 1999; Rastelli, Mueller, and von Kaenel, 2002). A very similar behavior was found for InAs islands covered by GaAs or Ga-rich InGaAs capping layers (Lian et al., 1998; Saito et al., 1998). If InAs islands are covered by a Ga-rich InGaAs overlayer, the inhomogeneous strain around the islands causes a partial decomposition of this layer, so that the effective size of the island increases (Guffarth et al., 2001).The intermixing between the islands and their neighborhood during growth (Wiebach et al., 2000; Kegel et al., 2001; Schülli et al., 2003) and overgrowth has been investigated using ex situ high-resolution x-ray diffraction (Hesse et al., 2002), determining the vertical composition profile of uncapped and buried islands. Obviously, nominally pure $\mathrm{Ge}$ domes on $\mathrm{Si}(001)$ are intermixed already after growth, with a Ge content increasing from island base to apex. Depending on growth temperature, the maximum Ge concentration can reach $70 \%$ to $100 \%$, while the Ge content at the island's base is usually around $30-50 \%$. During overgrowth at temperatures above $600^{\circ} \mathrm{C}$, the maximum value is drastically decreased down to about $40 \%$. If domes are covered by a low-Ge concentration layer, their shape and a high Ge content can be preserved (Mateeva et al., 1999).

\section{Correlation of quantum dot positions}

An important parameter affecting the optical performance of self-organized semiconductor islands is the homogeneity of their size and shape. The size of a growing island is affected by the area from which the island is "collecting" adatoms. Therefore island homogeneity is always improved if the islands are periodically distributed at the growth surface.

In general, the nucleation positions of islands are affected via the chemical potential for adatoms by two factors: (i) the morphology of the growing surface and (ii) inhomogeneous surface stress originating from islands buried below the surface or from neighboring islands at the same surface. 
The wavy morphology of a step-bunched surface can act as a template, inducing a nearly periodic island arrangement (Leon et al., 1997; Sakamoto et al., 1998; Brunner et al., 2000). This enables the mean island distance to be tuned via the substrate miscut. However, the periodic arrangement can be achieved mainly across the bunches, and hardly at all in the direction along the bunches.

\section{Lateral island correlation}

The mutual influence of islands at the same surface is rather weak and can be observed experimentally only for growth at very small rates and close to thermodynamic equilibrium, for instance in liquid-phase epitaxy (Dorsch et al., 1998). Using kinetic Monte-Carlo simulation, Meixner et al. (2001) systematically studied the lateral arrangement of $\mathrm{SiGe}$ islands on $\mathrm{Si}(001)$. The results agree well with AFM observations showing that the islands are arranged in chains parallel to the elastically soft 〈100〉 directions (Holý, Stangl, et al., 1999).

The interaction between 2D islands at the same surface can affect both the size distribution and the periodicity of the island positions. The former is usually described as island coarsening, while the latter can be achieved only if the interaction is strong enough to induce the migration of whole islands, as has been shown theoretically by Liu, Li, and Lagally (2001).

\section{Vertical island correlation}

In a multilayer, the local surface stress caused by islands buried at various interfaces accumulates and may result in the arrangement of islands in vertical columns, observed in InAs/GaAs by Xie et al. (1995). In general, the critical thickness of the Stranski-Krastnow growth in the second layer is reduced compared to the first one, and this difference is ascribed to the influence of the local strain fields propagated from buried islands (Schmidt and Eberl, 2000; Dunbar, Halsall, et al., 2001; Denker et al., 2003b). In a similar study on SiGe islands, Usami et al. (2000a) found that while the first layer contains mainly dome-shaped islands, the second layer contains more pyramids.

The modulation of surface strain energy due to buried islands also leads to nearly periodic island arrangement for certain growth conditions, as has been observed experimentally with TEM and AFM by Teichert et al. (1996) in SiGe/Si superlattices. Increasing the number of periods, the island array becomes more periodic and the dispersion of the island sizes decreases, while the average size itself increases. This is shown in Fig. 15, with AFM images of the first and twentieth period in a $\mathrm{SiGe/Si}$ superlattice. Tersoff et al. (1996) gave a quite simple theoretical explanation of this effect, based on the assumption that the islands nucleate at the growing surface in local minima of the chemical potential for adatoms. These minima define a tessellation of the surface. The size of an island is proportional to the area of the corresponding Voronoi polygon, where the adatoms building up the island are collected. Neglecting the sur-

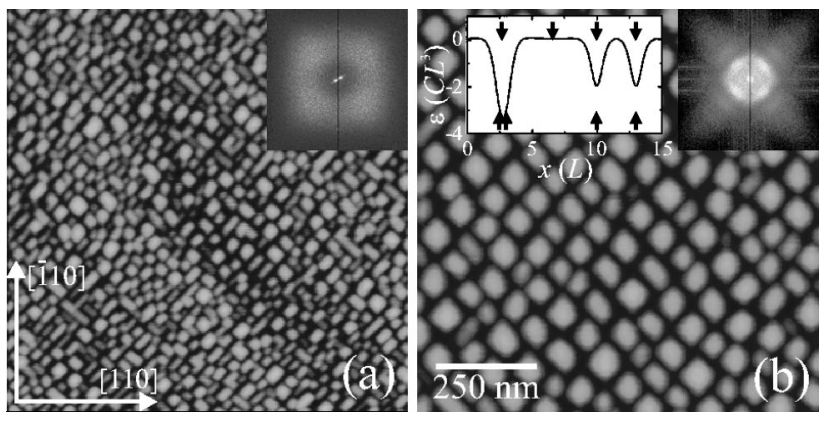

FIG. 15. AFM images of the surfaces of the $\mathrm{Si}_{0.25} \mathrm{Ge}_{0.75}$ layer in a $\operatorname{SiGe}(25 \AA) / \operatorname{Si}(100 \AA)$ superlattice and their power spectra (insets in upper right corners): (a) the first period (at the substrate); (b) the 20th period of the superlattice. From Teichert et al., 1996. The inset in the upper left corner of panel (b) shows the distribution of the surface stress that affects the chemical adatom potential. Buried islands give rise to deep stress minima above them. In addition, shallow minima appear between the deep minima, if the island distance is large enough. From Tersoff et al., 1996.

face curvature in Eq. (2), the profile of the chemical potential at the surface is entirely determined by the elastic energy density at the surface and consequently by the surface stress due to the buried islands. In the case of elastic isotropy, a local minimum of the chemical potential appears at the surface exactly above a buried island. Therefore the islands are arranged in vertical columns. The improvement of lateral periodicity can be qualitatively explained by the fact that an additional minimum of the chemical potential occurs between the islands if their distance is larger than the optimal one [inset in Fig. 15(b)]. Monte Carlo simulations were carried out for a one-dimensional model, indicating that during multilayer growth the island array indeed becomes more periodic and the dispersion of the island sizes decreases with an increasing number of island layers. The model has been extended to two dimensions and for islands of finite sizes by Liu et al. (1999) and Lam and Tan (2001). Similar results have been obtained by Zhang et al. (1999), who simulated island growth in a multilayer using a growth equation similar to Eq. (1), taking the surface stress due to buried islands into account. Daruka, Barabasi, et al. (1999) replaced the continuum approach for the calculation of surface stress by an atomistic simulation and obtained nearly the same results. Hence, for the purpose of nucleation simulation, the continuum model is sufficient.

Priester (2001) demonstrated that the potential minima for single adatoms calculated by Tersoff are actually too shallow to represent nucleation sites. However, these minima are efficient for organizing large adatom clusters, thus slightly enhancing the clustering probability in the positions of potential minima.

The degree of the vertical correlation of island positions can be quantified introducing the vertical pairing probability $P_{P}$ of islands (see Xie et al., 1995; Kienzle et al., 1999; Gonzalez et al., 2001). Growth simulations presented by Xie et al. (1995) showed that $P_{P}$ decreases with 


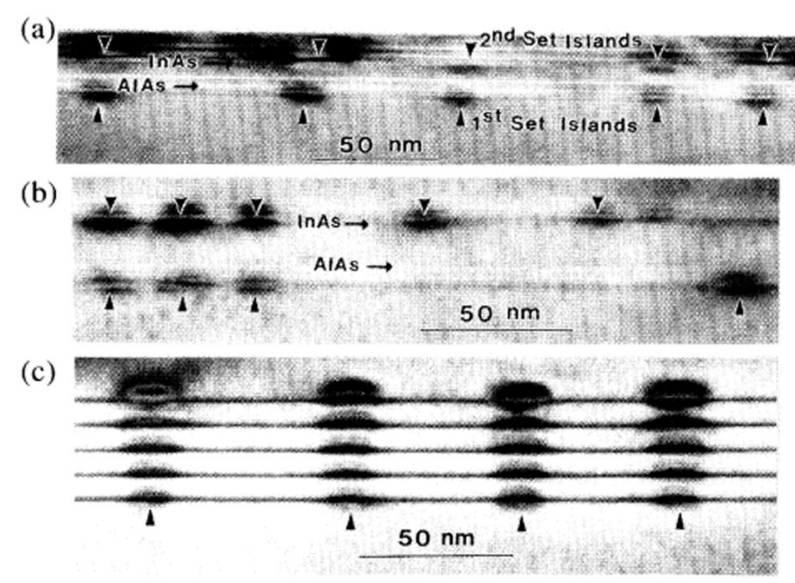

FIG. 16. InAs/GaAs island layers: (a) cross-section TEM images of InAs/GaAs double layers with spacer thickness of 46 ML's; (b) spacer thickness of 92 ML's; (c) perfectly correlated InAs/GaAs superlattice with 36-ML-thick GaAs spacers. From Xie et al., 1995.

increasing spacer thickness between the island layers (Fig. 16). For thin spacers, $P_{P}$ approaches unity; however, very thin spacers cause unfavorable island coarsening (Kienzle et al., 1999), and an optimum between island alignment and island size distribution has to be found. Meixner and Schöll (2003) determined $P_{P}$ using a kinetic Monte Carlo simulation of island nucleation, considering the influence of local surface stress on the hopping probability of adatoms. The results are plotted in Fig. 17. The pairing probability increases with the number of layers. Its dependence on layer thickness is more complicated. For thin spacers the probability is larger than 0.5 , indicating a vertical island correlation. For a certain thickness, a transition is observed from a correlated to an anticorrelated growth $\left(P_{P}<0.5\right)$ and finally the vertical correlation disappears for thick spacers $\left(P_{P}=0.5\right)$. Anticorrelation is also observed for coverages below $20 \%$. The pairing probability has been determined from grazing incidence $\mathrm{x}$-ray diffraction for $\mathrm{Ge} / \mathrm{Si}$ superlattices (Kegel et al., 1999b; Stangl, Roch, et al., 2000) as well as for InAs/GaAs multilayers (Gonzalez et al., 2001). Migliorato et al. (2001) used photolumines-

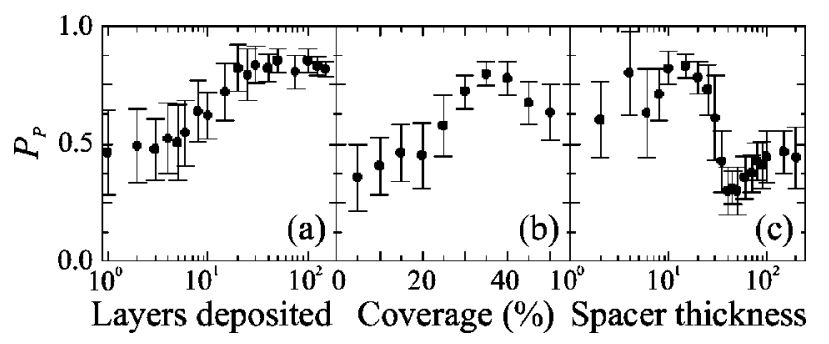

FIG. 17. Vertical pairing probability $P_{P}$ calculated by a kinetic Monte Carlo, method: (a) dependence on the number of deposited layers for constant spacer thickness of 15 ML's; (b) dependence on coverage for constant spacer thickness of $10 \mathrm{ML}$ 's; (c) dependence on spacer thickness for a coverage of 0.35 and 20 periods. From Meixner and Schöll, 2003.
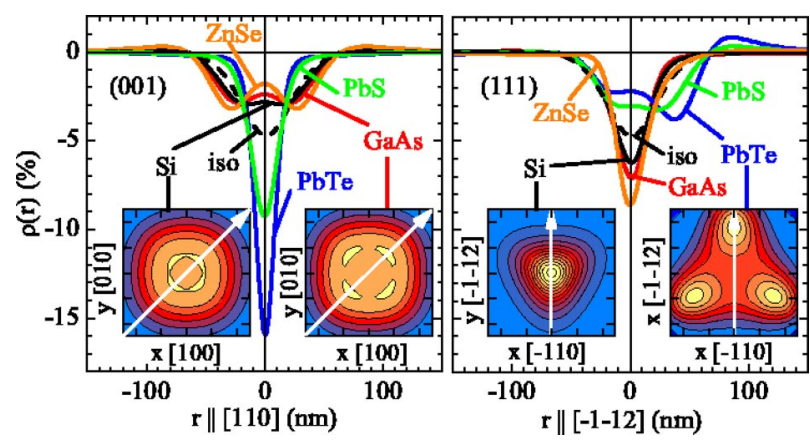

FIG. 18. (Color in online edition) Surface distribution of the chemical potential of an adatom calculated for various materials and surface orientations. The insets show the calculated 2D distributions, the main graphs depict the calculated distributions along the arrows in the insets. Only the relative change in the chemical potential with respect to the value for a homogeneously strained epitaxial layer is plotted. The light color in the insets corresponds to a minimum of the chemical potential.

cence to determine the vertical pairing of InAs islands embedded in GaAs.

In a series of papers (Yang, 2003; Yang and Tewary, 2003) another quantity has been used to describe the correlation of the island positions at different interfaces, namely, the elastic energy release rate. This quantity is defined as the change in the elastic energy of the whole system caused by a mass transport of a unit volume from the wetting layer to the volume of growing island. The maximum probability of the nucleation of an island corresponds to a local minimum of the elastic energy release rate Yang and Tewary (2003) used this approach to describe the vertical and lateral correlations of the positions of InAs islands.

\section{Oblique correlation}

A nonvertical alignment of the islands in a superlattice has been reported for the $(\mathrm{Cd}, \mathrm{Zn})$ Se system by Strassburg et al. (1998), and Krestnikov et al. (1999) as well as in PbSe/PbEuTe superlattices (Springholz, Holý, et al., 1998, 2002; Springholz, Pinczolits, et al., 2000a, 2000b, 2001; Springholz, Stangl, et al., 2000). This arrangement can be explained by the elastic anisotropy of the host lattice surrounding the buried islands. Due to this anisotropy, the chemical potential of a migrating adatom at the surface can exhibit several local minima, where the probability of island nucleation is enhanced.

Holý, Springholz, et al. (1999) systematically investigated the oblique alignment of islands for various material systems. An island buried below the surface is a stress source affecting the elastic energy density at the surface. Depending on the elastic anisotropy of the host lattice and on the orientation of the surface, different patterns of the energy density distribution can be obtained. The positions of the energy density minima at the surface are determined mainly by the elastically soft directions. Figure 18 shows the distribution of the elastic energy density at the surface calculated for various materials and two surface orientations. For instance, at a 


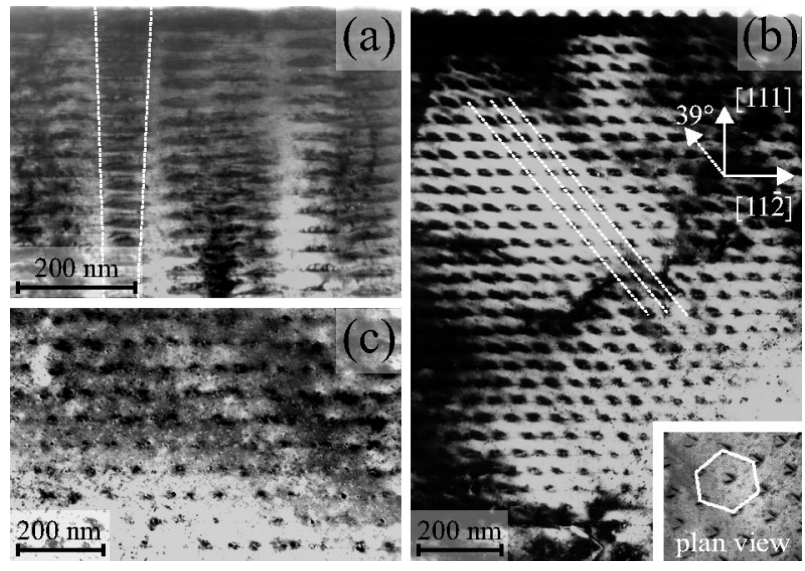

FIG. 19. Cross-sectional TEM images of $\mathrm{PbSe} / \mathrm{PbEuTe}$ superlattices with different thicknesses of the PbEuTe spacers: (a) $37 \mathrm{~nm}$; (b) $45 \mathrm{~nm}$; (c) $60 \mathrm{~nm}$. The inset in (b) shows the hexagonal in-plane arrangement of islands. From Springholz, Pinczolit, et al., 2000a.

(001) surface of a zinc-blende-type lattice, one central minimum or four minima of the chemical potential can be found, depending on the degree of elastic anisotropy. The resulting island arrangement depends also on the mean island size. If the distance between the minima is larger than the island size and the minima are sufficiently deep, an island buried below the surface induces the nucleation of four islands at the surface. In a periodic superlattice, this situation leads to a centered tetragonal lattice of islands, which corresponds to the type of correlation reported for CdSe island superlattices by Krestnikov et al. (1999). Such a vertical anticorrelation was also predicted for 2D islands by Shchukin et al. (1995, 1998) and Shchukin and Bimberg (1999).

If the distance between minima is smaller than the mean island size or if the minima are not sufficiently deep, the migrating adatoms do not "feel" the separation between these minima, but only one minimum instead, which results in a vertical correlation of the island positions. In this case, the lateral island correlation within the same interface is much weaker and is caused rather by maxima of the chemical potential in elastically hard directions. This is the case for a $\mathrm{SiGe} / \mathrm{Si}$ superlattice on $\mathrm{Si}(001)$, or InAs in $\mathrm{GaAs}(001)$, since the elastic anisotropy of these materials is relatively small (see Fig. 16).

Another interesting case is the (111) surface of a rocksalt-type lattice. Here, three local energy minima can be observed at the surface, which may give rise to a trigonal island stacking. This was observed for PbSe islands in $\mathrm{PbSe} / \mathrm{PbEuTe}$ superlattices, with a rather large elastic anisotropy of the host lattice and three wellpronounced minima. Figure 19 presents cross-sectional TEM images of three $\mathrm{PbSe} / \mathrm{PbEuTe}$ superlattices with various $\mathrm{PbEuTe}$ spacer thicknesses. For thin spacers (a), the distance between the three minima is smaller than the average island size, which results in vertical island stacking. For intermediate spacer thicknesses (b), the minima are well separated and a trigonal island stacking
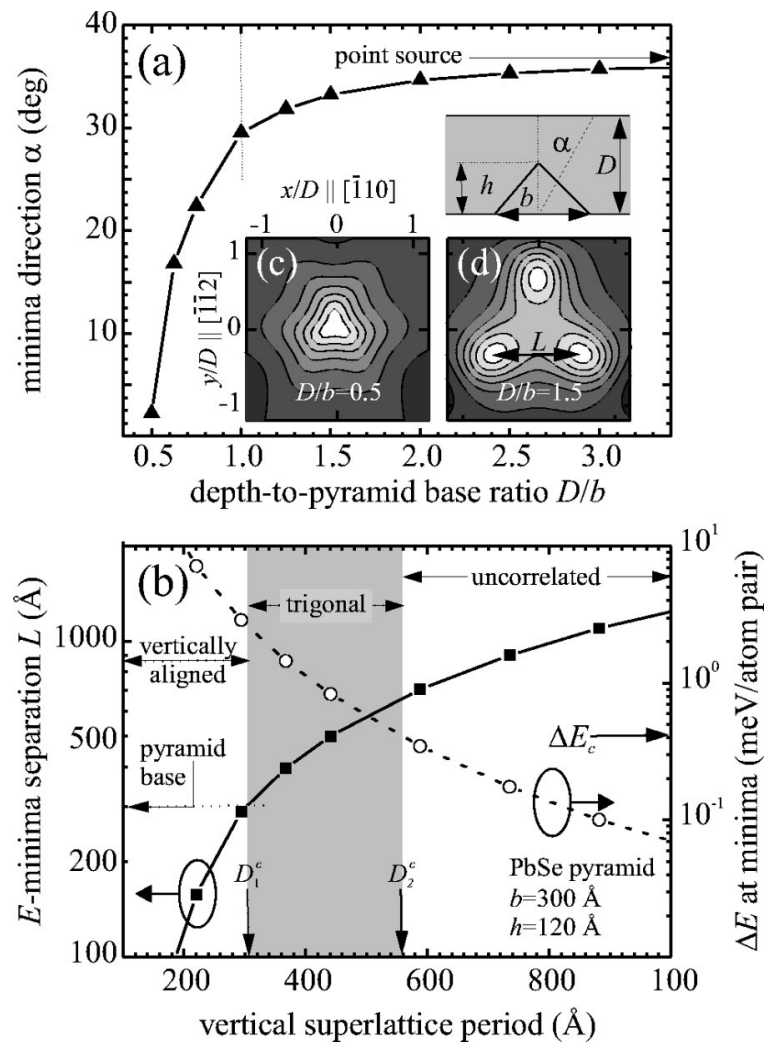

FIG. 20. Dependence of the stacking type on the PbSe island size (a) and on PbEuTe spacer layer thickness (b). The insets (c), (d) show the energy distribution on the surface for two different spacer thicknesses. From Springholz, Pinczolits, et al., 2000a.

appears. If the spacers are too thick (c), the distance between the minima is large but the minima are too shallow to affect island nucleation. In this case the island positions are completely uncorrelated. The various ordering types are plotted as a function of spacer thickness and lateral island size in Fig. 20. In the trigonal-stacking regime, i.e., for spacer thicknesses between 40 and $60 \mathrm{~nm}$, the island distances at a given interface scale linearly with the spacer thickness. Therefore it is possible to create artificial PbSe island "crystals" and tune their lattice parameter by varying the spacer thickness.

Under suitable conditions, the elastic anisotropy of the host material can cause an oblique arrangement of the dots even in systems where the dots are ordered mainly vertically. This was demonstrated by Heidemeyer et al. (2003) for a bilayer of InGaAs quantum dots grown on a prepatterned $\mathrm{GaAs}(001)$ surface. The distribution of the surface elastic energy caused by a buried InGaAs dot exhibits a deep central minimum and, in addition, four much shallower side minima (saddle points) in the $\langle 110\rangle$ directions. These minima occur due to the elastic anisotropy of the GaAs spacer layer. If this layer is too thin, the side minima play no role and the dots are perfectly vertically aligned due to the main minima. For thicker GaAs spacers, however, the side minima give rise to the nucleation of additional islands (satellite islands) obliquely correlated to the island underneath. 
If an alloy material is used as a spacer between the island layers, alloy decomposition due to the strain originating from a buried island may lead to an oblique replication of the island positions that cannot be explained by elastic anisotropy (Priester and Grenet, 2001). Growth kinetics can also lead to deviations from vertical correlation for materials with elastic properties that would predict vertical pairing. In TEM images, Sutter $e t$ al. (2001) observed a transition from vertical to oblique correlation in a $\mathrm{Ge} / \mathrm{Si}$ superlattice with $\mathrm{Ge}$ domes formed by 8.5-ML Ge and 40-nm-thick Si spacers, grown at $700^{\circ} \mathrm{C}$. This transition is due to an interplay of surface stress relaxation and surface curvature, influenced by the dome shape.

Oblique replication is also observed in $\mathrm{SiGe} / \mathrm{Si}$ multilayers exhibiting step bunching (Phang et al., 1994; Holý et al., 1998a, 1998b; Brunner et al., 2000). During growth, the strain field due to a step bunch in a buried strained layer influences the formation of step bunches in subsequent strained layers. As the strain fields associated with step bunches are anisotropic, the resulting correlation direction usually deviates from the growth direction.

\section{Self-organization process on prepatterned substrates}

Although the lateral self-ordering of islands can yield short-range ordered patterns with astonishing regularity, it does not result in true long-range ordering. Consequently, island nucleation on lithographically prepatterned substrates has been studied intensively. On prepatterned surfaces, similarly to nonpatterned substrates, the nucleation of the islands is affected by the surface curvature and the surface stress. If no mismatch stress is present, islands nucleate preferentially in places with negative curvature, i.e., in trenches or pits. If the local lateral lattice parameter at the apexes of the surface corrugation is closer to the intrinsic lattice parameter of the capping layer, for instance due to a buried stressor layer, the islands prefer to nucleate there (Lee et al., 2000), provided the adatom diffusion length is at least comparable to the trench or pit distance.

Various approaches have been followed so far:

(i) Ge island deposition on mesas grown into prepatterned $\mathrm{SiO}_{2}$ windows on $\mathrm{Si}$ has been used by Kamins and Williams (1997) and Jin et al. (2000). By properly adjusting the window size, a controlled arrangement of Ge islands can be realized (see, for instance, Kim et al., 1998; Vescan and Stoica, 2002). This local epitaxy works particularly well for gas-source molecular-beam epitaxy (MBE) or chemical vapor deposition.

(ii) Shallow etching of trenches in $\mathrm{Si}$ and subsequent overgrowth with a $\mathrm{Si} / \mathrm{SiGe}$ multilayer, followed by Ge nucleation at the strain-modulated surface, results in one-dimensional arrays of ordered islands on an essentially flat surface (Schmidt, JinPhillip, et al., 2000).

(iii) STM-induced pits were used by Yang et al. (2003)

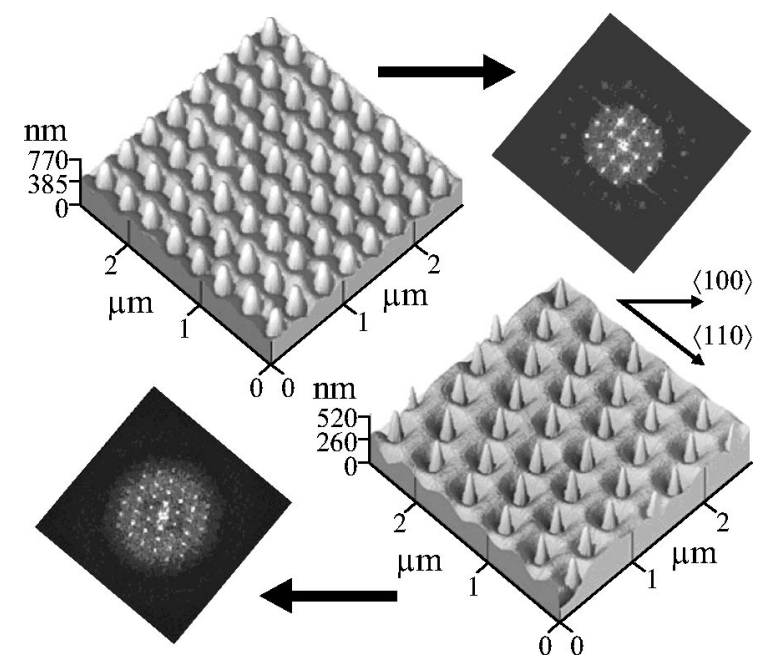

FIG. 21. AFM images of the surface of a Ge layer grown on lithographically prepatterned $\mathrm{Si}(001)$ substrates. In the sample shown at top left, the islands are arranged into a regular array along two orthogonal $<110\rangle$ directions. In the sample shown at bottom right, the unit vectors of the $2 \mathrm{D}$ array of pits are oriented along $<100>$ and $<110>$ directions, leading to a $45^{\circ}$ island alignment. Reprinted with permission from Zhong and Bauer, 2004, (C) 2004 American Institute of Physics.

to align InAs islands on GaAs/InAs heterostructures grown by MBE.

(iv) Direct growth of Ge in 2D periodically etched pits (Zhong et al., 2003) results in long-range-ordered island structures, as shown in Fig. 21. Nucleation occurs at the intersection of the side facets within the pits. This technique allows for a precise placement of individual islands. Similarly, InAs islands were grown on square-patterned GaAs substrates. An InGaAs layer buried by a GaAs/AlGaAs/GaAs spacer layer acts as a stressor for subsequent InAs island nucleation (Nötzel, 1996; Konkar et al., 1998; Lee et al., 2000, 2001). Recently, Heidemeyer et al. (2003) and Schmidt, Kiravittaya, et al. (2002) succeeded in depositing InAs islands in two-dimensionally arranged holes with a separation of $200 \mathrm{~nm}$ on a GaAs(001) surface.

(v) Laterally ordered $\mathrm{Ge}$ islands have also been grown on graded $\mathrm{SiGe}$ buffer layers on $\mathrm{Si}$ substrates. A regular array of buried misfit dislocations produces a regular strain pattern on the buffer surface which controls the nucleation of Ge islands (Shiryaev et al., 1997; Teichert et al., 2000). A drawback is the high density of threading dislocations (above $10^{6} \mathrm{~cm}^{-2}$ ) due to the graded buffer.

(vi) Combining the techniques of molecular-beam epitaxy growth on GaAs and in situ etching with $\mathrm{AsBr}_{3}$, Songmuang et al. (2003) fabricated closely spaced self-assembled InAs quantum dots, which they named "quantum-dot molecules." The num- 
ber of dots per nanohole can be varied between 2 and 6 . These pits are formed above capped InAs islands through strain-enhanced etching.

For Both Ge islands on $\mathrm{Si}$ and InAs islands on GaAs, growth on prepatterned substrates allows for the fabrication of both laterally and (in island multilayers) vertically ordered islands, i.e., regularly spaced columns of self-assembled islands. These represent artificial 3D island "crystals" embedded in a matrix material, in which the lateral distance is determined by the prepatterning and the vertical one by the spacer layer.

Self-organized islands buried below a planar 2D quantum well can also act as periodic stressors inducing periodic confinement of carriers in the quantum well. Here, confinement is achieved only via strain fields, without lateral material boundaries (see Lundstrom et al., 1999).

Using organometallic chemical vapor deposition (OMCVD) techniques for the growth of GaAs-based nanostructures in e-beam lithographically defined V-shaped grooves, Dwir et al. (2003) fabricated quantum wires and quantum dots with an excellent uniformity. These structures can serve as model systems for the study of the optical properties of low-dimensional systems. The mechanisms of self-ordering in nonplanar epitaxy of semiconductor nanostructures were studied theoretically by Biasiol et al. (2002). In this model the size-dependent lateral diffusion arises from the gradients in the chemical potential, and capillarity fluxes determine the different growth rates on the various facets that constitute the grooves of different orientations. These capillarity fluxes establish the self-limiting profiles of the nanostructures, which evolve either at the bottom of the grooves or on the tops of the mesas.

\section{EXPERIMENTAL METHODS}

In this section, we shall present an overview of techniques routinely applied for the characterization of semiconductor nanostructures. Each method has certain strengths and limitations, thus a combination of methods is usually required to obtain a rather complete structural characterization. Here, "complete" means that the shape, the distribution of chemical composition, and the strain fields within and around self-assembled nanostructures are determined in the analysis. Further, the mutual positions in an ensemble of such structures are of importance. Knowing all these, one can predict the electronic and optical properties, which are finally relevant in applications. Correlating these properties with certain growth conditions is the first step towards understanding growth and hence being able to engineer structures with desired properties. Because to our knowledge no extensive review on $\mathrm{x}$-ray scattering from semiconductor nanostructures exists, in this section we put the main emphasis on x-ray-based methods.

It might seem quite obvious which combination of methods is appropriate. The questions of how big islands are and what their shape is can be answered by AFM or
STM in the case of uncapped islands. Cross-sectional STM (XSTM) and TEM are powerful tools for the investigation of buried islands. In many cases, either method may be used and will yield similar results. X-ray diffraction (XRD) or photoluminescence may also be used to determine size and shape, but in general the resolution of AFM, STM, and TEM is higher. On the other hand, XRD and photoluminescence measure average properties of many islands. X-ray diffraction is very powerful for measuring strain fields, and from photoluminescence ground-state transition energies are obtained.

However, quantitatively determining the structural properties of a sample often turns out to be rather demanding with either method. An important example is the chemical composition. Using XRD or highresolution TEM with image analysis techniques, one can determine the distances between atoms or unit cells, but these depend not only on composition, but also on the strain state. Cross-sectional STM has the same limitation in evaluating the bulging of cleavage planes, as does photoluminescence, in which the energy levels in nanostructures depend on both composition and strain. A clear separation of these quantities requires model assumptions to interpret the results, and the calculation of strain fields from such models is a common task in the analysis of data obtained from very different methods. Strain simulations are therefore very important and are discussed separately in Sec. IV.

For the determination of statistically relevant properties, averaging over many individual nanostructures is necessary. Here, STM and TEM often cannot investigate large enough areas to yield quantitative data. With AFM, comparatively large areas may be scanned, but often increasing the scanning area means simultaneously decreasing spatial resolution. Photoluminescence and XRD investigate intrinsically large ensembles of islands, so that a good statistical average is obtained automatically, whereas the quantification of fluctuations is sometimes difficult.

In the investigation of correlations of the mutual positions of nanostructures, XRD is well suited to obtain quantitative results. AFM and TEM have also proven powerful for this purpose.

\section{A. Atomic force microscopy}

Atomic force microscope measures the force between the sample surface and a fine tip with a typical radius of less than $10 \mathrm{~nm}$. The force is measured either by the bending of a cantilever on which the tip is mounted (contact mode), or by measuring the change in resonance frequency due to the force (tapping mode). With a typical resolution of several nm laterally and several $\AA$ vertically, AFM is ideally suited to characterize the shape of self-assembled islands. For large scan sizes up to 100 $\times 100 \mu \mathrm{m}^{2}$, the lateral arrangement and correlation of island positions can also be obtained. With AFM any surface can be investigated; almost no sample preparation is required. Even material and strain sensitivity can 

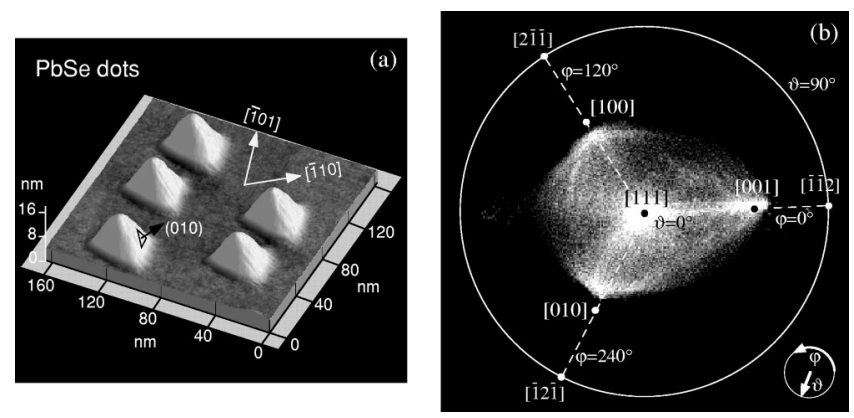

FIG. 22. PbSe islands with $\{001\}$ facets: (a) AFM image of the top surface of a $\mathrm{PbSe} / \mathrm{PbEuTe}$ island multilayer grown on $\mathrm{BaF}_{2}(111)$; (b) polar plot, showing the angular distribution of surface normals.

be achieved. A drawback of AFM is that only structures on a surface can be investigated. Furthermore, most semiconductor materials oxidize under ambient conditions, so that, strictly speaking, the AFM images usually show the surface of this oxide. When obtaining quantitative data such as island diameter and height, this has to be kept in mind, as well as the fact that the image is actually a convolution of the sample's surface morphology with the shape of the microscope tip.

Examples of the quantitative analysis of AFM images are shown in Figs. 22 and 23, showing the top surface of $\mathrm{PbSe} / \mathrm{PbEuTe}$ multilayers grown on $\mathrm{BaF}_{2}(111)$ substrates. PbSe forms triangular pyramids with $\{100\}$ side facets, as is shown in Fig. 22(a). In order to measure the sidewall angle, a polar plot of the orientation distribu-
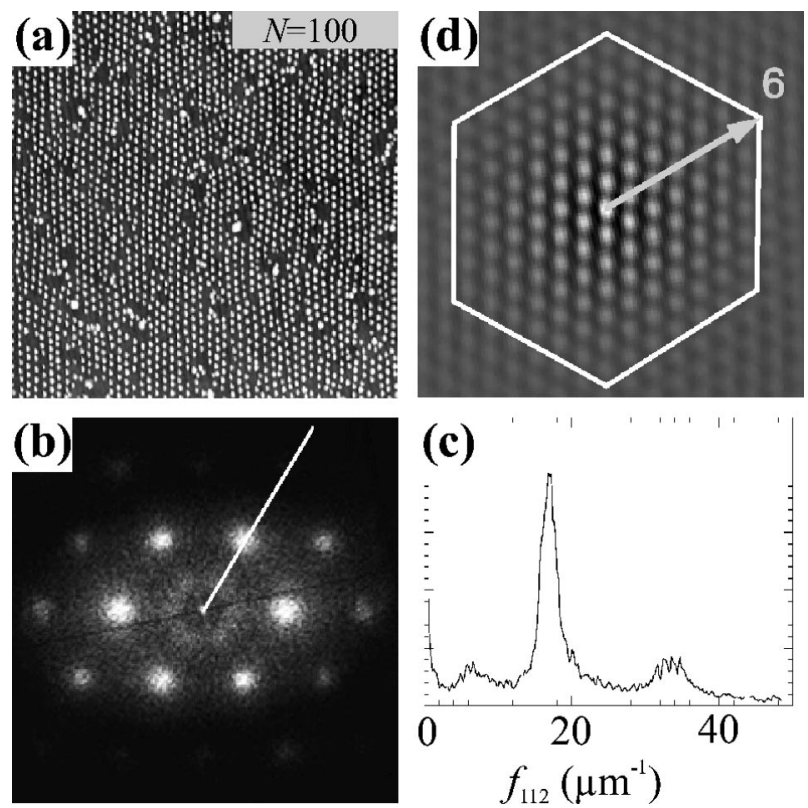

FIG. 23. Lateral island correlation: (a) AFM image (3 $\times 3 \mu \mathrm{m}^{2}$ ) of the top surface of a $\mathrm{PbSe} / \mathrm{PbEuTe}$ island multilayer; (b) Fourier transform of the image; the white line indicates the [112] direction; (c) the corresponding line profile with maxima due to island position correlation; (d) the autocorrelation function. Islands are arranged in a regular array up to the sixth-nearest neighbor.

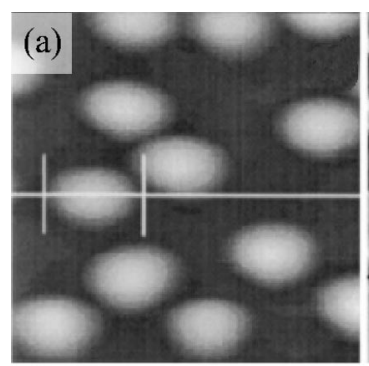

(b)

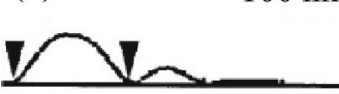

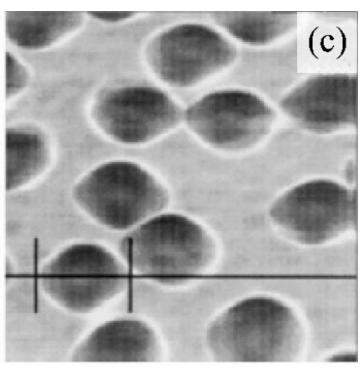

(d)
FIG. 24. Conventional topography (a) and ultrasonic force microscopy (c) images of SiGe islands on $\mathrm{Si}(001)$. (b) and (d) are the respective line profiles. The island height in (a), and (c) is about $15 \mathrm{~nm}$. From Kolosov et al., 1998.

tion of normal vectors to surface elements has been calculated (b). The lateral ordering can also be analyzed by AFM. In Fig. 23(a), a pronounced hexagonal in-plane arrangement of PbSe pyramids is evident. The Fourier transform of the image is shown in Fig. 23(b); panel (c) displays a line scan through (b) along the [112]] direction. Well-resolved satellite peaks illustrate island ordering. Fourier-transforming this image once more leads to the autocorrelation function shown in panel (d). Here shortrange island ordering is obvious, with a domain size including islands up to six times the next-nearest-neighbor distance (for a certain cutoff in autocorrelation contrast). Evaluating AFM images in such a way, a quantitative comparison of samples becomes possible.

A method different from standard AFM has been presented by Kolosov et al. (1998). Ultrasonic force microscopy uses a high frequency vibration of the sample, which imposes an additional force on the AFM tip. Hence in the associated image, besides the topographical information, the elastic response of the sample is also measured, which yields material sensitivity. Therefore ultrasonic force microscopy can distinguish between $\mathrm{Si}$ and Ge and is also sensitive to the strain, as strain influences stiffness. For islands formed by the deposition of $11 \mathrm{ML}$ 's of $\mathrm{Ge}$ on $\mathrm{Si}(001)$, ultrasonic force microscopy has detected the elastic relaxation of the island center, whereas the island circumference consists of strained Ge (see Fig. 24).

\section{B. Scanning tunneling microscopy and cross-sectional STM}

Scanning tunneling microscopy is based on controlling the tunnel current between a small tip and the sample surface. It is more restricted in terms of sample environment than AFM: only conductive samples can be investigated, and measurements usually have to be performed in ultrahigh vacuum. On the other hand, the tunnel current is sensitive to material composition and strain. Atomic resolution can be achieved laterally and vertically, making STM an ideal tool for the investigation of 
growing surfaces and facets at this scale, which can give insight into growth mechanisms. STM systems attached to a growth chamber allow for measurements without breaking the vacuum after growth. In some cases, STM is even applied in situ during growth and has been employed for the study of the onset of island formation. It is also used to monitor the transition between different island shapes. In situ studies are usually conducted at very small growth rates, and hence the results are not representative of growth at higher rates. However, growth at high rates is often studied at high temprature as well, which partly compensate each other. For postgrowth STM, the importance of annealing during cooling of the samples from the growth temperature has to be considered (the same is, of course, true for all other ex situ analysis techniques).

Qin et al. (2000b) presented STM images sensitive to the distribution of $\mathrm{Ge}$ on a $\mathrm{Si}(001)$ surface. While most STM images are obtained in the filled-state mode, they used empty-state imaging at low biases to detect intermixing sites for Ge at the $\mathrm{Si}$ surface. In the in situ experiment at rather low growth temperatures of $330{ }^{\circ} \mathrm{C}$, intermixing at very low Ge coverages between 0.02 and 0.2 ML's i.e., in the very first stages of Ge deposition and before islands form, was found to occur randomly across the surface, without a preference of steps or point defects as intermixing sites. So-called buckled dimers are the fingerprint of Ge incorporation sites (see also Fig. 2 in Sec. II.A).

The same authors applied a technique called atomtracking STM to investigate the diffusion of adsorbed Si-Ge mixed dimers on $\mathrm{Si}(001)$ (Qin et al., 2000a). At temperatures below $100^{\circ} \mathrm{C}$, the kinetics become slow enough for the diffusion of a mixed Si-Ge dimer to be traced with STM. Using a feedback loop over the $x$ and $y$ position, Qin et al. kept the microscope tip at the locally highest point of the surface. In this way, its position was monitored with time, recording the track of the dimer with a resolution of about $5 \mathrm{~ms}$. Si-Si, Si-Ge, and Ge-Ge dimers exhibited quite different kinetic behavior. While Si-Si dimers rotated between two orientations differing by $90^{\circ}$ from each other, Si-Ge mixed dimers were buckled, with one atom higher above the surface than the other, and they rotated between two states differing by $180^{\circ}$. Ge-Ge dimers were seen to quickly break up into one $\mathrm{Si}-\mathrm{Ge}$ dimer bound in the surface and one adsorbed to the surface. The experiment showed the rotation of the mixed dimers and the diffusion from one lattice site to another at different time scales, corresponding to activation barriers of 0.82 and $1.01 \mathrm{eV}$, respectively. It could also be clearly seen when the $\mathrm{Ge}$ atom in the mixed dimer exchanged with a $\mathrm{Si}$ atom of the surface, resulting in a bound mixed dimer and a $\mathrm{Si}-\mathrm{Si}$ adsorbed dimer with different rotation kinetics. Such an exchange was observed only in conjunction with hopping of the dimer to a different site.

Apart from structural information, low-temperature scanning tunneling spectroscopy has been used for a wave-function mapping of single electron states in InAs dots (Maltezopoulos et al., 2003). The $\mathrm{d} I / \mathrm{d} V$ curves reveal $s^{-}, p-, d$-, and even $f$-type states, and an asymmetry of the electronic structure, attributed to a shape asymmetry of the islands, along the two perpendicular $\langle 110\rangle$ directions.

The tunnel current in STM is sensitive only to a thin layer at a sample surface, and it therefore might seem that buried structures are beyond the scope of STM studies. Although the former is certainly true, the latter is not: cleaving samples and performing STM at the cleavage edge through the nanostructures (crosssectional STM) can reveal details on the inner structure of buried islands. Three effects have mainly been exploited in cross-sectional STM: first, using the element sensitivity of the tunnel current to determine the elemental distribution within the cleavage edge, one can measure the shape and composition of buried islands. Second, measuring atom spacings yields information on strain and composition. Third, due to elastic strain relaxation the cleavage face bulges, and the composition/ strain distribution in buried nanostructures is transformed into a height profile accessible to STM. As the position of an island relative to the cleavage plane influences the result, the measurement of many cleaved islands is required, together with model calculations. Furthermore, atomically smooth cleavage planes are required for this technique, which so far have been achieved only for III-V semiconductors.

The first applications of this method were by Lita et al. (1999), who found InAs segregation in island multilayers in GaAs(001), and by Eisele et al. (1999), who observed that islands with trapezoidal cross sections are larger in subsequent layers than in the first layer. Liu, Tersoff, et al. (2000) determined the InAs composition profile in similar samples and found an inverted-cone distribution of InAs, deduced from atom spacings. The bulging of the cleavage face [Fig. 25(a)] was measured to determine the In distribution and the shape of InAs islands in GaAs by Bruls et al. (2003). The In content increased towards the island apex, but In segregation was less pronounced above the islands than above the wetting layers. The dots in the second and subsequent layers formed at $z$ positions slightly shifted to larger $z$ values, causing a deformation which became more pronounced the higher the island was in the stack. While the InAs growth rate in the islands seemed to remain constant laterally, the deformations were enhanced by an inhomogeneous GaAs growth rate, lower above the island center than in the outer regions, resulting in a wingshaped island cross section in the uppermost layers. Moreover the GaAs spacer thickness was not uniform, but decreased directly above the dot (less pronounced for the first two islands in the stack). Bruls et al. (2003) propose that both the local strain and thermodynamics play a role.

Figure 25(b) shows a comparison of the measured and simulated cross-sectional STM profile for an InAs island in GaAs (Grandidier et al., 2000). Recording topographic images simultaneously with current-voltage 

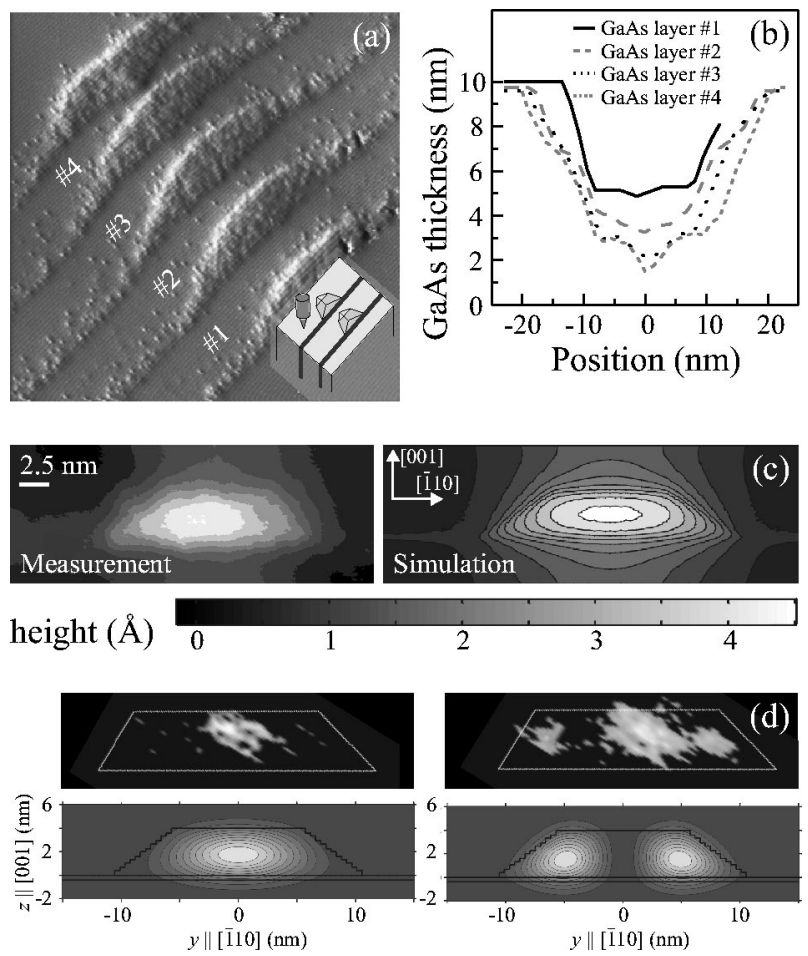

FIG. 25. Cross-sectional scanning tunneling microscopy: (a) XSTM image of a stack of InAs islands in GaAs; (b) the thickness of the GaAs spacers as a function of lateral position; (c) comparison between a measured and simulated height profile for a similar sample; (d) the electronic wave function measured at two different tip biases, compared to simulations for the ground state and the first exited state. Panels (a) and (b) from Bruls et al., 2003. Panels (c) and (d) from Grandidier et al., 2000.

characteristics, it is possible to measure the carrier distribution within an InAs island with STM. Simulations of the conduction-band ground state and first excited state of a pure InAs island correspond well with images obtained at tip biases of $+0.69 \mathrm{~V}$ and $+0.82 \mathrm{~V}$ [Fig. 25(d)], showing that the wave functions in such islands are indeed atomlike. Only electrons between the Fermi energies of the sample and the tip contribute to the image. In the left panel, these are electrons in the ground state; in the right panel, electrons from both the ground state and the first excited state contribute to the measured distribution. At $+0.69 \mathrm{~V}$ only $s$ electrons contribute, for $+0.82 \mathrm{~V} s$ and $p$ electrons. From a careful comparison of the measured height profile of cleavage face bulging with finite-element simulations of the elastic relaxation, Bruls, Vugs, et al. (2002) determined the strain and composition distribution of InAs islands in GaAs (Bruls, Koenraad, et al., 2002). The buried InAs islands have the shape of truncated pyramids. Assuming different InAs profiles in the finite-element simulations, island intermixing was found with a GaAs composition decreasing linearly from 0.4 at the base to 0 at the top of the islands. The corresponding lattice parameter distribution in growth direction is shown in Fig. 26. It shows directly the increase of compressive strain in the GaAs matrix above and below the islands.

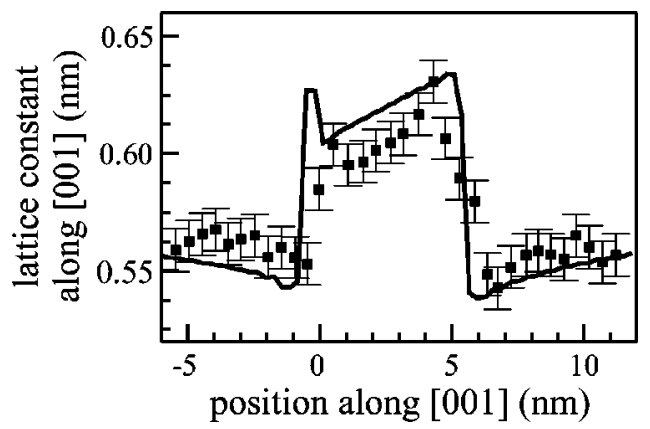

FIG. 26. Lattice parameter in growth direction in an InAs island: $\mathbf{\square}$, obtained from cross-sectional STM; solid line, obtained from a finite-element simulation assuming an In content increasing from island base to island apex. From Bruls, Vugs, et al., 2002.

An interesting combination of STM and electroluminescence was used by Jacobs et al. (2003), who injected carriers via the microscope tip locally into the surface of a GaAs $p-i-n$ sample with InGaAs quantum dots and were thus able to measure the electroluminescence of individual islands with high spatial resolution.

\section{Transmission electron microscopy and scanning electron microscopy}

Although the preparation of thin specimens is more elaborate than for other techniques, which makes TEM essentially an ex situ characterization technique, it is widely used due to its very high spatial resolution and sensitivity composition. Transmission electron microscopy can be performed either on thin slices parallel to the sample surface (plan-view TEM) or on crosssectional slices. Hence buried islands can be well examined by TEM, with some restrictions due to specimen preparation: in many cases, the lateral island diameter is comparable to the slice thickness. Then the observed properties, which are averages over the slice thickness, depend on the location of an island or the part of an island within the slice. The image contrast depends on different quantities, material (composition) but also strain, as TEM images are obtained from diffraction patterns of high-energy electrons. Therefore image analysis is often not straightforward but requires elaborate image analysis techniques and/or model calculations. Compared to other techniques, usually very small areas are investigated, so that no statistically averaged values can be obtained.

In the following, two kinds of TEM investigations are discussed. First we consider the use of conventional TEM to obtain, morphological information such as island shape and correlation of island positions, as well as some compositional information. Then atomically resolved images are analyzed, and from the position of unit cells strain and composition information is derived. 


\section{Morphology}

From TEM and AFM images of SiGe islands on $\mathrm{Si}$ deposited with different $\mathrm{Ge}$ coverages from 3.5 to $14 \mathrm{ML}$ 's, and at growth temperatures $T_{\mathrm{g}}$ from 450 to $600{ }^{\circ} \mathrm{C}$, Chaparro et al. (1999) found an increase of average island size with growth temperature. For low $T_{\mathrm{g}}$, larger islands were domes, whereas for high $T_{\mathrm{g}}$ they were pyramids, due to enhanced interdiffusion. Energydispersive x-ray analysis showed $\mathrm{Si}$ alloying into islands. A similar result was obtained in a plan-view TEM study yielding the shape and strain of annealed samples by Henstrom et al. (2000).

Kienzle et al. (1999) investigated the correlation of island positions in multilayers using cross-sectional TEM. Bright-field images exhibited material contrast, while dark-field images were sensitive to the strain fields. For Si spacers less than $40 \mathrm{~nm}$ thick, very good vertical alignment of island positions was observed, with an increase of island sizes in subsequent layers. Thick spacers resulted in poor or no alignment, but no coarsening. Both effects were mediated by strain fields from islands in previous layers.

While island alloying during deposition has often been reported, the questions of how exactly this alloying takes place and how material transport occurs have seldom been addressed. Liao et al. (1999) investigated the MBE growth of $\mathrm{Ge}$ on $\mathrm{Si}(001)$ at $700{ }^{\circ} \mathrm{C}$. They observed that trenches were formed around the islands. The Si removed from the perimeter diffused into the growing islands. This alloying reduced the overall strain energy, which is believed to be the driving force for alloying. As $\mathrm{Si}$ interdiffusion is mainly from below the growing island, whereas the island apex can relax elastically, the Ge composition in an island is not uniform, but increases from bottom to top, as was found by an energydispersive $\mathrm{x}$-ray analysis (Liao et al., 2000). Trench formation seems to occur at higher substrate temperatures of around $700{ }^{\circ} \mathrm{C}$, whereas at a growth temperature of below $600^{\circ} \mathrm{C}$ interdiffusion leads to an extension of the $\mathrm{SiGe}$ island below the original $\mathrm{Si} / \mathrm{Ge}$ interface, but without trenches being formed (Liao et al., 2002).

\section{Digital analysis of lattice images}

In order to determine the strain and composition distribution, one can measure the unit-cell spacing and its local variations: the lattice parameter changes with composition and is, of course, also sensitive to strain. Atomically resolved images of nanostructures are filtered to enhance features with lattice periodicity and to suppress slow intensity variations (e.g., due to variations of slice thickness) and noise. In the digital analysis of lattice images, or DALI method, the unit cell positions are determined from these filtered images. Then the shifts of the unit cells with respect to a reference lattice are obtained, determined from the undisturbed lattice, in many cases in the substrate. As these shifts are cumulative and in most cases exceed the lattice parameter, it is necessary to shift the reference lattice from unit cell to unit cell

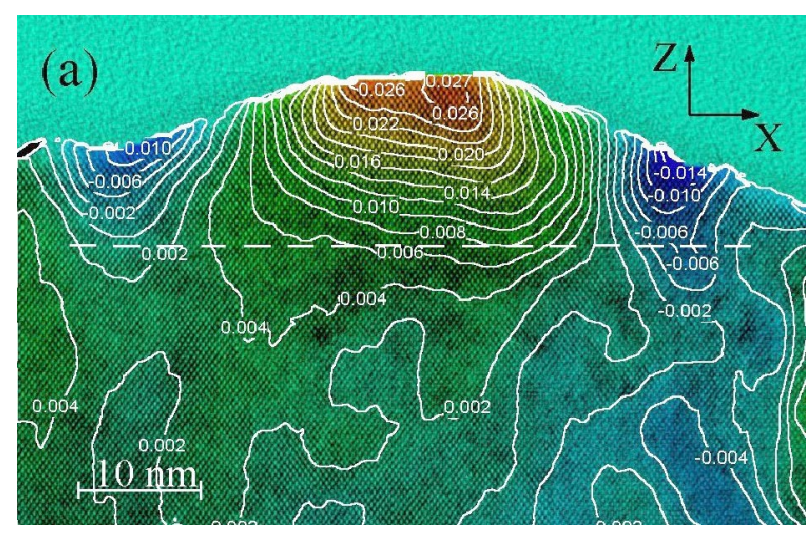

FIG. 27. (Color in online edition) Strain distributions $\varepsilon_{x x}$ obtained from TEM images of InGaAs islands in GaAs using the method of digital analysis of lattice images (DALI). From Kret et al., 1999.

and to determine the relative shift for each unit cell. Adding the relative shifts gives the total displacement field $\mathbf{u}$, from which the strain with respect to the reference lattice is also calculated.

As the lattice spacing is a function of both composition and strain, the analysis requires model calculations. Mostly, finite-element method calculations are used with assumptions on composition gradients to calculate the displacement fields. These are then compared to the experimentally obtained $\mathbf{u}$; however, it has to be considered that TEM yields data averaged over the slice thickness, so that the finite-element results need to be averaged as well. As the slices are very thin, their elastic relaxation needs to be considered, which requires a precise determination of slice dimensions.

Rosenauer et al. (1997) used the DALI method for the determination of the In profile in InGaAs islands grown on GaAs by MBE. Interdiffusion between InAs and GaAs occurred at a growth temperature of $560^{\circ} \mathrm{C}$, leading to an In content increasing from island bottom to top. For a sample in which pure InAs had been deposited, the average InAs content was found to be as low as $48 \%$. In a study of capped islands, additional dilution of InAs was observed (Rosenauer et al., 2000, 2001). After capping, a thick wetting layer with In-rich inclusions was left rather than a wetting layer plus islands. Obviously, the layers grew faster between the islands than on top of them, because GaAs was incorporated mainly where no elastic lattice relaxation took place. Together with a pronounced InAs segregation, the islands were embedded into a dilute InGaAs wetting layer.

Very detailed composition maps of InAs islands grown with $\mathrm{MBE}$ on $\mathrm{GaAs}(001)$ at a substrate temperature of $510{ }^{\circ} \mathrm{C}$ have been obtained by Kret et al. (1999; see Fig. 27).

In contrast, Carlsson et al. (1998) found no change in shape during capping for InP islands on GaInP grown by organometallic vapor-phase epitaxy. The island shape was obtained from conventional TEM images, while the strain was deduced by image analysis as above. Careful comparison of TEM images obtained in two orthogonal 
$\langle 110\rangle$ sample azimuths with finite-element calculations showed that also for very small structures linear elasticity theory may be applied.

For SiGe, which crystallizes in the diamond lattice, a different method for analyzing strain and hence the Ge composition in TEM images has been presented by Miller et al. (1999). Ge islands grown on $\mathrm{Si}(001)$ by chemical vapor deposition produce a moiré-like pattern in plan-view TEM images due to the different lattice parameters in the islands and the Si substrate. Recording dark-field images at the two beam conditions for the (220) and (400) diffracted beams, Miller et al. observed intensity fringes where $\mathrm{SiGe}$ islands were located. The fringe patterns were simulated, assuming an abrupt change in the lattice parameter, with the Ge composition and hence the in-plane strain as free parameters. With this method it was possible to obtain the Ge composition with a resolution of about $10 \%$. For samples with $\mathrm{Ge}$ deposited at $600{ }^{\circ} \mathrm{C}$ from $\mathrm{GeH}_{4}$ with $\mathrm{H}_{2}$ as the carrier gas, the results are consistent with pure $\mathrm{Ge}$ in the islands, which differs from results obtained by other groups.

\section{Composition evaluation by lattice fringe analysis}

An alternative method of deriving the chemical composition profile is lattice fringe analysis, presented by Rosenauer et al. (1998) and Rosenauer and Gerthsen (1999), which they demonstrated for InGaAs islands in GaAs. It uses particular imaging conditions, in which the sample is tilted by a few degrees off a low-index crystallographic plane. In this way, the extinction depth for electrons is increased from about $10 \mathrm{~nm}$ to over $100 \mathrm{~nm}$, and hence thickness variations do not play an important role in image analysis. The image is recorded with the [002] direction in the optical axis. Lattice fringes in the image result from the interference of (000), (002), and (004) diffracted beams. For the analysis, the amplitudes and phases of these three beams need to be evaluated. It turns out that only the amplitude of the (002) beam varies significantly with In composition [(002) is a chemically sensitive reflection in zinc-blende structures], and hence the ratio of intensities $I_{002} / I_{004}$ is a measure of the In composition. In the evaluation, the unknown imaging parameters such as defocus and sample thickness can be evaluated from the fringe pattern in an area without In. Then, the fringe pattern in the areas with In is fitted (Fig. 28), and the In content derived. In the case of InGaAs islands formed by the deposition of $2 \mathrm{ML}$ InGaAs with nominally $60 \%$ of InAs at $500{ }^{\circ} \mathrm{C}$, and capped with $\mathrm{GaAs}$, an In-rich layer with a homogeneous thickness of 3.4 ML, but with a laterally inhomogeneous In profile is observed. In-rich regions with a maximum In content of about $54 \%$ correspond to StranskiKrastanow-grown islands before capping with GaAs. Between those regions, i.e., where only a wetting layer existed prior to capping, the In content is only about $40 \%$. Both values are lower than the In content of the deposited material. A comparison between this method and the DALI method performed on the same TEM

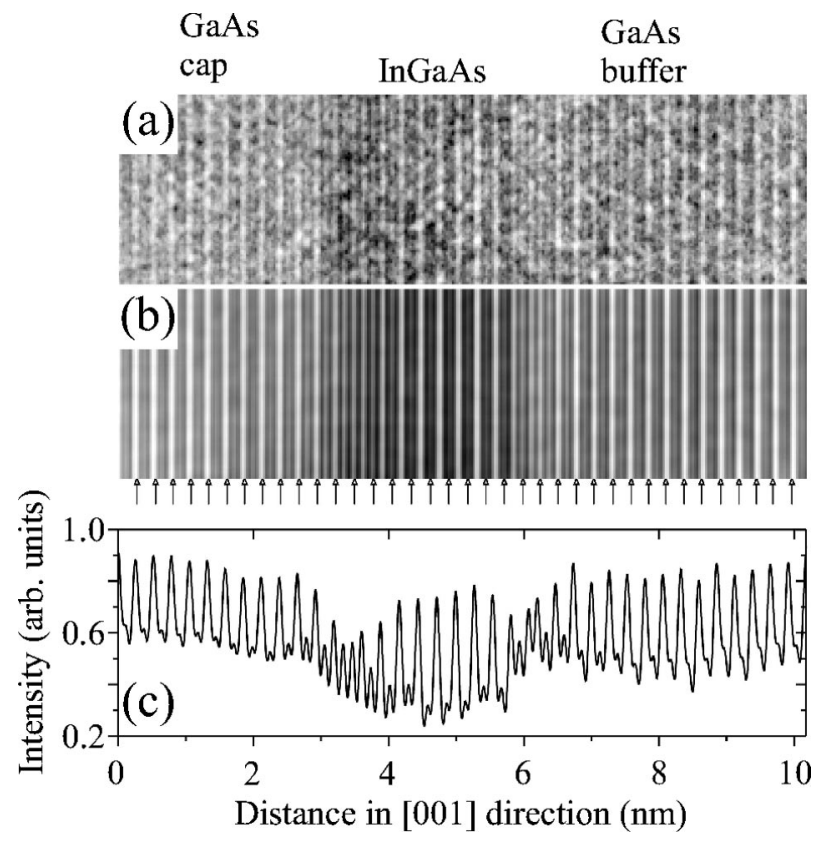

FIG. 28. Lattice fringe analysis of a high-resolution TEM image of an InGaAs layer embedded in GaAs. From Rosenauer et al., 1998, reprinted with permission of Elsevier. Copyright 1998.

images reveals a very good correspondence.

\section{Quantitative analysis of the information from transmission electron micrographs}

Under certain circumstances, a method sometimes known as QUANTITEM can be used for the analysis of chemical composition (Kisielowski et al., 1995). The particular advantage of this method is that the imaging conditions may be widely unknown, as the obtained intensity pattern is treated as an unknown function of the projected potential of the sample, and this function is determined during image analysis. Very simply, this quantitative analysis of TEM images determines the dependence of the measured intensity pattern on the projected potential. The latter is given mainly by the material-sensitive extinction length of electrons in the sample and by the sample thickness. The TEM image is split into unit cells, and each cell is digitized into $N=n$ $\times m$ points. The intensity of these points is considered a vector of length $N$. Three unit cells are selected as basis vectors, and all other unit-cell vectors are expressed as linear combinations of those vectors and can hence be written as three-dimensional vectors. This holds exactly for the two-wave case, i.e., for materials and imaging conditions in which only two Bloch waves are excited (having an intensity larger than 5\%), which is the case for many material systems in high-symmetry directions. From the three-dimensional representation of the unitcell vectors, which form a plane ellipse, the rate of change of projected potential with thickness can be deduced. This information can be exploited in various ways. For example, the local thickness of a sample with known composition can be extracted from TEM images. 

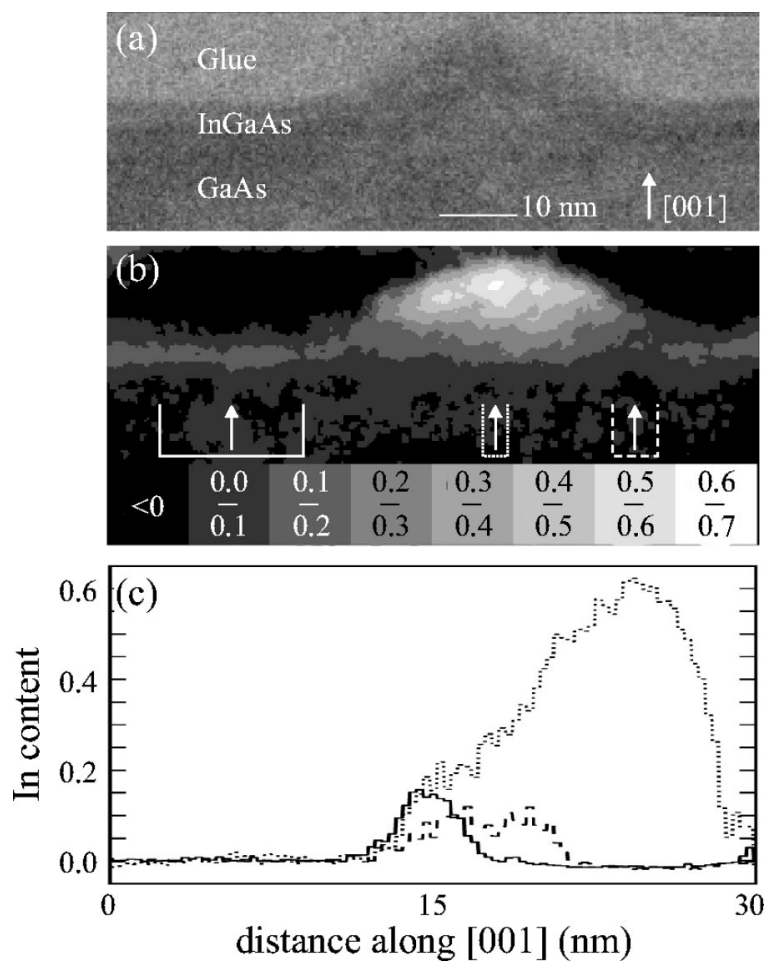

FIG. 29. Energy-selective imaging (ESI): (a) conventional TEM bright-field image of InAs islands in GaAs; (b) In concentration map obtained from ESI; (c) line profiles of In concentration along the growth direction averaged over the stripes indicated in (b). From Walther et al., 2001.

If the thickness is known, on the other hand, the chemical composition can be obtained. In the application to semiconductor islands, the specimen thickness is evaluated in regions outside the islands and interpolated to the islands themselves, and in this way the composition can be evaluated. A major limitation of this method is that the measured quantity is (as generally with TEM) the projected potential. If the actual potential varies along the electron-beam path, the relation between thickness, chemical composition, and projected potential cannot be obtained from the images directly.

\section{Energy-selective imaging}

In a study on the segregation of In during the deposition of $\operatorname{In}_{0.25} \mathrm{Ga}_{0.75} \mathrm{As}$ on GaAs, Walther et al. (2001) used energy-selective imaging, i.e., the comparison of TEM images taken at different electron energies below and above the In $M_{4,5}$ and Ga $L_{2,3}$ edges, to obtain the In and Ga distribution, as shown in Fig. 29. A series of samples was grown by $\mathrm{MBE}$ at $540{ }^{\circ} \mathrm{C}$. During growth, In segregation increased the surface In concentration, which triggered the formation of In-rich islands. The In content in the island apexes was found to be about $60 \%$, which is a lower limit for the actual content, as TEM always averages along the electron-beam direction. The authors estimate that after about $3 \mathrm{~nm}$ of InGaAs deposition, a stable surface forms with $85 \%$ InAs, which is required to initiate islanding. For In compositions of the deposited alloy below $25 \%$, no islanding has been observed.

\section{Scanning transmission electron microscopy}

In contrast to the conventional TEM technique, scanning transmission electron microscopy (STEM) uses a highly focused electron beam with a diameter of a few angstroms (James and Browning, 1998; Browning et al., 2001). Since this diameter is comparable with or smaller than the interatomic distance, the different atoms are irradiated incoherently so that the resulting image is not created by electron diffraction. Therefore the $Z$-specific information that can be obtained using the STEM method is not affected by the strain in the specimen.

The STEM method is frequently used for chemical analysis of cross sections of individual quantum dots. It has been applied to InAs/GaAs quantum dots (Murray et al., 1999; Roh et al., 2001; Yamaguchi et al., 2002), to $\mathrm{SiGe} / \mathrm{Si}$ dots (Dunbar, Bangert, et al., 2001), and to $\mathrm{CdSe} / \mathrm{ZnSe}$ systems (Kim et al., 2000). For the interpretation of the results, the location of an island relative to the slice is important. While the chemical composition profile along the center axis of the islands is rather insensitive to island shape [Zhi et al. (2001) reported an average In content between 63 and $71 \%$ for InAs islands in GaAs, depending on whether a pyramidal, hemispherical, or conical island shape was assumed in the data evaluation], in the outer regions of an island the assumed shape is of more importance. Here the quantitative analysis by TEM has very similar limitations and error bars to those of XRD (see below), which also often relies on model assumptions.

Usually, in TEM either plan-view or cross-section specimens are prepared. It is, however, also possible to prepare a very thin needlelike sample (see, for example, Ozasa et al., 2003) so that rotation of the specimen allows for a continuous change from planview to cross section TEM, and the properties obtained in either geometry can be compared for one specific island.

\section{Low-energy electron microscopy}

For the in situ analysis of self-assembled island formation during growth, low-energy electron microscopy (LEEM) has been employed (Bauer et al., 1991). This is a very surface-sensitive technique, as the low energy, in the range of 10-100 eV, of electrons backscattered from the surface, causes a penetration depth of only a few atomic layers. Images are formed using electron lenses from reflected and diffracted beams. The contrast in this technique depends on many factors, including surface material, electron energy, and local variations of the diffraction conditions, or surface morphology. The reflectivity is high for flat surfaces, and decreases with increasing density of monolayer steps (for steeper side faces). Hence flat terraces appear bright, while steep terraces appear dark.

Sutter and Lagally (2000) studied the formation of islands during deposition of $\mathrm{SiGe}$ with low Ge content to 


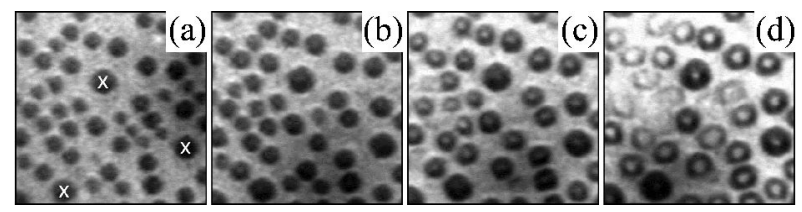

FIG. 30. Sequence of low-energy electron microscope (LEEM) images during capping of SiGe islands with Si: prior to Si deposition, and at 4.6-, 9.2-, and 13.8-ML Si cap. The image size is $1.5 \times 1.5 \mu \mathrm{m}^{2}$. From Sutter and Lagally 1998 .

tackle the question of whether islands form continuously [see also Tersoff et al. (2002)] or in a nucleation process. Very similar studies were performed by Tromp et al. (2000). For low Ge content (25\%), a roughening of the surface was observed, presumably due to the formation of a dense array of small 2D islands, followed by the formation of a small "cell" array at the surface, where the cell size varied inversely with the deposited alloy composition. The regions between the cells became bright, indicating a low step density, while the cells themselves gradually became darker, due to an increasing step density, corresponding to a mound or island slope becoming gradually steeper. This process continued until the angle reached that of $\{105\}$ facets. Beyond this point the surface evolved as $\{105\}$ faceted pyramids. Thus for low Ge content, the barrierless formation of islands could be confirmed using low-energy electron microscopy. The transition between Ge pyramids and domes through a series of states with an increasing number of highly inclined facets was investigated via this technique by Ross et al. (1999).

Using in situ low-energy electron microscopy, Sutter and Lagally (1998) also monitored the shape transition of self-assembled SiGe islands during capping with Si. The formation of a top (001) facet was observed, visible as bright spots in Fig. 30, as the island apex was dissolved by $\mathrm{Si}$ and the material transported to the side facets. Consequently, the island height was decreasing during overgrowth, while the base width was increasing. The $\{105\}$ facets were preserved at the sides. The shape change occurred only under Si flux and was not a ripening process, but the growth temperature had an influence on the material redistribution rate. The authors explained this shape evolution with a simple model including a dissolution rate of the topmost ML and an incorporation rate of $\mathrm{Si}$.

\section{E. X-ray scattering}

In x-ray scattering, intensity distributions in reciprocal space are recorded instead of real-space images, and in most cases the interpretation of experimental data requires model assumptions and fitting routines. As in electron microscopy, the incident beam is diffracted. Due to the lack of x-ray lenses, however, it is not possible to form an image out of the diffraction pattern; the pattern itself is recorded. As only intensities can be measured, the phase information on scattered $\mathrm{x}$ rays is lost, which is certainly a drawback of XRD. The measure-

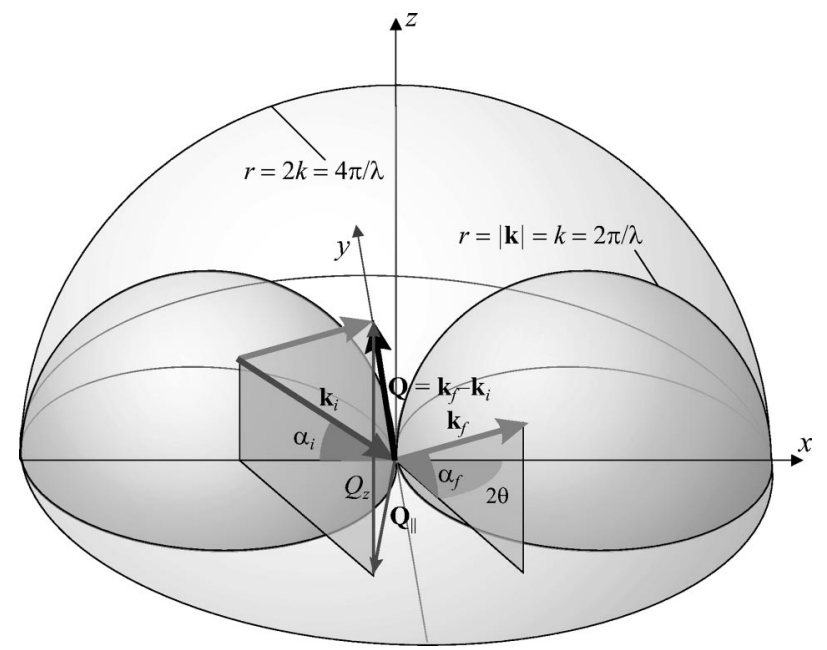

FIG. 31. Reciprocal-space sketch of a general scattering experiment. For a detailed description see the text.

ments usually require no special sample environment or preparation. The penetration depth of $\mathrm{x}$ rays between several $\mathrm{nm}$ and several hundred $\mathrm{nm}$ allows for the nondestructive investigation of both buried and surface structures. Choosing the proper scattering geometry, one can tune the penetration depth so that information is gained either about the surface or about the bulk. Different means of contrast can be used, e.g., differences in the refractive index are exploited in x-ray reflectivity and grazing-incidence small-angle $x$-ray scattering, while the strain fields are measured in coplanar x-ray diffraction and grazing-incidence diffraction. Differences in the atomic scattering factors and their dependence on energy can also be used in anomalous scattering experiments. The investigated sample areas are typically several tenths to several $\mathrm{mm}^{2}$, hence very good statistics on sample properties are reached. One particular strength of XRD is its very high strain sensitivity.

Commonly, the intensity distribution of an x-ray scattering experiment is plotted in reciprocal space, i.e., the space spanned by the momentum transfer between incident radiation with wave vector $\mathbf{k}_{i}$ and scattered radiation with wave vector $\mathbf{k}_{f}: \mathbf{Q}=\mathbf{k}_{f}-\mathbf{k}_{i}$ (scattering vector). Here we consider mostly [except for extended x-ray absorption fine structure (EXAFS)] elastic scattering with $\left|\mathbf{k}_{i}\right|=\left|\mathbf{k}_{f}\right|=k=2 \pi / \lambda$, where $\lambda$ is the $\mathrm{x}$-ray wavelength. A sketch of a scattering experiment is shown in Fig. 31. As the penetration depth is considerably smaller than the sample dimensions, only radiation with positive incident and exit angles $\alpha_{i, f}$ can be used in the reflection geometry, the so-called Bragg case. This puts restrictions on the accessible range in reciprocal space, indicated by the three half spheres in the figure. The outer half sphere, with a radius of $2 k$, marks the limit due to the maximum scattering angle of $2 \theta=\pi$ (backscattering). In the Bragg case, only the region between the large half sphere and the two small half spheres is accessible, as for $\mathbf{Q}$ within the latter either $\alpha_{i}$ or $\alpha_{f}$ becomes negative. These regions, the so-called Laue zones, are accessible only in transmission. Note that the orientation of the $x$ axis is 
always along the projection of $\mathbf{k}_{i}$ into the $x y$ plane. A review of especially surface-sensitive $\mathrm{x}$-ray diffraction techniques was presented by Robinson and Tweet (1992). The characterization of self-assembled nanostructures by diffuse x-ray scattering has been reviewed by Schmidbauer, Hanke, and Köhler (2002), focusing on $\mathrm{Ge}$ islands grown by liquid-phase epitaxy and InP islands.

\section{Scattering theory}

The theoretical description of $\mathrm{x}$-ray scattering processes is briefly summarized in the following. The differential cross section of scattering into a certain solid angle $d \Omega$ around $\mathbf{k}_{f}$ is

$$
\frac{d \sigma}{d \Omega}=\frac{1}{16 \pi^{2}}\left|\left\langle\mathbf{k}_{f}|\hat{\mathbf{T}}| \mathbf{k}_{i}\right\rangle\right|^{2} .
$$

This equals the flux of scattered photons into $d \Omega$ for unit flux density of the incoming x-ray beam. In Eq. (7), $\hat{\mathbf{T}}$ $=\hat{\mathbf{V}}+\hat{\mathbf{V}} G_{0} \hat{\mathbf{V}}+\hat{\mathbf{V}} G_{0} \hat{\mathbf{V}} G_{0} \hat{\mathbf{V}}+\cdots$ is the scattering operator,

$$
G_{0}\left(\mathbf{r}-\mathbf{r}^{\prime}\right)=-\frac{1}{4 \pi} \frac{\exp \left(\mathrm{i} k\left|\mathbf{r}-\mathbf{r}^{\prime}\right|\right)}{\left|\mathbf{r}-\mathbf{r}^{\prime}\right|}
$$

is the Green's function of a free particle, and $\hat{\mathbf{V}}$ is the scattering potential, which is derived from the Maxwell equations. We restrict ourselves to the scattering from electron charges (and not from their spins), and, for simplicity, we assume that the $\mathbf{E}$ field is also transversal in the material. The scattering potential is then proportional to the electron density $\rho(\mathbf{r})$ :

$$
\hat{\mathbf{V}}(\mathbf{r})=4 \pi r_{\mathrm{el}} \rho(\mathbf{r})=-k^{2} \chi(\mathbf{r}) .
$$

Here $k=2 \pi / \lambda$ is the wave-vector length in vacuum, $r_{\mathrm{el}}$ $\approx 2.82 \times 10^{-13} \mathrm{~cm}$ is the classical electron radius, and $\chi(\mathbf{r})$ is the crystal polarizability. In a perfect crystal, the polarizability is a periodic function of the coordinates and can be expressed by the Fourier series

$$
\chi(\mathbf{r})=\sum_{\mathbf{g}} \chi_{\mathbf{g}} e^{\mathbf{i g r}}
$$

over the reciprocal-lattice vectors $\mathbf{g}$.

We always deal with ensembles of nanostructures, which introduces statistical fluctuations. As the illuminated area contains many islands and is usually much larger than the coherently illuminated area, the scattered intensity is averaged over a statistical ensemble of island properties, which is denoted by \langle\rangle below.

The scattered intensity is divided into coherent and incoherent, or diffuse, parts. Abbreviating $T_{i f}$ $=\left\langle\mathbf{k}_{f}|\hat{\mathbf{T}}| \mathbf{k}_{i}\right\rangle$, we can write

$$
\begin{aligned}
& I_{\text {total }}=I_{\text {coh }}+I_{\text {diff }}, \\
& I_{\text {coh }} \propto\left|\left\langle T_{i f}\right\rangle\right|^{2},
\end{aligned}
$$

$$
I_{\text {diff }} \propto \operatorname{Cov}\left(T_{i f}, T_{i f}\right),
$$

where $\operatorname{Cov}(a, b)=\left\langle a b^{*}\right\rangle-\langle a\rangle\langle b\rangle^{*}$ is the covariance of random quantities $a, b$. In many cases, the coherent scattering is concentrated in small regions of reciprocal space, e.g., along crystal truncation rods parallel to the sample surface normal. The relevant information about strain and composition is usually contained in the diffuse scattering. Hence we concentrate on the calculation of the latter.

In most cases, the exact solution is difficult or impossible to calculate. On the other hand, calculating the scattered intensity distributions within certain approximations is usually sufficient. Setting $\hat{\mathbf{T}}=\hat{\mathbf{V}}$, one obtains the kinematical approximation, neglecting the effect of scattering on the transmitted wave, which is therefore identical to the incident wave $E_{0}$. Hence extinction and refraction of the primary wave, as well as any multiple scattering processes, are neglected.

The simulations can be improved using the distortedwave Born approximation (DWBA): the scattering potential is divided into two parts $\hat{\mathbf{V}}=\hat{\mathbf{V}}_{A}+\hat{\mathbf{V}}_{B}$ in such a way that for the unperturbed potential $\hat{\mathbf{V}}_{A}$ the exact solution $E_{A}$ of the scattering problem can be obtained. For the perturbation $\hat{\mathbf{V}}_{B}$, which usually contains the scattering potential of the nanostructures, a solution using the kinematical approximation is calculated, with $\hat{\mathbf{T}}=\hat{\mathbf{V}}$. The scattering cross section becomes

$$
\frac{d \sigma}{d \Omega}=\frac{1}{16 \pi^{2}}\left\langle\left|\left\langle E_{A}^{(2)}\left|\hat{\mathbf{V}}_{A}\right| \mathbf{k}_{i}\right\rangle+\left\langle E_{A}^{(2)}\left|\hat{\mathbf{V}}_{B}\right| E_{A}^{(1)}\right\rangle\right|^{2}\right\rangle .
$$

Here $E_{A}^{(1,2)}$ are two independent solutions for the unperturbed potential, where the incident wave belonging to $E_{A}^{(1)}$ is the actual incident wave $\left|\mathbf{k}_{i}\right\rangle$, and the incident wave of the time-reversal of $E_{A}^{(2)}$ corresponds to the scattered wave with wave vector $\mathbf{k}_{f}$. Multiple scattering processes due to $\hat{\mathbf{V}}_{B}$ are neglected, which is justified if the perturbing potential is small compared to $\hat{\mathbf{V}}_{A}$. However, the separation of a particular potential into an unperturbed part and a perturbation is often ambiguous, and the choice usually depends on the scattering geometry. For instance, in grazing-incidence small-angle x-ray scattering or $\mathrm{x}$-ray reflectivity, which are sensitive only to the difference of the refractive index between islands and substrate or matrix material, this difference $\Delta \chi_{0}$ and the shape of all islands $\Omega(\mathbf{r})$ are used in

$$
\hat{\mathbf{V}}_{B}(\mathbf{r})=-k^{2} \Delta \chi_{0} \Omega(\mathbf{r}),
$$

whereas $\hat{\mathbf{V}}_{A}$ contains the polarizability of the substrate material $\chi_{0}$.

For a statistical island distribution, the shape function $\Omega(\mathbf{r})$ is written as a convolution of the statistical island positions with the shape function of an average island. Then, the diffusely scattered intensity in x-ray reflectivity or grazing-incidence small-angle $x$-ray scattering is proportional to the product of the Fourier transforma- 
tion of the averaged shape function $\Omega^{(\text {is) }}(\mathbf{r})$ with the correlation function $C(\mathbf{Q})$ of the island positions

$$
I_{\text {diff }}(\mathbf{Q}) \propto\left|\left[\Omega^{(\text {is })}(\mathbf{Q})\right]^{\mathrm{FT}}\right|^{2} C(\mathbf{Q}),
$$

where ${ }^{\mathrm{FT}}$ denotes the Fourier transformation and the correlation function $C(\mathbf{Q})$ contains the random position of the $m$ th island $\mathbf{R}_{m}$ :

$$
C(\mathbf{Q})=\left\langle\sum_{m, n} e^{-\mathrm{i} \mathbf{Q} \cdot\left(\mathbf{R}_{m}-\mathbf{R}_{n}\right)}\right\rangle .
$$

To consider strain for $\mathrm{x}$-ray and grazing-incidence diffraction with the diffraction vector $\mathbf{g}=\mathbf{h}$, the perturbation potential $\hat{\mathbf{V}}_{B}$ is expressed by

$$
\hat{\mathbf{V}}_{B}(\mathbf{r})=-k^{2}\left[\Delta \chi_{\mathbf{h}} \Omega(\mathbf{r}) e^{-\mathrm{ih} \cdot \mathbf{u}(\mathbf{r})}-\chi^{(\mathrm{m})}\right],
$$

where $\mathbf{u}(\mathbf{r})$ is the displacement field in point $\mathbf{r}$ due to all islands, the polarizability of which differs by $\Delta \chi_{\mathbf{h}}$ from the polarizability $\chi_{\mathbf{h}}^{(\mathrm{m})}$ of the surrounding matrix. For completely uncorrelated islands, the resulting intensity of diffuse scattering is

$$
\begin{aligned}
I_{\text {diff }}(\mathbf{Q}) \propto & N \mid \int d^{3} \mathbf{r} e^{-\mathrm{i} \mathbf{q} r}\left[\chi_{\mathbf{h}}^{(\mathrm{m})}\left(e^{-\mathrm{ihhu}{ }^{(\mathrm{is})}(\mathbf{r})}-1\right)\right. \\
& \left.+\Delta \chi_{\mathbf{h}} \Omega^{(\mathrm{is})}(\mathbf{r}) e^{-\mathrm{ih \mathbf {hu }}{ }^{(\mathrm{is})}(\mathbf{r})}\right]\left.\right|^{2},
\end{aligned}
$$

where $N$ is the total number of irradiated islands and the expression in $\|^{2}$ denotes the island structure factor containing the displacement field $\mathbf{u}^{\text {(is) }}$ around a single island and its shape function $\Omega^{(\mathrm{is})}(\mathbf{r})$. If the island positions are correlated, similar expressions can be found only if one assumes that the deformation fields of different islands do not overlap. The diffusely scattered intensity is then obtained from Eq. (16) by multiplying it by the correlation function according to Eq. (14). If the deformation fields of different islands do overlap, no simple expressions for the diffuse scattering exist. In this case, a possible (not completely exact) solution is to assume perfectly correlated island positions and to convolve the resulting sharp-intensity maxima with a suitably chosen function.

\section{Grazing-incidence small-angle x-ray scattering}

Grazing-incidence small-angle $x$-ray scattering (GISAXS) and x-ray reflectivity (see the following section) are two scattering geometries probing the scattered intensity in the forward direction, i.e., close to the (000) reciprocal-lattice point. Consequently they are not sensitive to the crystalline structure of the samples, but only to the index of refraction $n$, which depends on the electron density averaged over a unit cell. Thus GISAXS and $\mathrm{x}$-ray reflectivity are well suited for the investigation of the shape and the correlation of positions of selfassembled nanostructures. In both techniques, the x-ray beam falls onto the substrate under a small angle, typically several tenths of a degree up to several degrees, and is partly specularly and partly diffusely reflected from the surface and internal interfaces: as the refractive

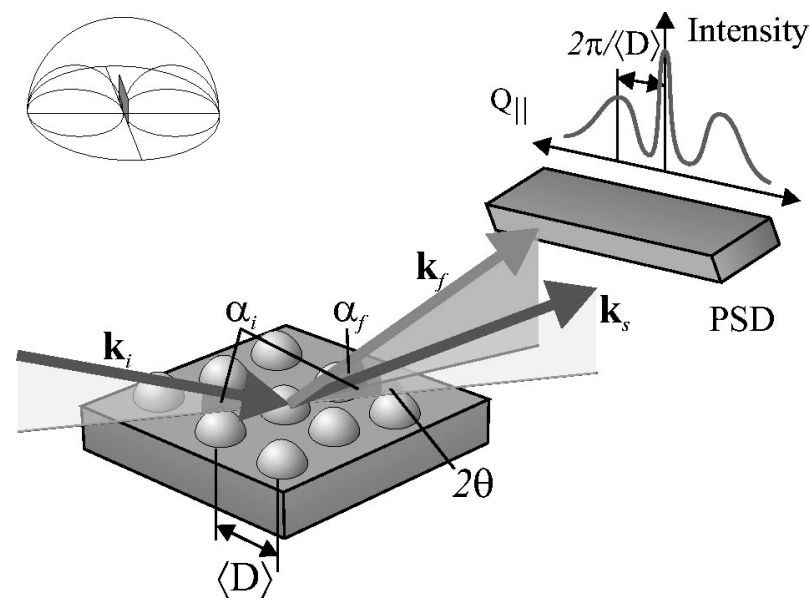

FIG. 32. Scattering geometry in grazing-incidence small-angle $\mathrm{x}$-ray scattering (GISAXS): $\mathbf{k}_{i}$ is the incident beam with an angle $\alpha_{i}$ with respect to the sample surface, $\mathbf{k}_{f}$ is a (diffusely) scattered beam in the direction to the detector, $\mathbf{k}_{s}$ denotes the specularly reflected beam. The in-plane scattering angle is $2 \theta$ and $\alpha_{f}$ is the exit angle of $\mathbf{k}_{f}$ with respect to the surface. Note that $\mathbf{k}_{f}$ does not lie in the plane spanned by $\mathbf{k}_{i}, \mathbf{k}_{s}$, and the surface normal. The upper left inset depicts the accessible range in reciprocal space.

index for matter is slightly smaller than unity $(1-n$ $\simeq 10^{-5}$ typically), total external reflection occurs for incidence angles below the critical angle, which is in the range of typically $0.1^{\circ}-0.5^{\circ}$, depending on material and $\mathrm{x}$-ray energy. Surface roughness or corrugations, such as self-assembled islands, lead to diffuse scattering beside the specularly reflected beam. If the detector is placed at higher or lower exit angle $\alpha_{f}$ than the incidence angle $\alpha_{i}$, but within the same plane as the incident beam and the sample's surface normal x-ray reflectivity, a very high resolution in reciprocal space in the direction parallel to the sample surface is obtained. However, only a limited range of in-plane momentum transfer $Q_{\|}$is accessible due to the Laue zones (see Fig. 31). In GISAXS, by contrast, the detector is rotated out of the plane of the incident beam and surface normal (see Fig. 32). Thus large values of $Q_{\|}$can be reached independent of $\alpha_{i}$ and $\alpha_{f}$. The tradeoff is a lower resolution along $Q_{\|}$than can be obtained with x-ray reflectivity.

For the investigation of self-assembled islands, measurements up to high in-plane momentum transfer are required. In addition, for the measurement of laterally correlated islands, a lateral momentum-transfer range sufficient to evaluate the envelopes of satellite peaks is essential. Schmidbauer et al. (1998) investigated the correlation in single layers of $\mathrm{SiGe}$ islands grown by liquidphase epitaxy using GISAXS and atomic force microscopy. At low densities, islands tended to form dimers or clusters, where islands were aligned along $\langle 100\rangle$ directions. At higher densities, islands arranged more regularly, forming a strongly distorted 2D array (Fig. 33). In addition to the position correlations, the island shape of pyramids with $\{111\}$ side facets and a (001) top facet can also be seen in GISAXS: streaks perpendicular to each 


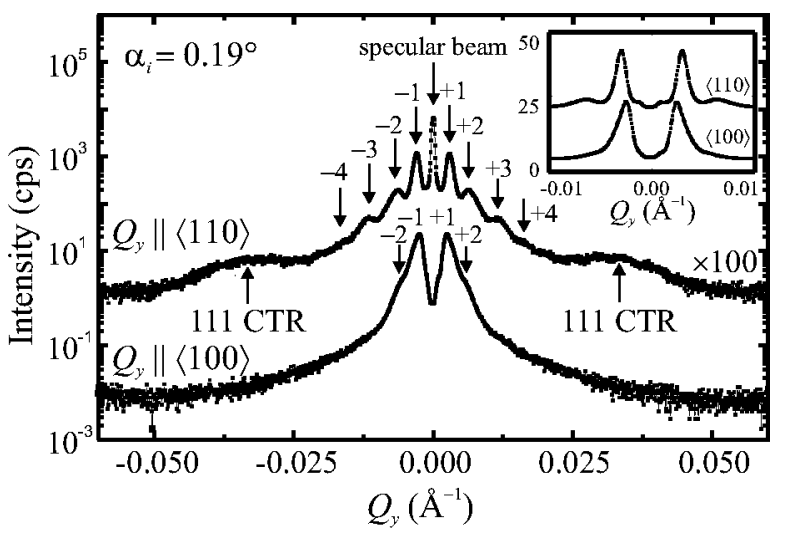

FIG. 33. GISAXS intensity distribution for two different sample orientations. Satellite peaks due to the positional correlation are visible as well as facet streaks due to island shape. From Schmidbauer et al., 1998.

facet appear in reciprocal space, and from the direction of these streaks, the facet angles can be determined, whereas the width of a streak is inversely proportional to the lateral size of the corresponding facet. The same method was applied to InAs islands on GaAs by Zhang et al. (2000), who found a slightly anisotropic array of island positions. $\mathrm{SiGeC}$ islands grown on $\mathrm{Si}(001)$ by $\mathrm{MBE}$ at $600^{\circ} \mathrm{C}$ show a different structure. Stangl, Holý, et al. (1999) investigated a multilayer with 50 periods, in which 0.2 ML's of $\mathrm{C}$ were deposited in each period, followed by $2.4 \mathrm{ML}$ 's of Ge and a spacer of 9.6-nm $\mathrm{Si}$ GISAXS scans revealed the absence of vertical island correlations, nor was any regular lateral arrangement observed but rather a 2D-gas-like distribution was seen, with a preferred lateral distance between the islands and a depletion region around each island, where no other islands nucleated.

DWBA was used for the analysis of GISAXS data by Rauscher et al. (1999), who accounted for the reflection of the primary wave at the sample surface between the islands in the eigensolutions $E_{A}^{(1,2)}$ of the unperturbed scattering potential $\hat{\mathbf{V}}_{A}$ :

$$
E_{A}^{(1,2)}= \begin{cases}e^{i \mathbf{k}_{i}^{(1,2)} \mathbf{r}}+r^{(1,2)} e^{i \mathbf{k}_{R}^{(1,2)} \mathbf{r}} & \text { for } z>0 \\ t^{(1,2)} e^{i \mathbf{k}_{T}^{(1,2)} \mathbf{r}} & \text { for } z<0,\end{cases}
$$

where $\mathbf{k}_{R, T}$ were the wave vectors of the reflected and transmitted waves with relative amplitudes $r$ and $t$, respectively and the sample surface was $z=0$. For islands on a sample surface $(z>0)$, both eigensolutions $E_{A}^{(1,2)}$ had two components, and consequently four scattering processes had to be considered: scattering of the primary beam in the island; reflection of the primary beam and scattering of the reflected beam; scattering of the primary beam and reflection of the scattered beam; and finally scattering of the reflected beam and reflection of the scattered beam. Using this approach, Rauscher et al. studied the structure of SiGe islands grown on $\mathrm{Si}(111)$ with the shape of a triangular pyramid. Figure 34 shows an experimental curve together with simulations in the

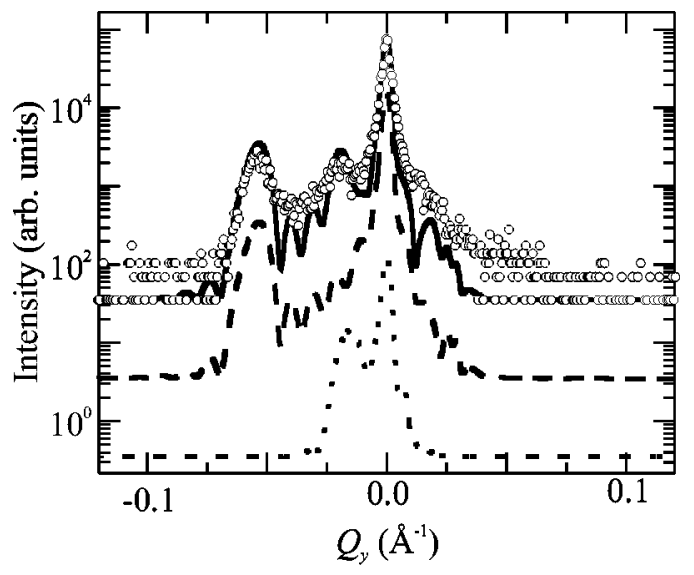

FIG. 34. GISAXS spectrum of a sample with triangular Ge pyramids on a $\mathrm{Si}(111)$ surface: dots; -, kinematical approximation; solid line, full DWBA simulation; dotted line, contribution to the DWBA from the wave reflected at the sample surface prior to scattering in the islands. From Rauscher et al., 1999.

kinematical approximation, i.e., taking only the first terms on the right-hand side of Eq. (17) into account (dashed line) and using the full DWBA (solid line). In the former case, only scattering of the incident wave is considered, and the side maximum at about $q_{y}$ $=-0.02 \AA^{-1}$ cannot be reproduced. In DWBA, the reflection processes at the surface are also taken into account. Of these three processes, the scattering of the reflected beam has a considerable amplitude (dotted line). The asymmetry of the GISAXS spectrum is due to the asymmetric form factor of the triangular pyramids for the chosen [110] sample azimuth.

Simulations based on the DWBA have also been used to investigate the shape and position correlation of $\mathrm{SiGe}$ islands in a $\mathrm{Si} / \mathrm{SiGe}$ multilayer on $\mathrm{Si}(001)$ by $\mathrm{Stangl}$, Holý, et al. (2000). In this case, step bunching and island formation occurred at the same time, and it was expected that the island shape and positions would be influenced by the regular terrace pattern due to the step bunches. In order to distinguish the contribution of the position correlation function $C(\mathbf{Q})$ from that of the shape function $\Omega^{\text {(is) }}(\mathbf{r})$, a Fourier filtering method was used. From a 2D intensity distribution measured in a plane parallel to the sample surface [Fig. 35(a)] the Fourier transformation $J\left(x, y ; Q_{z}\right)$ $=\int d Q_{x} d Q_{y} I_{\text {diff }}(\mathbf{Q}) e^{\mathrm{i}\left(Q_{x} x+Q_{y} y\right)}$ was calculated, as shown in Fig. 35(b). If one assumes that the mean island size is smaller than the mean distance between islands, the central peak of this function depends mainly on $\Omega^{\text {(is) }}(\mathbf{r})$ [Fig. 35(c)], while the more distant parts of this function give information about the correlation properties of the island positions [Fig. 35(d)]. Anisotropies in both island shape and in-plane position correlation were indeed detected, and especially the mutual positions of the islands were considerably more regular in the direction of step bunching. 

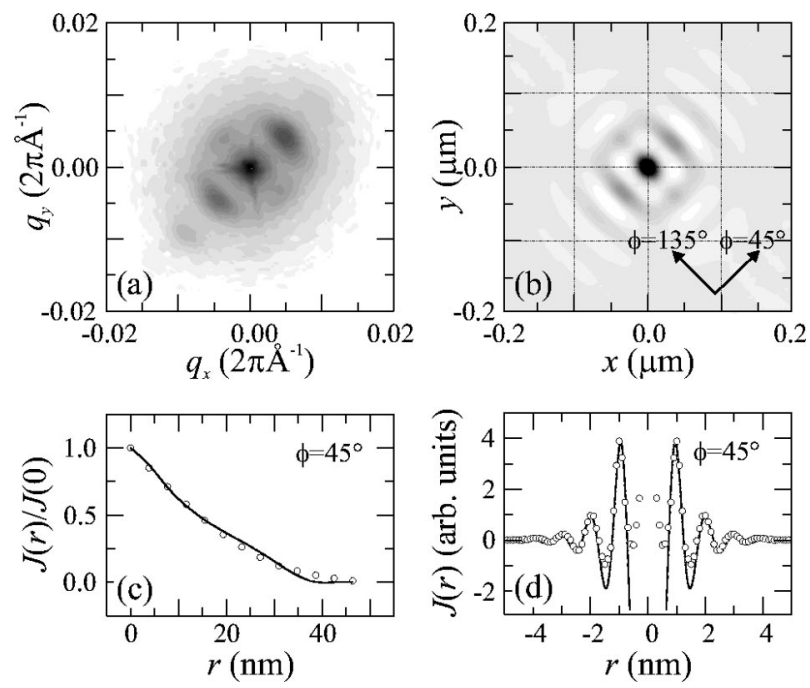

FIG. 35. Lateral island correlation: (a) in-plane GISAXS intensity distribution of a $\mathrm{Si} / \mathrm{SiGe}$ island multilayer, recorded at constant $\alpha_{i}=0.69^{\circ}$ and $\alpha_{f}=0.26^{\circ}$, resulting in a penetration depth of about $900 \mathrm{~nm}$, i.e., illuminating all multilayer periods; (b) the Fourier transform of (a), showing different degrees of spatial island order in different azimuths; (c) and (d) line profiles through (b) for small and large distances $r$, respectively. A fit to the former yields information on island size, while the latter reveals positional correlation.

\section{X-ray reflectivity}

Due to its insensitivity to the crystal lattice and strain fields, x-ray reflectivity has gained an important position in the investigation of the morphology of surfaces and interfaces. The scattering geometry is sketched in Fig. 36. In semiconductor heterostructures, interface roughness and its correlation properties have been extensively investigated by $\mathrm{x}$-ray reflectivity (Headrick and Baribeau, 1993; Kondrashkina et al., 1997). It has been shown above that step bunches can be used as templates to nucleate islands more regularly. Monolayer steps also play an important role in the nucleation and evolution of self-assembled islands. Hence the investigation of steps on semiconductor surfaces and interfaces gives important information relevant for island growth. Due to the interactions of steps, anisotropic and rather regular surface undulations have been found, especially in multilayer structures, where step bunches in subsequent interfaces are often correlated. This correlation is manifested in reciprocal space by so-called resonant diffuse scattering sheets [Holý et al. (1998a); see Fig. 37]: generally, nonplanar interfaces give rise to diffuse scattering. If the morphology of several interfaces is correlated, so is the phase relation of x-rays diffusely scattered from different interfaces, and hence resonant enhancement of diffuse scattering due to interference occurs, similar to the appearance of satellite peaks along the coherent truncation rod (specular reflectivity). From the width along $Q_{z}$ of the resonant diffuse scattering sheets, one can quantitatively determine to what extent the morphologies of subsequent interfaces are correlated. The orientation of the sheets yields the correlation direction,

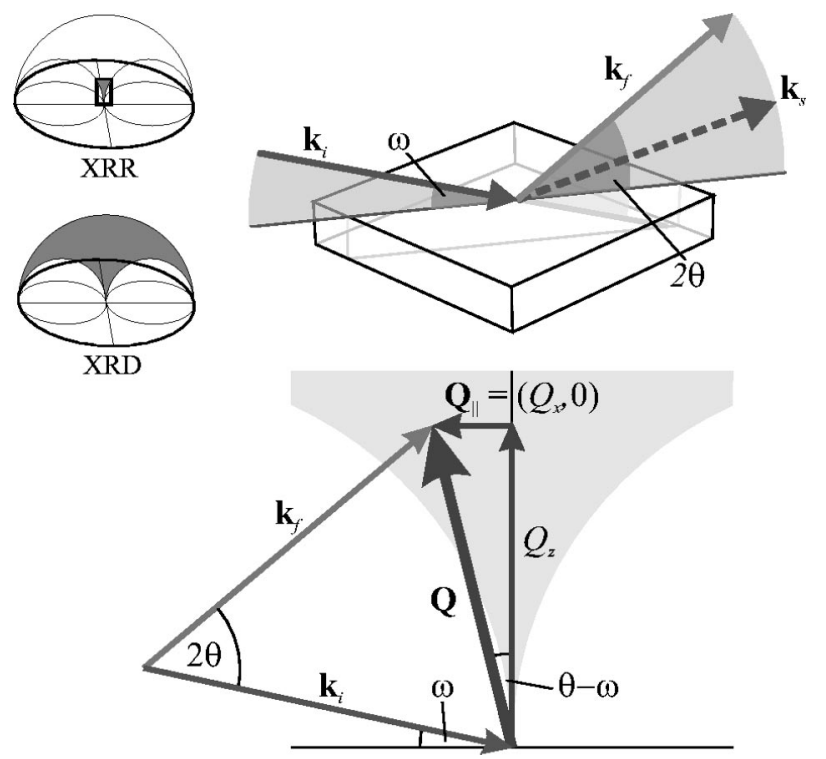

FIG. 36. Scattering geometry in x-ray reflectivity (XRR) in real space (top) and reciprocal space (bottom). $\mathbf{k}_{i}$ is the incident beam with an angle $\omega$ with respect to the sample surface, $\mathbf{k}_{f}$ is a (diffusely) scattered beam in the direction to the detector, $\mathbf{k}_{s}$ denotes the specularly reflected beam. The scattering angle is $2 \theta, \mathbf{k}_{i}, \mathbf{k}_{f}, \mathbf{k}_{s}$, and the surface normal lie in a common plane. The insets at top left depict the accessible range in reciprocal space for XRR and $\mathrm{x}$-ray diffraction (XRD). In XRD the same geometry is used, only the angles $\omega$ and $2 \theta$ are larger. In XRR, instead of $\omega$ and $2 \theta$ often the incidence angle $\alpha_{i}=\omega$ and the exit angle $\alpha_{f}=2 \theta-\omega$ are used in the description.

and the lateral width of the sheets is a measure of the statistical properties of a single interface (autocorrelation). The direction of ripples formed by step bunches is often determined by the miscut direction of the substrate. For particular miscut orientations and growth conditions, however, deviations between ripple direction and miscut direction can be present (Meduña et al., 2001). As Schmidbauer et al. (2001) have pointed out, for a correct determination of the replication direction, reciprocal-space maps have to be recorded in several azimuths.

\section{Coplanar x-ray diffraction}

In x-ray diffraction, unlike GISAXS and x-ray reflectivity, the main contrast is due to strain. The scattering
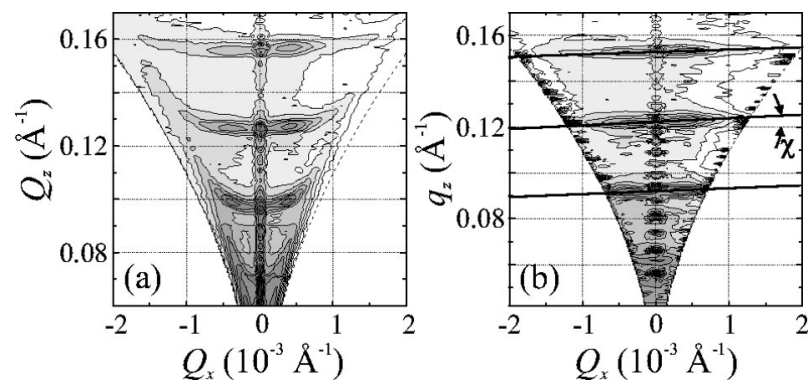

FIG. 37. XRR reciprocal-space map from a rough $\mathrm{Si} / \mathrm{SiGe}$ multilayer exhibiting resonant diffuse scattering sheets: (a) measured; (b) after correction for refraction. From Holý et al., 1998a. 
geometry is the same as in x-ray reflectivity (Fig. 36), but with incident angle $\omega=\alpha_{i}$ and scattering angle $2 \theta=\alpha_{i}$ $+\alpha_{f}$ large, probing the vicinity of reciprocal lattice points $(h k l) \neq(000)$. In self-assembled islands, the lattice strain depends mainly on two factors: the island composition leads to a local deviation of the (bulk) lattice parameter of the island from the substrate material. As the islands are epitaxially coupled to the substrate, both the islands and the surrounding matrix material are additionally strained. Modeling these strain fields, one can extract information on islands from x-ray diffraction data, usually reciprocal-space maps recorded around symmetric and asymmetric Bragg reflections.

The first studies in this area dealt with island multilayers. Due to the partly regular lateral arrangement in $\mathrm{Si} / \mathrm{SiGe}$ islands multilayers, Darhuber et al. (1997) found lateral maxima accompanying the superlattice satellites due to the vertical superlattice structure [a very similar study on InAs/GaAs island superlattices was performed by Faleev et al. (1999)]. From the data, which were recorded using a laboratory $\mathrm{x}$-ray diffractometer, average properties of the islands were obtained. It was demonstrated that the measurements were sensitive to the compressive strain within the islands in regions of reciprocal space with large deviations of the momentum transfer $\mathbf{Q}$ from the reciprocal-lattice point $\mathbf{h}=(h k l)$, while the region with $\mathbf{Q}-\mathbf{h}$ small was sensitive to the tensile strain in the Si matrix between the islands.

From reciprocal-space maps measured using synchrotron radiation, the chemical composition profile in uncapped, single $\mathrm{SiGe}$ island layers on $\mathrm{Si}(001)$ was obtained by Wiebach et al. (2000) and Stangl, Daniel, et al. (2001). In these studies, starting from assumptions based on the island shape from AFM and TEM images, and using different types of Ge profiles along growth direction, the strain distribution was calculated using the finite-element method. The intensity distribution in reciprocal space was simulated using these strain fields and kinematical scattering theory. Refining the shape and the chemical composition, the Ge profile within the islands was determined. For LPE-grown $\mathrm{SiGe}$ islands with a nominal Ge content of $25 \%$, a sharp interface at $1 / 3$ of the island height within the islands with a Ge content of $25 \%$ below and $30 \%$ above was obtained by Wiebach $e t$ al. (2000); see Fig. 38. For samples grown with MBE at $600^{\circ} \mathrm{C}$, it was found that although pure Ge was deposited, pronounced interdiffusion of $\mathrm{Si}$ and $\mathrm{Ge}$ took place during growth, leading to a Ge content increasing from $50 \%$ at the island base to $100 \%$ at the island apex (Stangl, Daniel, et al., 2001).

The effect of capping on island shape and composition was investigated using the same method (Hesse et al., 2002). During capping of dome-shaped SiGe islands at growth temperatures in the range from 550 to $600^{\circ} \mathrm{C}$, additional strong intermixing as well as a considerable flattening and broadening of the islands occurred, as can be seen in Fig. 39. These XRD results agree well with a TEM study of the same capped samples (Fig. 40). While in XRD a laterally constant composition was assumed,

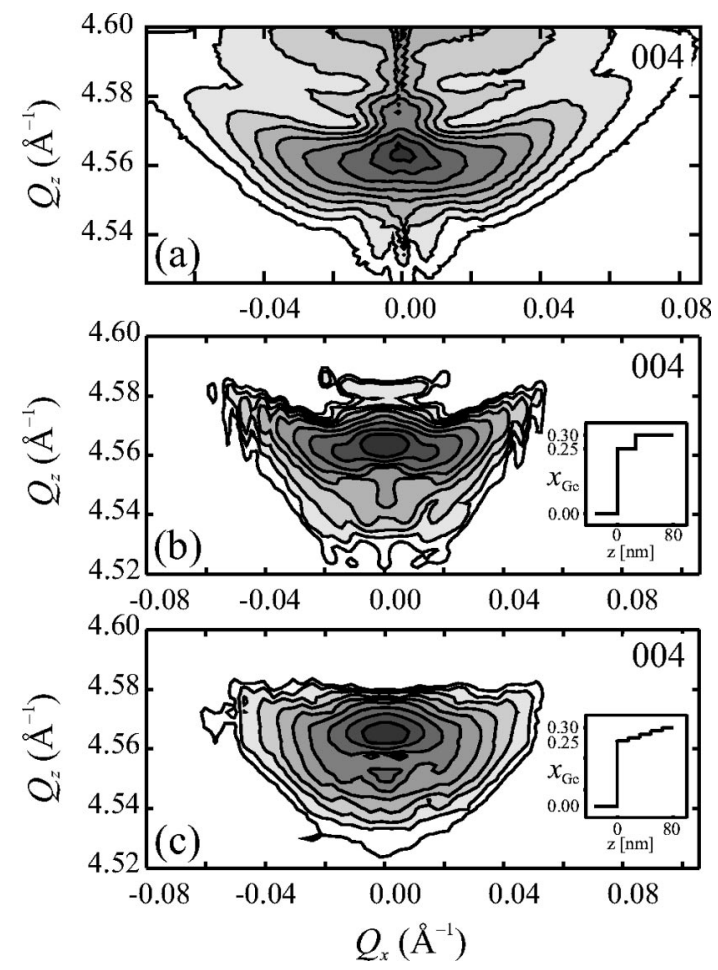

FIG. 38. SiGe island layer grown by liquid-phase epitaxy on $\mathrm{Si}(001)$ : (a) measured (004) reciprocal-space map; (b) and (c) simulations with different assumptions on the vertical Ge distribution. From Wiebach et al., 2000.

the TEM evaluation was performed only for the center of the islands, where the thickness of the specimen was assumed to be smaller than the island width and the projection along the electron beam would not affect the result too much. Intermixing and shape transition can be avoided if capping is performed at lower temperatures of only $460^{\circ} \mathrm{C}$ (Stangl, Hesse, et al., 2003). In addition to the composition, the strain distribution in and around capped islands has been measured. Comparatively large tensile strain values are reached directly beneath and above buried islands. Notably, these regions with large

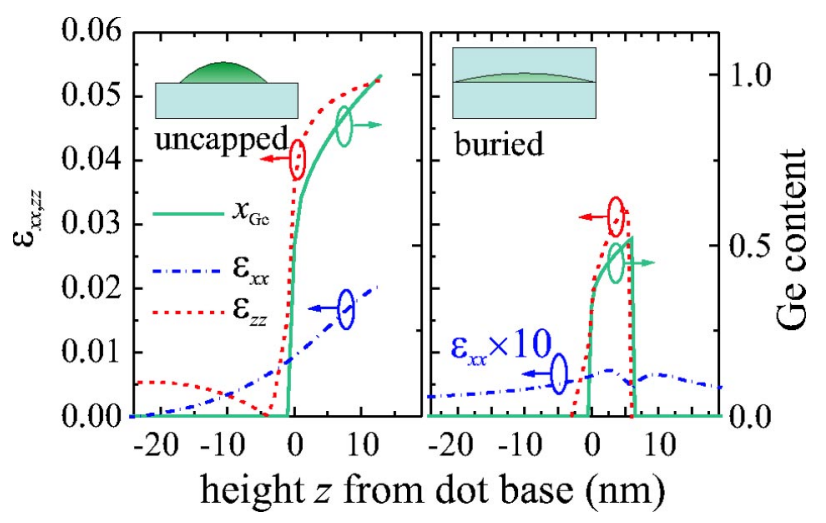

FIG. 39. (Color in online edition) Ge content and strain profile obtained from x-ray diffraction reciprocal-space maps for uncapped dome-shaped islands and after capping with $\mathrm{Si}$ at $T$ $=550^{\circ} \mathrm{C}$. The insets sketch the different island shapes. From Hesse et al., 2002. 


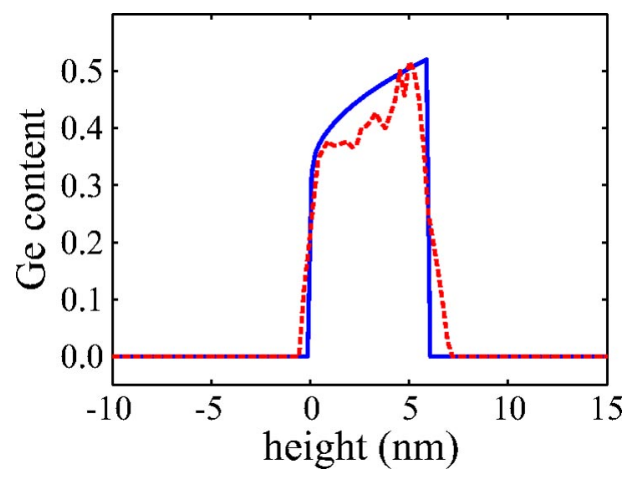

FIG. 40. (Color in online edition) Comparison of Ge contents along island height obtained from x-ray diffraction experiments (solid line, data from Hesse et al., 2002) with results from TEM using digital analysis of lattice images obtained for the island center (dashed line, data from Schmidt, Denker, et al., 2002).

strain can be achieved without incorporation of defects. For uncapped islands with a narrow size distribution, their shape can be immediately deduced from reciprocal-space maps. In this case, the Fourier transformation of shape function $\Omega^{(i s)}(\mathbf{r})$ exhibits narrow streaks, known as facet truncation rods in reciprocal space perpendicular to the island facets. The average strain in the islands can be deduced from the shift of the intensity pattern with respect to the substrate maximum. This method was used by Kovats et al. (2000) for SiGe islands on $\mathrm{Si}(001)$ substrates with a miscut of $0.5^{\circ}$. A complete strain relaxation along the miscut direction was found, but only partial strain relief in other directions.

The 2D or 3D arrangement of islands can be seen directly from reciprocal-space maps. Figure 41 shows reciprocal-space maps of three types of $\mathrm{PbSe}$ island stacking in strain-symmetrized $\mathrm{PbSe} / \mathrm{PbEuTe}$ superlattices grown on $\mathrm{PbTe}(111)$ virtual substrates. The intensity maxima stemming from the position correlations are clearly visible. For PbEuTe spacers below $40 \mathrm{~nm}$, the islands are arranged vertically, hence the diffracted in-

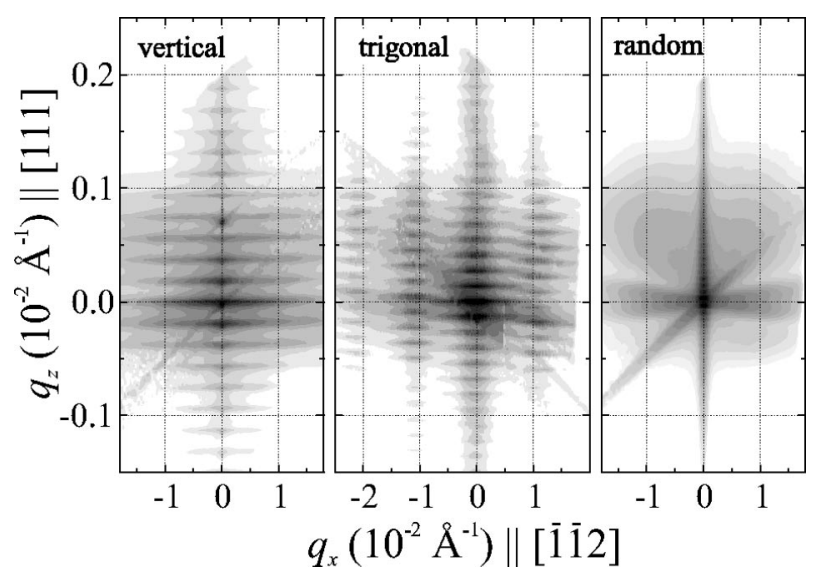

FIG. 41. Reciprocal-space maps (symmetric diffraction 111) measured for $\mathrm{PbSe} / \mathrm{PbEuTe}$ multilayers with three different arrangements of PbSe islands. From Holý et al., 2001. tensity is concentrated in sheets in reciprocal space parallel to the sample surface. For intermediate spacers between 40 and $60 \mathrm{~nm}$, the islands create a highly periodic trigonal lattice and the diffracted intensity exhibits sharp maxima, corresponding to the reciprocal lattice of the trigonal island arrangement. If the primary $\mathrm{x}$-ray beam is well collimated both in the scattering plane and in the direction perpendicular to it, the intensity maxima are cross sections of the reciprocal island lattice with the scattering plane. If the primary beam is not collimated in the direction perpendicular to the scattering plane (as is the case in the usual laboratory setup), the intensity maxima are determined by projections of the reciprocallattice points onto the scattering plane. Finally, for thicker spacers, the island positions are completely random, and the diffracted intensity is proportional to the intensity scattered from a single island.

\section{Grazing-incidence x-ray diffraction}

A frequent problem in the analysis of diffuse signals is that they often are rather weak due to the very small scattering volumes. A single island layer is typically about 5-10 nm thick, whereas the penetration depth of $\mathrm{x}$ rays in XRD is about $10-100 \mu \mathrm{m}$. Hence diffuse scattering from defects in the substrate and from thermal diffuse scattering can outweigh the signal from the islands. A way to circumvent this problem is to measure at very small incidence and exit angles to reduce the penetration depth, as in x-ray reflectivity and GISAXS. In the coplanar XRD geometry, $\alpha_{i}$ and $\alpha_{f}$ determine the incidence angle and the scattering angle, so $\alpha_{i, f}$ cannot be chosen arbitrarily. Therefore diffraction in grazingincidence diffraction is non coplanar, in a plane parallel to the sample surface. With $\alpha_{i, f}$ small, $Q_{z}$ is close to zero, so that grazing-incidence diffraction is sensitive only to the in-plane lattice parameter, and not to lattice parameters and strains in the growth direction. The scattering geometry is very similar to that in GISAXS (see Fig. 32), with two differences: (i) the in-plane scattering angle $2 \theta$ is not small, and (ii) the angle of the incident beam with respect to the crystal planes perpendicular to the surface $\omega$ is important, i.e., the crystal has to be rotated into diffraction condition. Hence grazing-incidence diffraction combines diffraction at lattice planes perpendicular to the sample surface with reflection of the incoming beam from the surface.

The improved signal from the surface layer compared to that from the substrate in grazing-incidence diffraction is due not to an enhancement of the former, but to a reduction of the latter, as most of the x-ray beam is specularly reflected at the surface. Hence for most grazing-incidence diffraction experiments, synchrotron radiation needs to be used. One of the first measurements on self-assembled islands was performed by Williams et al. (1991). They recorded so-called radial scans, i.e., scans along the scattering vector $\mathbf{Q}$, in situ during deposition of $\mathrm{Ge}$ onto $\mathrm{Si}(001)$. Initially, Ge and $\mathrm{Si}$ had the same in-plane lattice parameter, as Ge grew pseudomorphically. Hence the scattering from $\mathrm{Ge}$ and $\mathrm{Si}$ ap- 
peared at the same in-plane momentum transfer in grazing-incidenced diffraction. As soon as roughening was observed at a coverage of 3 ML's (the thickness where hut clusters started to form) in the growing Ge layer, the scan exhibited a shoulder of the Si peak towards larger lattice parameters, and with increasing $\mathrm{Ge}$ layer thickness a distinct second peak at a larger lattice parameter was observed. Thus in this study the relaxation of small, three-dimensional islands could be linked to the roughening of the growth surface, supporting models that assumed that this relaxation is exactly the driving force for roughening and for the formation of small 3D islands.

Of course, not only the strain, but also the shape of islands influences the intensity distribution in grazingincidence diffraction. If, for instance, hut clusters with facets are grown, streaks from these facets can be observed as well (Steinfort et al., 1996). The roughness of buried interfaces can also be accessed, as was shown by Stepanov et al. (1996), who developed a dynamical scattering theory for unperturbed multilayers valid in the grazing-incidence diffraction geometry and treated roughness in the framework of the DWBA. In contrast to GISAXS and x-ray reflectivity, grazing-incidence diffraction measures the crystalline lattice roughness rather than morphological roughness alone, as it is sensitive to contrast in $\chi_{\mathbf{h}}$ instead of $\chi_{0}$, but the general features of diffuse scattering remain the same.

For laterally uncorrelated but extremely sizehomogeneous SiGe islands grown by liquid-phase epitaxy, Grigoriev et al. (2003) used intensity fringes of diffuse scattering around a (220) Bragg reflection to map out both the composition and the strain gradient. The analysis was based on a DWBA calculation of the scattered intensity and finite-element simulations of the strain fields.

The combination of in-plane diffraction and vertical reflection of the incident beam in grazing-incidence diffraction enables a semidirect analysis of diffuse scattering from islands, called "isostrain scattering" by Kegel et al. (2000, 2001), depicted in Fig. 42. Here, instead of creating a model of a whole island and fitting the diffraction pattern by refining the model parameters, only some assumptions on the island properties were made, and the parameters could be obtained directly from the measured intensity distribution. This method is suited for uncapped islands with a sufficient lattice mismatch. Then, the lattice relaxation with respect to the substrate is a function of height above the surface, and a slice through an island at this height has a certain lateral width. In reciprocal space, this slice gives rise to diffuse scattering with a width inversely proportional to the slice size, centered around the reciprocal-lattice point corresponding to the relaxed lattice. Hence a measurement exhibits an intensity distribution spread along the diffraction vector $\mathbf{h}$ (the "radial" direction $Q_{r}$ ) from the reciprocal-lattice point of the substrate to that of the most relaxed part at the island top. In the angular direction $Q_{a}$ perpendicular to $\mathbf{h}$, the width of the intensity distribution yields information on the width of the slice as a function of $\mathbf{h}$, i.e., as a function of lattice parameter. The height of the slice above the surface can be obtained as well: due to the interference between beams scattered within the island and reflected at the sample surface before and/or after scattering [see Rauscher et al. (1999) and Sec. III.E], the intensity distribution along $Q_{z}$ exhibits a characteristic maximum, from which the height of the slice can be obtained. Additionally, the curvature of the slice can be measured from the width of the diffuse scattering along $Q_{a}$ as a function of $Q_{z}$ for constant $Q_{r}$, so that finally a complete picture of the lattice parameter distribution within the island is obtained. Furthermore, measuring radial scans for a weak and a strong reflection, such as (200) and (400), yields information on the chemical composition as a function of lattice parameter: for InGaAs, the structure factor of weak reflections depends sensitively on the In content. Thus, for III-V compounds, the isostrain scattering method virtually decomposes an island into curved slices with a certain width, a certain composition, a certain height above the sample surface, and a certain strain (Fig. 43).

Several restrictions have to be considered: for systems with a small lattice mismatch, the intensity might not be spread out enough along $Q_{r}$ to allow for a separation of isostrain sheets. If the strain increases sufficiently fast with height, however, if connot easily be obtained from the scattered intensity pattern itself: an isostrain analysis is still possible, but the resulting error bars can only be determined a posteriori. Moreover, the range in $Q_{r}$ for which $Q_{r}$ can be related to a certain strain is not directly evident from the measurements. Near the substrate reflection, diffuse scattering from the substrate usually blurs the intensity scattered from the islands, so that the data for very small strain values (typically below $0.5 \%$ ) are usually not very reliable. Hence the analysis works much better for InGaAs islands on GaAs (7\% maximum lattice mismatch) than for $\mathrm{SiGe}$ islands on $\mathrm{Si}(4 \%$ maximum lattice mismatch).

The upper limit of $\left|Q_{r}-h_{r}\right|$ is also not straightforward to determine from the measurement. For SiGe islands on $\mathrm{Si}$, the data evaluation has not yielded consistent results when the analysis was performed for a region too far from the substrate reflection, which can be used to determine the cutoff in $Q_{r}$. Alternatively, a comparison of the obtained isostrain slice height with the total island height, e.g., from AFM, can serve to determine this cutoff. For buried samples, a determination of the vertical strain variation is not possible, because the interference of beams reflected at the sample surface is required. For $\mathrm{SiGe}$, as one important example, no weak reflections exist for the analysis of chemical composition (however, anomalous scattering may be used to replace weak reflections; see below). The experiments mentioned above deal with a random distribution of island positions, so that the obtained diffuse scattering is equivalent to that from a single island, multiplied by the number of illuminated islands. Positional correlations of islands have been observed in grazing-incidence diffraction as well. 


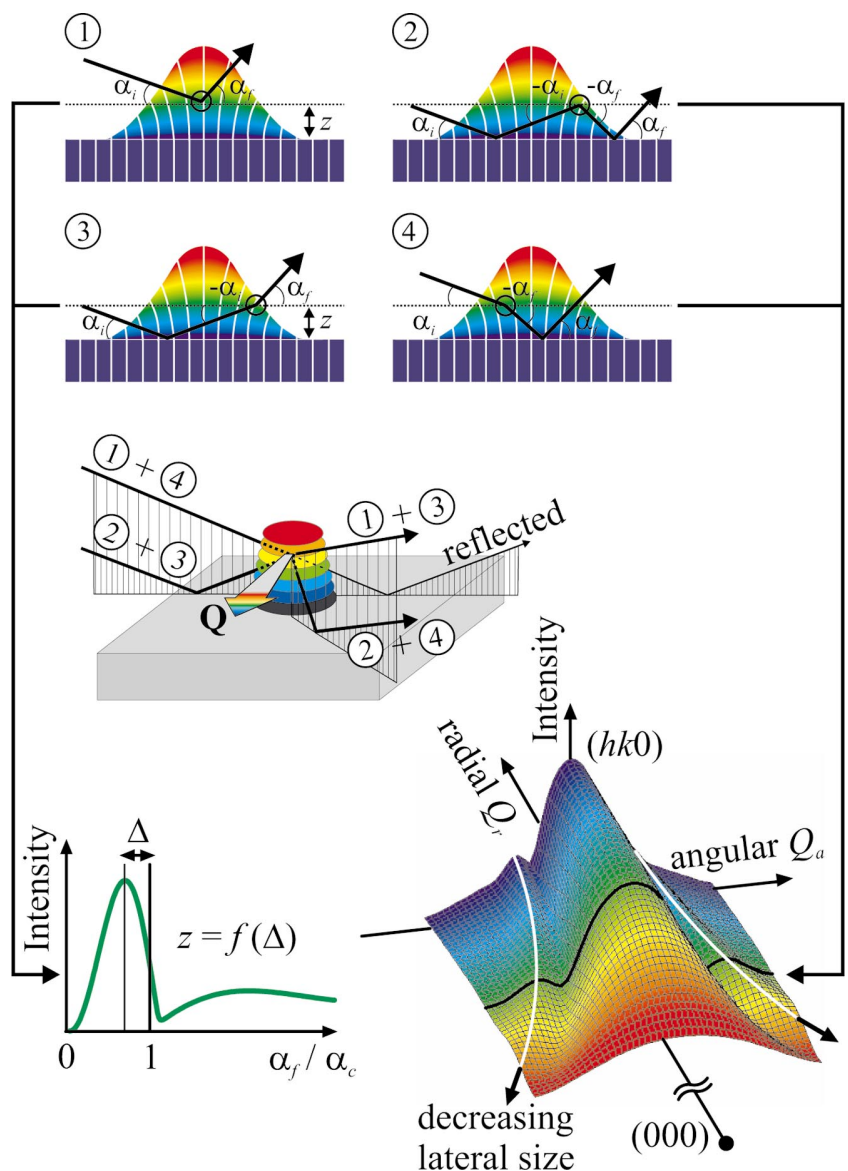

FIG. 42. (Color) Four scattering processes from first-order perturbation theory sketched in side view (top panels) as well as in $3 \mathrm{D}$ (center panel). The resulting intensity distribution is sketched in the lower panels. The interference of beams of the four scattering paths leads to a characteristic shift of the primary maximum along $Q_{z}$ for each isostrain volume at a certain height above the substrate surface (left); with increasing distance from the reciprocal lattice point of the substrate along $Q_{r}$, the width of the intensity distribution along $Q_{a}$ increases (right). From Kegel et al., 2000, 2001.

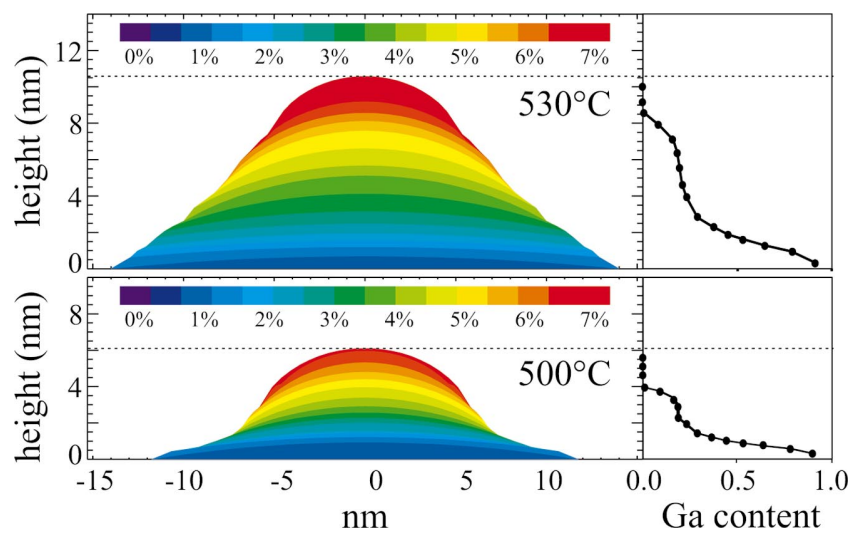

FIG. 43. (Color) In-plane strain with respect to the substrate (left panels) and Ga content in the center of InGaAs islands as obtained from the isostrain-scattering method, for islands grown at two different temperatures. From Kegel et al., 2001.
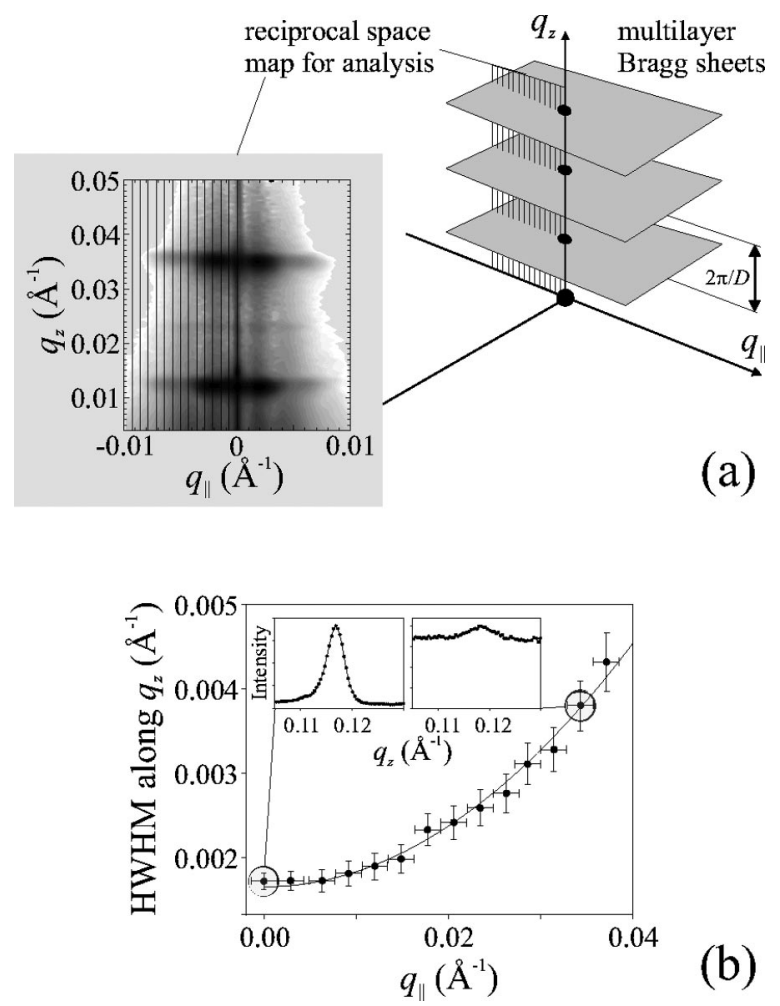

(b)

FIG. 44. Vertical island correlation: (a) sketch of the diffuse scattering sheets in grazing-incidence diffraction due to a $\mathrm{SiGe}$ island multilayer with vertically correlated island positions, together with a measured intensity distribution; (b) increase of the half-width along $q_{z}$ as a function of lateral momentum transfer $q_{\|}$[relative to the (220) Bragg point] of a resonant diffuse scattering sheet due to the imperfect vertical stacking of SiGe islands. From Kegel et al., 1999b.

Although during growth the correlations evolve mostly due to strain fields, here strain itself is not the focus of the measurements, but is only used as a marker, producing contrast with the same spatial arrangement as the islands. The lateral correlation of islands has been investigated by Kegel et al. (1999a) for a Ge/Si superlattice. Grazing-incidence diffraction measurements using an analyzer crystal in the diffraction plane in order to enhance the resolution revealed lateral satellites. It turned out that both the shape of the islands and the positional correlation had to be taken into account in order to correctly interpret the measured intensity distribution. For islands arranged in disordered columns in multilayers, resonant diffuse scattering sheets parallel to the sample surface could be observed [see Fig. 44(a)]. The width of these sheets increases as a function of the in-plane momentum transfer $Q_{\|}$[Fig. 44(b); more precisely the deviation of $Q_{\|}$from the nearest reciprocal lattice point; see Kegel et al. (1999b)], and the rate of increase is a measure of the disorder in the column. Figure 45 shows the degree of correlation $P$ as a function of spacer thickness. Solid squares denote results from x-ray diffraction, which are compared to those obtained from a TEM study (open squares; from Kienzle et al., 1999). Differences in the statistics can be measured using x-ray dif- 


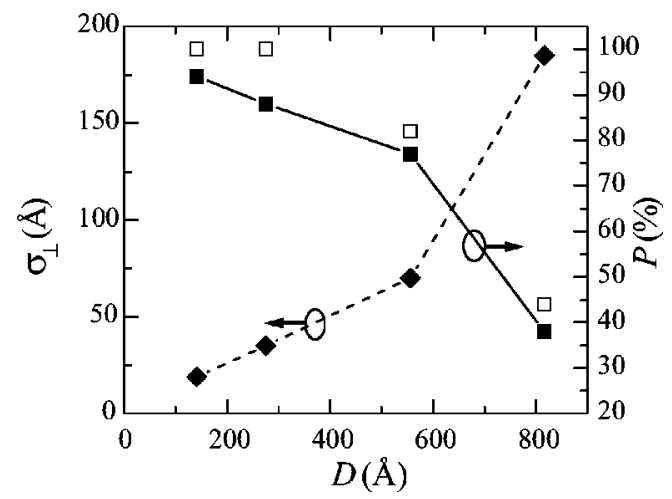

FIG. 45. Decrease of the degree of vertical correlation $P$ of island positions in a $\mathrm{Si} / \mathrm{SiGe}$ multilayer as a function of $\mathrm{Si}$ spacer thickness, calculated from the rms deviation of island positions in subsequent layers $\sigma_{\perp}$. The solid symbols are results from a grazing-incidence diffraction experiment, open symbols are results from TEM. From Stangl, Roch, et al., 2000.

fraction, even in a range where TEM shows only perfect correlation (Stangl, Roch, et al., 2000).

\section{Anomalous x-ray scattering}

In anomalous diffraction, a diffraction curve is recorded at different energies in the vicinity of an absorption edge of one of the sample's constituent elements. The scattering power of this element changes for the two energies, while all other details of the experimental setup remain unchanged: for a small energy variation, the scattering power of all other elements, which do not exhibit absorption edges near the employed energies, remains practically constant, and small changes in scattering angles have virtually no influence on, e.g., the illuminated sample area. Hence the composition of the sample can be obtained from the ratio of intensities measured at different energies.

As anomalous scattering is a way of determining the content directly from an intensity ratio, without elaborate model fitting, it is desirable to combine it with a method to achieve spatial resolution directly as well.

For InAs islands on GaAs, anomalous scattering was employed for the determination of composition by Schuelli et al. (2002). Anomalous diffraction replaced the measurement at weak and strong reflections of GaAs.

For $\mathrm{Ge}$ islands on $\mathrm{Si}$, no weak reflections exist and anomalous scattering provides the possibility of also using isostrain scattering for this material system. Such studies have been performed by Magalhaes-Paniago et al. (2002) and Schülli et al. (2003). While the former group investigated the anomalous effect at the (220) inplane Bragg reflection, the latter used high-index reflections up to (800) in order to considerably enhance the sensitivity of the experiment (see Fig. 46): The maximum change in the scattering strength of $\mathrm{Ge}$ at the $K$ edge at about $11105 \mathrm{eV}$ is only about $20 \%$ for $\mathbf{Q}=0$. Thus for alloyed islands the changes are quite comparable to typical error bars. At high momentum transfers, however, the ratio increases, and the maximum change at the

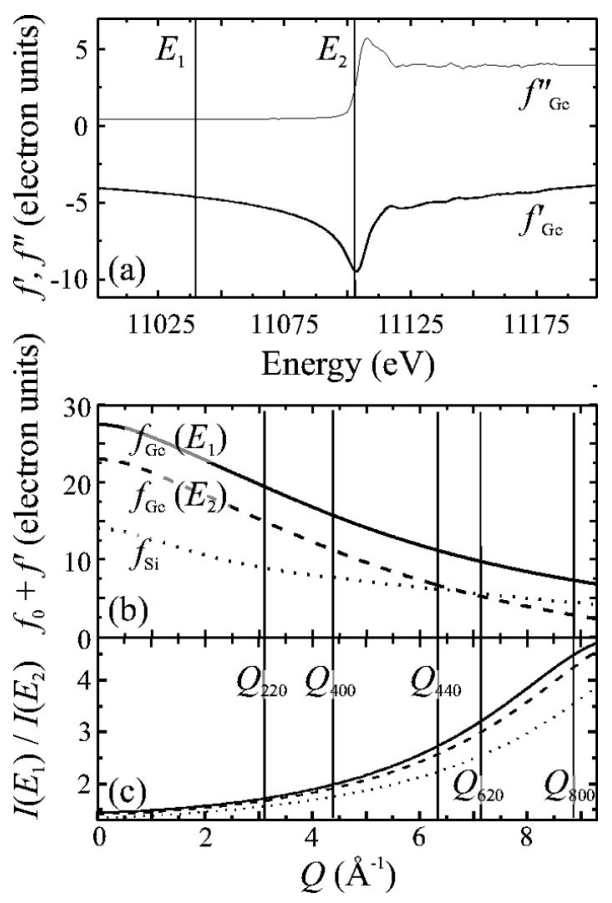

FIG. 46. Anomalous x-ray scattering: (a) anomalous corrections to the atomic scattering factor $f^{\prime}$ and $f^{\prime \prime}$ as a function of energy around the Ge $K$ edge; (b) momentum-transfer dependence of the real part of the atomic scattering factor for Si and for Ge for two energies indicated in (a); (c) intensity ratio for scattering at the two energies-solid line, pure Ge; dashed line, $\mathrm{Si}_{0.1} \mathrm{Ge}_{0.9}$; dotted line, $\mathrm{Si}_{0.3} \mathrm{Ge}_{0.7}$. From Schülli et al., 2003.

(800) reflection is about a factor of 3. For SiGe islands where 7 ML's of Ge had been deposited at $\mathrm{Si}(001)$ at a temperature of $600{ }^{\circ} \mathrm{C}$, Schülli et al. detected a rather abrupt change in the Ge composition from 0 to about $80 \%$ within a height interval of $3 \mathrm{~nm}$. Above this transition the composition remained at about $80 \%$ up to the island top at a height of about $12 \mathrm{~nm}$ (see Fig. 47).

Recently, anomalous scattering in grazing-incidence diffraction geometry has been used by Malachias et al. (2003) for the investigation of lateral inhomogeneities of the chemical composition of uncapped Ge domes grown on $\operatorname{Si}(001)$. Intensity distributions in the horizontal $Q_{r} Q_{a}$ plane were measured integrating over the exit angle $\alpha_{f}$ at energies close to and far from the Ge $K$ absorption edge. The extracted angular scans were sensitive not only to the shape of an isostrain volume, but also to the lateral variation of its composition. The comparison of measured data with kinematical simulations revealed the existence of a Si-rich core around the vertical island axis and close to the substrate interface, and the Ge concentration at the island apex was higher than $70 \%$. This finding differs from the results by Schülli et al. (2003), which might be ascribed to the different growth techniques used.

\section{Coherent diffraction}

So far, model fitting has been used in order to reconstruct the real-space structure from a measured diffrac- 

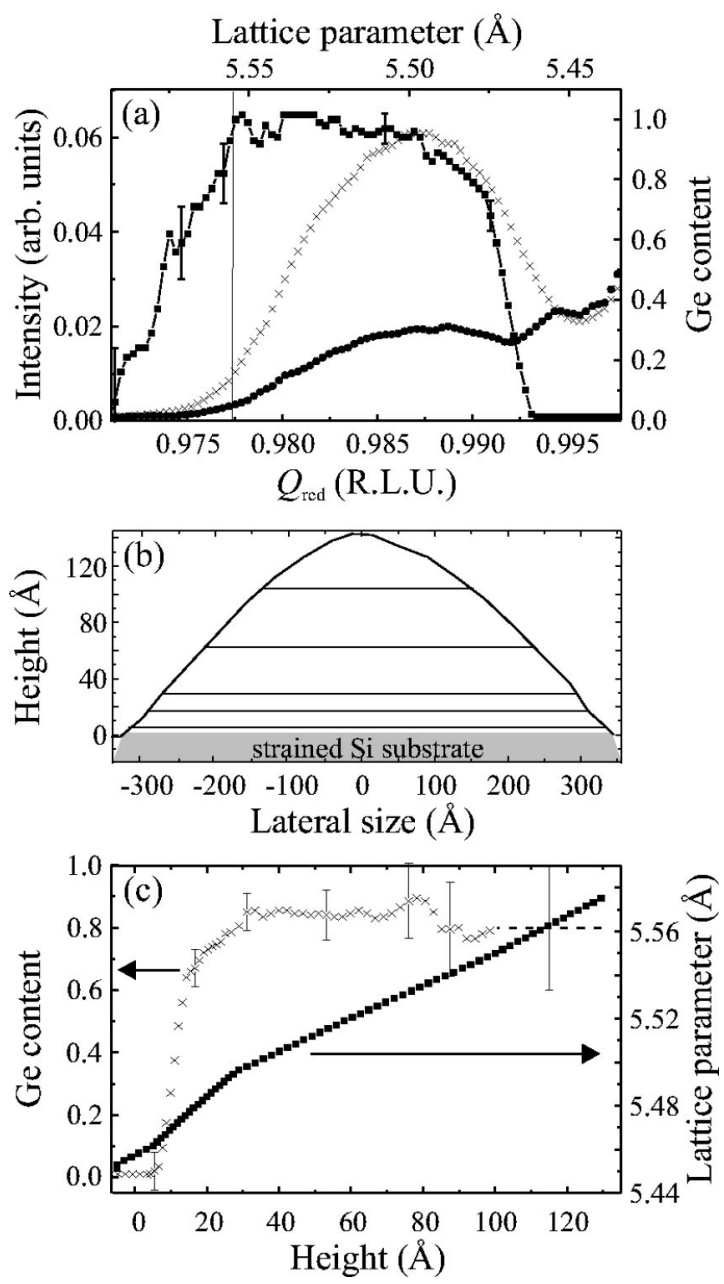

FIG. 47. Anomalous diffraction from SiGe islands: (a) radial scans in grazing-incidence diffraction geometry around the (800) Bragg reflection: $\times$, recorded at $11043 \mathrm{eV}$; $\bullet$, recorded at $11103 \mathrm{eV}$; $\mathbf{\square}$, intensity ratio; (b) lateral dimension; and (c) $\mathrm{Ge}$ content of SiGe islands and lattice parameter as a function of island height, obtained from anomalous $\mathrm{x}$-ray scattering. From Schülli et al., 2003.

tion pattern. This is necessary due to the loss of phase information. There have been attempts, however, to reconstruct the phase in the scattered signal, which in consequence allows for the direct calculation of real-space images. As an example, Robinson et al. (2001) recorded diffraction images of nanoscale gold particles using a coherent x-ray beam: only then is the full information present in the scattered radiation. Assigning phases randomly to points in the measured reciprocal-space map, and applying special filtering techniques and Fourier transformation to real space and back to reciprocal space, Robinson et al. iteratively obtained the actual phases. The size and shape of gold particles have been successfully determined using this method, although the spatial resolution is not yet competitive with AFM, STM, or TEM. So far, only unstrained particles have been investigated; the investigation of islands exhibiting anisotropic strain fields or embedded in a strained matrix is not yet feasible.

\section{Extended x-ray absorption fine structure}

EXAFS is sensitive to the local environment of certain types of atoms due to the interference of an electron removed by the incident $x$ rays from a shell with the electron waves backscattered from the neighboring atoms. The x-ray energy is tuned close to the absorption edge of a certain element, so that predominantly electrons from the particular shell of that element are excited. The interference depends on the excess energy (and thus the wavelength) of the excited electron and the type and distance from the probed atom of the neighboring atoms. Hence it is possible to probe the local environment of that element.

Using the Ge $K$ absorption edge, the local environment of $\mathrm{Ge}$ atoms in SiGe islands on $\mathrm{Si}$ has been investigated. If the islands consisted of pure Ge, only those atoms at the interface to the substrate would have $\mathrm{Si}$ atoms as nearest neighbors. For alloyed islands, a large portion of $\mathrm{Ge}$ atoms would have $\mathrm{Si}$ atoms as neighbors. For $\mathrm{SiGe}$ islands grown by deposition of $\mathrm{Ge}$ on $\mathrm{Si}(001)$ and $\mathrm{Si}(111)$ by chemical vapor deposition at $600^{\circ} \mathrm{C}$, Boscherini et al. (2000) found clear evidence for alloying in x-ray absorption fine structure. For (001) substrates an average $\mathrm{Si}$ content of $30 \%$ within the islands was detected; for (111) substrates the amount of Si was close to $50 \%$. Very similar results for $\mathrm{SiGe}$ islands grown by MBE were reported by Kolobov et al. (2002).

A combination of grazing-incidence diffraction with EXAFS was used by Grenier et al. (2002) and Proietti et al. (2002). This combination makes it possible to determine even the local environment of atoms of a certain type occurring in a region with given strain. Grenier $e t$ al. (2002) applied the method for the determination of local chemical composition for InAs islands on $\operatorname{InP}(001)$.

\section{F. Optical methods}

Studying light scattering from a $\mathrm{Si}_{0.8} \mathrm{Ge}_{0.2}$ film during growth in a MBE chamber, Chason et al. (1998) investigated the size of evolving islands. With a broadband light source the reflectivity was spectroscopically recorded as a function of momentum transfer. This technique is sensitive to length scales from about 120 to $540 \mathrm{~nm}$, which fits the range of island spacings in this study. The sample surface can be considered as an optical grating with a period varying with $\mathrm{Ge}$ coverage. From the onset of islanding on, the island spacings decreased, indicating an increase in island density, up to a coverage of about $4.8 \mathrm{~nm}$ Ge. Above this thickness, the distances increased again due to ripening. Although these investigations do not give very detailed information on island properties without previous knowledge, the technique seems well suited for growth monitoring (Pidduck et al., 1992).

Another method for the in situ study of stress was presented by Floro et al. (1998, 2000). Deflecting a laser beam converted by a Fabry-Perot etalon into an array of parallel beams from the sample, which had the form of a cantilever free to bend, Floro et al. measured the curva- 
ture of the sample via the distance of deflected laser spots. This yielded a quantitative measure of the global epilayer stress, which could be linked to other sample properties. During deposition of $\mathrm{Si}_{0.8} \mathrm{Ge}_{0.2}$, the stress in the growing film increased during growth of the $2 \mathrm{D}$ wetting layer. As soon as hut clusters formed, the stress decreased to an almost constant level. When the film thickness was further increased, another decrease of stress was found during the island's shape transition from hut clusters to domes.

\section{Raman scattering}

Depending on the propagation direction and the polarization of the incident and scattered light, Ramanscattering experiments give access to both optical and acoustic phonon modes. For cubic systems and for equal in-plane strain tensor components $\varepsilon_{x x}=\varepsilon_{y y}$, the strain splits the optical phonons into a singlet and a doublet component (Groenen et al., 1999). If the corresponding phonon deformation potentials $\tilde{K}_{11}$ and $\tilde{K}_{12}$ are known, values for $\varepsilon_{x x}$ and $\varepsilon_{z z}$ can be deduced without any simulations from the relative frequency shifts $\left(\Delta \omega / \omega_{0}\right)_{S}$ and $\left(\Delta \omega / \omega_{0}\right)_{D}$ of the singlet and doublet components.

Groenen et al. (1999) used this method to investigate the average strain in 3-nm-high and 25-nm-wide InAs dots buried in InP. For this material combination the gap between the InAs and the InP optical phonons is particularly wide and hence the spatial confinement of optical phonons inside the quantum dots is large. Due to the rather weak transverse-optical (TO) phonon dispersion, energy shifts due to phonon confinement are negligible. Therefore one can analyze the dot phonon frequencies just by taking into account the strain-induced shifts. For Ge islands on $\mathrm{Si}$, the Raman frequencies $\omega_{\mathrm{SiSi}}$, $\omega_{\mathrm{SiGe}}$, and $\omega_{\mathrm{GeGe}}$ allow for the determination of $\varepsilon_{x x}, \varepsilon_{z z}$, and the composition $x_{\mathrm{Ge}}$ according to Cazayous et al. (2002). Further studies on polarized Raman scattering from Ge domes were performed by Kamenev et al. (2003). Tan et al. (2003) investigated small buried Ge islands ( $2 \mathrm{~nm}$ height, $20 \mathrm{~nm}$ diameter). From the LOTO splitting of the Ge-Ge mode and the frequencies of the Ge-Ge and $\mathrm{Si}-\mathrm{Si}$ modes both the average biaxial strain $(\approx-0.034)$ and the mean Ge content $(\approx 80 \%)$ of the uncorrelated islands in the multilayer were determined.

With a focused laser spot and confocal entrance optics, Raman topography was performed on very large islands (300 nm height, $700 \mathrm{~nm}$ width) using a displacement sample stage to record line scans with a step of $0.1 \mu \mathrm{m}$. The probing depth was about $400 \mathrm{~nm}$. Information on the variation of $\epsilon_{x x}$ and $\epsilon_{z z}$ with height in a single large Ge island was obtained from a comparison with finite-element method simulations.

In quantum-dot multilayers, the spatial island correlation breaks the translational invariance, and acoustic phonons are Raman active, leading to interference effects. Cazayous et al. (2001) have shown that this interference contrast provides information on the degree of

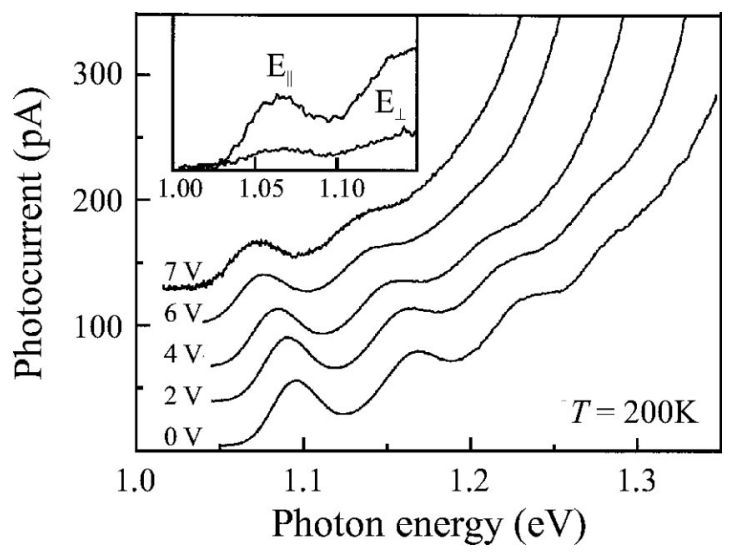

FIG. 48. Photocurrent spectra of InAs islands embedded in a $p-i$ - $n$ structure at different reverse biases, showing a strong quantum confined Stark effect. Peaks due to the interband transitions in the dots are visible. The inset shows the polarization dependence of the $0-\mathrm{V}$ spectrum at $300 \mathrm{~K}\left(E_{\|}\right.$is parallel to the growth plane, $E_{\perp}$ is along the growth direction). From Fry, Itskevich, Mowbray, et al., 2000a.

vertical correlation in $\mathrm{Si} / \mathrm{Ge}$ island superlattices. Raman-scattering experiments on $\mathrm{Si} / \mathrm{SiGe}$ superlattices have been interpreted assuming zone-folding phenomena in the acoustic-phonon dispersion (Tan et al., 2003). Cazayous et al. argue that for a small number of double layers, low-frequency resonant Raman scattering may originate from the 3D charge confinement in the islands. This determines the interference envelopes, whereas the island layer stacking determines the interference period.

\section{Photoluminescence}

Photoluminescence spectroscopy probes energy levels of islands close to the band edges. Such investigations are supplemented by photocurrent and absorption spectroscopy, which access in addition excited states. Depending on the band-edge alignments between the islands and the matrix material, either direct transitions in real space and in $k$ space occur, as for InAs islands embedded in $\mathrm{GaAs}$, or, as in the case of $\mathrm{Si} / \mathrm{SiGe}$ nanostructures, indirect transitions in real space and in $k$ space. Recombining holes are confined in the Ge-rich islands, whereas electrons are confined in the strained Si close to the islands. In the following, we shall restrict ourselves to the discussion of these two model systems, in order to illustrate the importance of structural information for the description of the optical properties of islands.

For pyramidal shaped pureInAs islands, $\mathbf{k} \cdot \mathbf{p}$ calculations (Holm et al., 2002) and first-principles bandstructure calculations have revealed that the hole wave function is confined within the pyramid in a spatial range below that of the electron wave function. This was not confirmed by photocurrent spectroscopy [Barker and O'Reilly (2000); Fry, Itskevich, Mowbray, et al. (2000); Fry, Itskevich, Parnell, et al. (2000); see Fig. 48]. This reversal could only be explained if a compositional gradient, i.e., $\mathrm{Ga}_{1-x} \mathrm{In}_{x}$ As with $x$ increasing from the island bottom to its apex, is assumed. The holes tend to be 


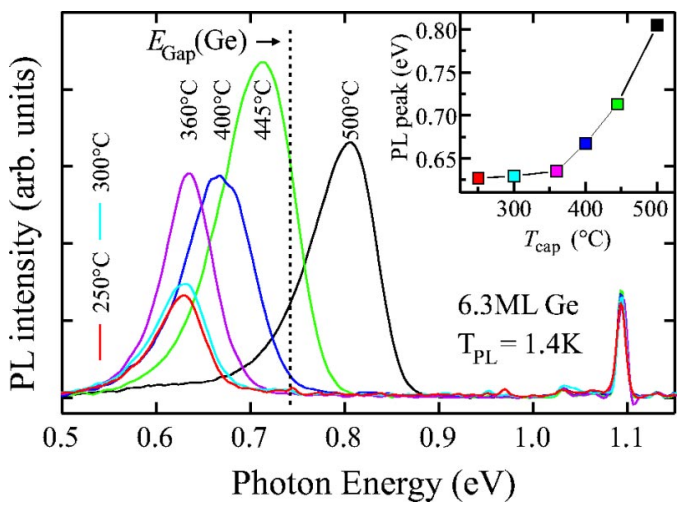

FIG. 49. (Color in online edition) Photoluminescence (PL) spectra from $\mathrm{SiGe}$ hut clusters overgrown at different energies. Inset: the PL line position saturates for $T_{\text {growth }}<360{ }^{\circ} \mathrm{C}$. From Denker et al., 2003b.

localized in the region with the largest In composition, i.e., close to the island apex. Consequently these results imply that the GaInAs islands have a permanent dipole moment due to their asymmetric shape, and its sign is determined by the compositional gradient. These findings are in agreement with other characterization methods like TEM, XRD, and cross-sectional STM.

Ge-related photoluminescence is typically observed from the wetting layer, with rather narrow no-phonon and TO-phonon replica lines, and for Ge coverages above about 5 ML's additionally from the islands, with a rather broad emission at lower energies. Usually, in the emission from the islands the no-phonon and TO-phonon replica cannot be separated from each other due to line broadening caused by island inhomogeneities and the existence of several SiGe alloy phonon modes. For Ge hut cluster islands as well as for domes, capping at temperatures below about $360^{\circ} \mathrm{C}$ ensures negligible $\mathrm{SiGe}$ intermixing. Not only the shape and size, but also the high Ge content of $x=0.9-1.0$ is preserved during capping. Under such conditions, emission from the hut clusters at energies of $0.63 \mathrm{eV}$ was observed by Denker et al. (2003b), more than $110 \mathrm{meV}$ lower than the bandgap energy of unstrained Ge (Fig. 49). Island-related photoluminescence was observed for temperatures up to $185 \mathrm{~K}$. For a base width of $10 \mathrm{~nm}$ and a height of $1.3 \mathrm{~nm}$ of the $\mathrm{Ge}$ islands, assuming that they are completely strained to the Si substrate, and considering the valenceband offset, the heavy hole confinement energy, and the Si band gap, a recombination energy of $0.67 \mathrm{eV}$ results in good agreement with the experimental data. The photoluminescence intensity rolloff with increasing temperature is explained by the escape of weakly bound electrons from the tensile strained $\mathrm{Si}$ with an activation energy of $40 \mathrm{meV}$. The small hut cluster islands confine holes in all three dimensions, with a large confinement energy in the growth direction. The lateral confinement within the Ge dots may be described by a parabolic potential of about 10-20 nm diameter and about $300 \mathrm{meV}$ height. As determined by capacitance spectroscopy (Brunner, 2002), the lateral confinement results in dis-

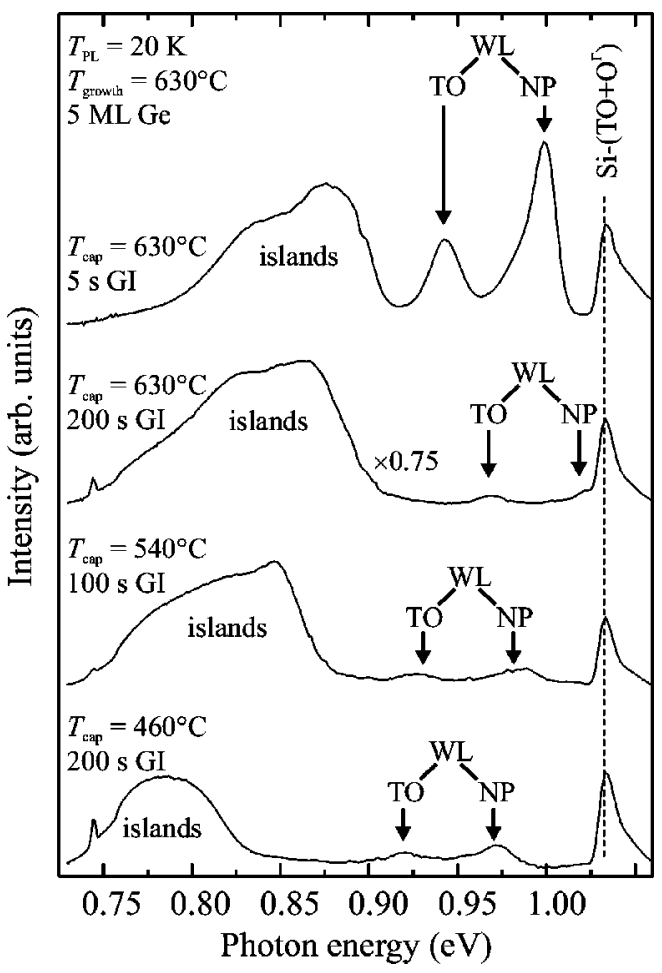

FIG. 50. Photoluminescence from dome-shaped SiGe islands as a function of growth temperature during Si capping. From Schmidt, Denker, et al., 2000.

crete zero-dimensional $s$ and $p$ levels of about $40-\mathrm{meV}$ energy separation.

The larger dome-shaped Ge islands with diameters around $100 \mathrm{~nm}$ and heights of about $10 \mathrm{~nm}$ have an effective valence-band offset of $300-400 \mathrm{meV}$, due to $\mathrm{Si}-\mathrm{Ge}$ intermixing and their strain status. Because of their larger size, lateral confinement effects are not observed, and dome-shape islands can be regarded as quantum wells with confinement only along the growth direction. The tensile in-plane strain in Si above and below the Ge domes leads to a lowering of the $\Delta(2)$ conduction band valleys. Figure 50 displays the photoluminescence from dome-shaped islands overgrown at 630, 540 , and $460^{\circ} \mathrm{C}$. The suppression of intermixing for lower capping temperatures leads to a pronounced redshift of the island photoluminescence. Together with information on the Ge profile as obtained from crosssectional TEM and XRD, the heavy-hole HH1 energy level and wave function can be calculated.

Room-temperature electroluminescence of both pyramid and dome-shaped islands embedded in the intrinsic region of a $p-i-n$ diode has been observed by Brunhes $e t$ al. (2000) and Vescan et al. (2003). The strongest emission was observed if five to ten island layers were stacked. Although Stoffel et al. (2003) succeeded in observing room-temperature electroluminescence from MBE-grown Ge hut clusters at photon energies of about $0.8 \mathrm{eV}$, it seems that room-temperature luminescence is easier to achieve with chemical-vapor-deposition-based methods than with molecular-beam epitaxy. 


\section{Investigations of single quantum dots}

Recently, investigations of single InGaAs quantum dots have attracted considerable attention (Gerard and Gayral, 1999; Chen et al., 2000; Michler et al., 2000; Guest et al., 2001; Moreau et al., 2001a, 2001b; Regelman et al., 2001; Santori et al., 2002). These studies eliminate inhomogeneous line broadening, which somewhat obscures the physics. With single-dot spectroscopy, one can study exchange and correlation phenomena, as well as electron or hole charging. Finley et al. (2003) employed Stark-effect spectroscopy of individual InGaAs dots, incorporated into a $p$-type Schottky photodiode structure. This provided a probe for neutral and positively charged excitons. In particular, the experiments revealed a reduction of the permanent excitonic dipole moment as additional holes were added to an island with a width of $22 \mathrm{~nm}$ and a height of $5 \mathrm{~nm}$. The reduction of the dipole moment was accompanied by a lateral expansion of the hole wave function and a corresponding contraction of the electron wave function. Whereas the experimental and theoretical values for the change of the dipole moment are in good agreement, it turns out that the width of the islands is much more important than the lateral profile.

Single dots and coupled dots offer the potential to realize future quantum information concepts based on coherent phenomena. Gerard and Gayral (1999) have pointed out that, with islands in a vertical cavity, $50 \%$ coupling efficiency is possible. Using pulsed laser excitation of single quantum dots they demonstrated a singlephoton turnstile device. A single InGaAs quantum dot was embedded in a high-quality microcavity structure and single photons were generated at the fundamental quantum-dot exciton transition.

The InGaAs quantum dot offers a two-level system, the coupled electron-hole pair of an exciton, which represents the two levels $|0\rangle$ and $|1\rangle$ of a qbit. Under optical excitation using picosecond laser pulses, such a system represents a driven single anharmonic quantum system, which exhibits a dead time between successive photon emission events (photon antibunching). Santori et al. (2002) showed that consecutive photons from such a source are largely indistinguishable, i.e., have nearly identical wave packets. By locating the GaInAs islands in the intrinsic region of a GaAs $p-i-n$ diode structure and biasing this structure, an electrically driven singlephoton source was realized by Yuan et al. (2002). The sequential emission of individual photons was proven by correlation spectroscopy. Going a step further, Zrenner et al. (2002) used coherent manipulation between the two excitonic energy levels (Rabi oscillations) in a single quantum dot under excitation with electromagnetic fields to convert the coherent optical excitation into a deterministic photocurrent, i.e., into an optically triggered single-electron turnstile (see also Bayer, 2002), which provides for an electric readout of excitonic quantum gates.

\section{CALCULATION OF THE STRAIN DISTRIBUTION WITHIN AND AROUND NANOSTRUCTURES}

An important driving force in self-organized growth is the local strain at the surface. The strain distribution in buried and uncapped islands substantially affects their electronic properties. The knowledge of the elastic strain field is also important for the analysis of x-ray and electron scattering data and for understanding the mechanisms of dot position correlation and creation of an equilibrium dot shape.

Two general approaches are used for the calculation of the elastic strain connected with self-assembled islands, namely, continuum elasticity and atomistic simulations. In the former method, the actual structure is replaced by an elastic continuum and the strain distribution is obtained by solving the elasticity equilibrium equation or by minimizing the elastic energy stored in the sample. For a nanostructure buried below a plain surface, or in the approximation of small slopes at the surface, analytical solutions of this problem can be found. In all other cases, a numerical finite-element method has to be used.

In the atomistic simulation methods, two- and manyatom potentials are used for the calculation of the elastic energy of a sample; the strain is obtained by minimizing this energy. In this approach, either empirical or semiempirical interatomic potentials are used or the potentials are calculated $a b$ initio using many-body quantummechanical methods.

In the following sections, we present the basic ideas and expressions of the calculation methods and discuss the validity of their results.

\section{A. Analytical methods in continuum elasticity}

The analytic method is based on the solution of the equilibrium equation

$$
\frac{\partial \sigma_{j k}}{\partial r_{k}}+f_{j}=0, \quad \mathbf{r}=\left\{r_{j}\right\}=(x, y, z), \quad j, k=x, y, z,
$$

where $\sigma_{j k}(\mathbf{r})$ are the components of the stress tensor in and around the island and $f_{j}(\mathbf{r})$ is the density of the volume force acting in point $\mathbf{r}$ in the sample. The stress tensor is connected by the elastic strain tensor $\hat{\varepsilon}$ by means of Hooke's law of linear elasticity,

$$
\sigma_{j k}=c_{j k l m} \varepsilon_{l m} .
$$

Here $c_{j k l m}$ are the four-index elastic coefficients $(j, k, l, m=x, y, z)$, which are usually written in the $6 \times 6$ notation as $C_{j k}(j, k=1, \ldots, 6)$. In this section, we choose the $x, y$, and $z$ axes parallel to the crystallographic $\langle 100\rangle$ directions. For a general orientation of the coordinate axes, an appropriate transformation of the elastic constants must be performed. In a cubic system and using the special orientation of the axes, only three coefficients, $C_{11}, C_{12}$, and $C_{44}$, are different from zero (see, for instance, Timoshenko and Goodier, 1971). The strain tensor components can be derived from the displace- 
ment vector $\mathbf{u}(\mathbf{r})$ defined with respect to the atomic sites of the nondeformed host material:

$$
\varepsilon_{j k}=\frac{1}{2}\left(\frac{\partial u_{j}}{\partial r_{k}}+\frac{\partial u_{k}}{\partial r_{j}}\right), \quad j, k=x, y, z \text {. }
$$

At first, we replace the actual island by a very small sphere placed in the origin $\mathbf{r}=0$ (a pointlike inclusion). Such a sphere acts as a force dipole, and the corresponding force density is

$$
f_{j}(\mathbf{r})=-p_{j k} \frac{\partial \delta(\mathbf{r})}{\partial r_{k}} .
$$

The dipole-moment tensor $p_{j k}$ depends on the symmetry of the lattice of the sphere and the surrounding crystal matrix. For a cubic defect in a cubic crystal matrix, this tensor is diagonal and

$$
p_{j k}=\Delta\left(C_{11}+2 C_{12}\right) \delta_{j k},
$$

where the elastic coefficients $C_{j k}$ are assumed to be the same for the inclusion and the matrix. Expressions for $p_{j k}$ for lower symmetries can be found, for instance, in Krivoglaz (1991). The constant $\Delta$ is the relative mismatch between the nondeformed lattice constant of the inclusion $a_{\text {incl }}$ and the surrounding crystal $a$,

$$
\Delta=\frac{a_{\text {incl }}-a}{a} .
$$

If the inclusion is buried at a given depth below the flat surface at $z=0$ of a semi-infinite crystal, Eq. (18) is solved with the boundary condition

$$
\sigma_{j z \mid z=0}=0
$$

expressing the fact that the surface is free. For an infinite crystal, the components of the stress tensor $\sigma_{j k}$ must vanish in a very large distance from the inclusion.

In the literature, several methods can be found for the solution of Eq. (18) with the force of Eq. (21). Dederichs and Leibfried (1969), Flocken and Hardy (1970), Masumura and Sines (1970), and Dederichs and Pollmann (1972) presented solutions for a pointlike inclusion embedded in an infinite crystal matrix with cubic symmetry; its displacement field is denoted by $\mathbf{w}(\mathbf{r})$. The authors found an explicit expression for the Fourier transformation of $\mathbf{w}(\mathbf{r})$ :

$$
\begin{aligned}
w_{j}^{\mathrm{FT}}(\mathbf{q})= & -i \frac{\Delta}{q}\left(C_{11}+2 C_{12}\right) \frac{q_{j}^{0}}{C_{44}+A\left(q_{j}^{0}\right)^{2}} \cdot[1 \\
& \left.+\sum_{m=x, y, z} \frac{C_{44}+C_{12}}{C_{44}+A\left(q_{m}^{0}\right)^{2}}\left(q_{m}^{0}\right)^{2}\right]^{-1}
\end{aligned}
$$

where $A=C_{11}-C_{12}-2 C_{44}$ describes the elastic anisotropy and $\mathbf{q}^{0}=\mathbf{q} / q$. Andreev et al. (1999) suggested a general procedure for the calculation of $\mathbf{w}^{\mathrm{FT}}$ for any crystal symmetry. Faux and Pearson (2000) expressed the displacement field $\mathbf{w}(\mathbf{r})$ of a pointlike inclusion in real space using a power series with the factor $t$ $=A /\left(C_{11}+2 C_{44}\right)$ :

$$
\mathbf{w}(\mathbf{r})=\sum_{n=0}^{\infty} \mathbf{w}^{(n)}(\mathbf{r}) t^{n}
$$

Faux and Pearson explicitly derived $\mathbf{w}^{(n)}(\mathbf{r})$ for cubic symmetry and discussed the importance of higher terms for the displacement calculation.

For an elastically isotropic continuum $(A=0)$ we obtain the simple result

$$
\mathbf{w}^{\mathrm{FT}}(\mathbf{q})=-i \Delta \frac{\mathbf{q}}{q^{2}} \frac{1+\nu}{1-\nu},
$$

where $\nu=C_{12} /\left(C_{11}+C_{12}\right)$ is the Poisson ratio. In an anisotropic medium, the Poisson ratio depends on direction, and the corresponding formulas can be found in Wortman and Evans (1965). With Eq. (27), the displacement field $\mathbf{w}(\mathbf{r})$ of a pointlike inclusion in real space can be calculated by performing the inverse Fourier transformation analytically,

$$
\mathbf{w}(\mathbf{r})=\frac{\Delta}{4 \pi} \frac{1+\nu}{1-\nu} \frac{\mathbf{r}}{r^{3}} .
$$

For a general anisotropic case and for any lattice with point symmetry, the displacement field in point $\mathbf{r}$ due to a pointlike inclusion in point $\mathbf{r}^{\prime}$ is given by $\mathbf{w}\left(\mathbf{r}-\mathbf{r}^{\prime}\right)$, i.e., it depends only on the relative position of the observation point and the inclusion.

For the calculation of the displacement field of an island of finite size embedded in an infinite anisotropic medium, the key assumption is that the elastic constants of the medium and the island are the same. Then the displacement $\mathbf{u}(\mathbf{r})$ in a given point $\mathbf{r}$ of the medium (inside or outside the island) can be expressed as a superposition of the displacements $\mathbf{w}\left(\mathbf{r}-\mathbf{r}^{\prime}\right)$ of pointlike inclusions filling the volume of the island (Andreev et al., 1999; Faux and Pearson, 2000):

$$
\mathbf{u}(\mathbf{r})=\int d^{3} \mathbf{r}^{\prime} \Omega\left(\mathbf{r}^{\prime}\right) \mathbf{w}\left(\mathbf{r}-\mathbf{r}^{\prime}\right),
$$

where the integral is calculated over the infinite $3 \mathrm{D}$ continuum and the function $\Omega\left(\mathbf{r}^{\prime}\right)$ is the shape function of an island (unity inside and zero outside). Since the resulting displacement field is a convolution of the displacement field $\mathbf{w}$ of a pointlike inclusion with the shape function of the island, the Fourier transformation of $\mathbf{u}(\mathbf{r})$ is given by the product of the Fourier-transformed quantities $\mathbf{w}^{\mathrm{FT}}(\mathbf{q})$ and $\Omega^{\mathrm{FT}}(\mathbf{q})$. This expression is used for the calculation of $\mathrm{x}$-ray scattering patterns (see Sec. III.E).

If the elastic constants of the island and the surrounding host lattice are different, the superposition assumption expressed by Eq. (29) is invalid. For this case, Andreev et al. (1999) suggested expressing the Fourier transformation of the resulting strain tensor by a perturbation series, with the perturbation parameter given by the difference of the elastic constants. However, neglecting the difference in elastic constants results in a negligible error in most cases (Keyes, 1962).

If the island is embedded in a semi-infinite medium with a flat surface at $z=0$, the translational symmetry 
yielding the convolution expression in Eq. (29) is violated in the $z$ direction perpendicular to the surface, and Eq. (29) has to be replaced by

$$
\mathbf{u}(\mathbf{r})=\int d^{3} \mathbf{r}^{\prime} \Omega\left(\mathbf{r}^{\prime}\right) \mathbf{w}\left(\mathbf{r}_{\|}-\mathbf{r}_{\|}^{\prime} ; z, z^{\prime}\right)
$$

where $\mathbf{r}_{\|} \equiv(x, y, 0)$ is the projection of the position vector $\mathbf{r}$ to the surface. The displacement field of a pointlike inclusion can be expressed by a closed analytical expression for an isotropic semi-infinite continuum only $(\mathrm{Hu}$, 1989):

$$
\begin{aligned}
\mathbf{w}\left(\mathbf{r}_{\|}-\mathbf{r}_{\|}^{\prime} ; z, z^{\prime}\right)= & \frac{\Delta}{4 \pi} \frac{1+\nu}{1-\nu} \cdot\left[\frac{\mathbf{R}_{1}}{R_{1}^{3}}+(3-4 \nu) \frac{\mathbf{R}_{2}}{R_{2}^{3}}-6 z\left(z+z^{\prime}\right)\right. \\
& \left.\times \frac{\mathbf{R}_{2}}{R_{2}^{5}}-\frac{2 \mathbf{n}}{R_{2}^{3}}\left[(3-4 \nu)\left(z+z^{\prime}\right)-z\right]\right] .
\end{aligned}
$$

Here $\mathbf{n}$ is the outward surface-normal vector and $\mathbf{R}_{1}=\mathbf{r}$ $-\mathbf{r}^{\prime}=\left(x-x^{\prime}, y-y^{\prime}, z-z^{\prime}\right), \mathbf{R}_{2}=\left(x-x^{\prime}, y-y^{\prime}, z+z^{\prime}\right)$ are the position vectors of the observation point $\mathbf{r}$ with respect to the point $\mathbf{r}^{\prime}$ in the island and to the corresponding point in a virtual island reflected at the free sample surface.

The first term in the square brackets in Eq. (31) expresses the displacement field of a pointlike inclusion in an infinite elastic continuum [compare Eq. (28)]; the other terms stem from the boundary condition Eq. (24), and they account for the elastic relaxation of internal stresses at the free surface.

Several authors have used Eqs. (30) and (31) to calculate the displacement field of islands with various shapes. Glas (2001) calculated the displacement field around a truncated fourfold pyramid and used this method for the evaluation of the elastic energy density at a free surface above a buried island (Glas, 2002). An expression similar to Eq. (31) was used by Pan (2002a, $2002 b$ ) for the calculation of the surface strain of islands buried in GaAs and GaN. In these papers, both the elastic and the piezoelectric surface fields were obtained by solving coupled elastic and piezoelectric equilibrium equations. Romanov et al. (2001) presented explicit expressions for the strain field around a buried island of ellipsoidal shape. Barabash and Krivoglaz (1987) used Eq. (31) for the calculation of the Fourier transformation of a displacement field caused by point inclusions randomly distributed in a subsurface layer with a given density depth profile. They found that the relaxation part of the displacement field was determined by the one-dimensional Laplace transformation of the inclusion density. Their calculations show that the surface relaxation affects the Fourier transformation $\mathbf{u}^{\mathrm{FT}}(\mathbf{q})$ of the total displacement field only for small space frequencies $|\mathbf{q}|_{\|}<2 \pi / D$, where $D$ is the thickness of the layer with inclusions.

The solution of the strain field of an island embedded in a semi-infinite anisotropic medium is possible in the approximation of equal elastic constants of island and medium, similar to the case of an infinite matrix. For the solution of Eq. (18) with the boundary condition in Eq.
(24), we assume cubic symmetry of the inclusion and the matrix. For this case, the dipole-moment tensor $p_{j k}$ is scalar and the volume force density is expressed as

$$
f_{j}(\mathbf{r})=-\left(C_{11}+2 C_{12}\right) \frac{\partial \Delta(\mathbf{r})}{\partial r_{j}},
$$

where the lattice mismatch $\Delta$ varies with the local chemical composition.

For the solution, we use an ansatz as a 2D Fourier integral,

$$
\mathbf{u}^{\mathrm{FT} 2}\left(\mathbf{q}_{\|} ; z\right)=\int d^{2} \mathbf{r}_{\|} \mathbf{u}(\mathbf{r}) e^{-\mathrm{i} \mathbf{q}_{\|} \cdot \mathbf{r}_{\|}} .
$$

Then the equilibrium equation is transformed into an ordinary differential equation of second order,

$$
\hat{\mathbf{A}}\left(\mathbf{u}^{\mathrm{FT} 2}\right)^{\prime \prime}+\mathbf{i} \hat{\mathbf{B}}\left(\mathbf{u}^{\mathrm{FT} 2}\right)^{\prime}-\hat{\mathbf{C}} \mathbf{u}^{\mathrm{FT} 2}=\mathbf{D} \text {. }
$$

The $3 \times 3$ matrices $\hat{\mathbf{A}}, \hat{\mathbf{B}}$, and $\hat{\mathbf{C}}$ contain the elastic constants $C_{11}, C_{12}$, and $C_{44}$, and the components $q_{x, y}$ of the reciprocal-space vector $\mathbf{q}_{\|}$, while the $3 \times 1$ vector $\mathbf{D}$ is the local lattice mismatch, Fourier transformed by Eq. (33). The primes denote the derivation with respect to $z$. The boundary conditions at the free and the rear sample surfaces $(z=0, z \rightarrow-\infty)$ can be written in matrix form as well:

$$
\hat{\mathbf{A}}\left(\mathbf{u}^{\mathrm{FT} 2}\right)^{\prime}+\left.i \hat{\mathbf{F}} \mathbf{u}^{\mathrm{FT} 2}\right|_{z=0, z \rightarrow-\infty}=0,
$$

where $\hat{\mathbf{F}}$ is a $3 \times 3$ matrix containing the $C_{j k}$ and $\mathbf{q}_{\|}$. Equation (34) along with the boundary conditions Eq. (35) can be solved by standard analytic methods. Details of the calculation can be found in Roch et al. (2002).

The method described above suffers from the restriction that the elastic constants of the island do not depend on its local chemical composition, but are (set) equal to those of the surrounding crystal. It has been proven numerically for several geometries and materials by Roch et al. (2002) that a spatial variation of the elastic constants can be included, approximately replacing the elastic constants in Eq. (32) by their local compositiondependent values:

$$
f_{j}(\mathbf{r})=-\frac{\partial}{\partial r_{j}}\left\{\left[C_{11}(\mathbf{r})+2 C_{12}(\mathbf{r})\right] \Delta(\mathbf{r})\right\},
$$

but keeping the elastic constant of the host material in the expression for the stress tensor $\sigma_{j k}$ in the first term of Eq. (18).

As an example, Fig. 51 shows the distribution of $\varepsilon_{x x}$ and $\varepsilon_{z z}$ in and around a Ge island buried below the $\mathrm{Si}(100)$ surface. The island has the form of a pyramid with a square base and $\{105\}$ facets as sidewalls. We assume a Ge content of $100 \%$ throughout the pyramid; the strain tensor is defined with respect to the nondeformed Si lattice.

From Fig. 51, it follows that the surface relaxation of internal stresses affects the strain distribution mainly above the apex of the pyramid. The lateral strain $\left(\varepsilon_{x x}\right)$ exhibits a local maximum at the surface above the is- 


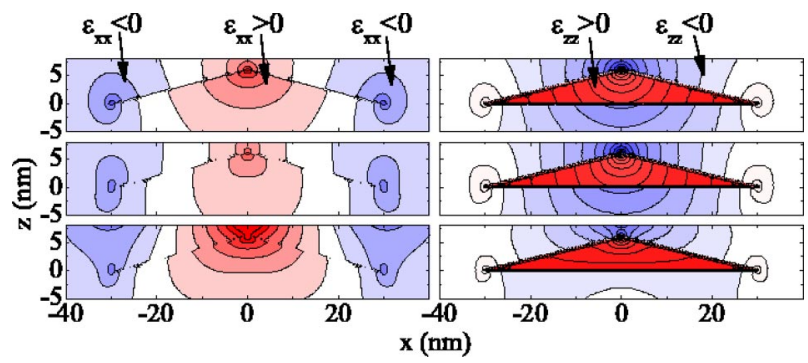

FIG. 51. (Color in online edition) Strain tensor components $\varepsilon_{x x}$ (left) and $\varepsilon_{x x}$ (right) in and around a Ge pyramid buried below a Si surface. The strain distributions in the upper row have been calculated assuming an infinite isotopic Si matrix [Eqs. (27) and (29)]. In the middle row an anisotropic infinite continuum was assumed [Eqs. (25) and (29)]. In the lower row the strain calculation in a semi-infinite anisotropic continuum was performed using Eqs. (32) and (35). The step of the contours is $\Delta \varepsilon=0.002$. The upper edges of the graphs correspond to the free surface.

land, which is caused by the stress relaxation as well. The maximum lateral strain in the $\mathrm{Ge}$ island is about $1.5 \%$, while the vertical strain in the pyramid nearly corresponds to the Ge-Si mismatch $(\approx 4 \%)$. The anisotropy of elastic constants has a minor effect in the case of $\mathrm{Si}$; for other materials like III-V compounds, however, the elastic anisotropy is more important. Glas (1991) and Faux (1994) solved the same problem using Airy stress functions and assuming elastic isotropy of the host lattice and obtained similar results.

Figure 52 presents a distribution of the strain components $\varepsilon_{x x, z z}$ calculated along the vertical axis of a stack of $10 \mathrm{Ge}$ islands in a semi-infinite Si matrix. For the calculation, the above continuum approach was applied, taking into account both the elastic anisotropy and the surface stress relaxation. The maximum of $\varepsilon_{z z}$ reaches about $6 \%$, which corresponds to the $\mathrm{Ge}-\mathrm{Si}$ mismatch corrected by the tetragonal-distortion term $(1+\nu) /(1$ $-\nu)$. Between the islands, the Si lattice is vertically compressed; this compression is reduced close to the free surface due to the surface relaxation. The surrounding lattice vertically compresses the island lattice, which is obvious from the $z$ dependence of the maximum values

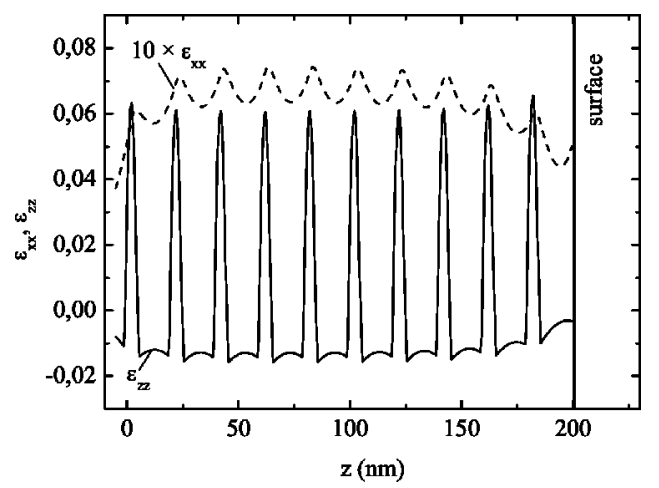

FIG. 52. Strain tensor components $\varepsilon_{x x}$ and $\varepsilon_{z z}$ calculated along the growth axis of a tenfold stack of Ge islands in a multilayer. of $\varepsilon_{z z}$ in the islands. The lateral strain component is positive both in the islands and between them, and its value is reduced at the surface due to the surface relaxation (Darhuber et al., 1997; Holý et al., 1998c; Schmidt and Eberl, 2000).

The analytic methods for the calculation of the strains in and around an island are limited to the case of an ideally flat surface. For the calculation of uncapped islands, a numerical method for solving Eq. (18) has to be applied instead. However, for a rough estimate of the elastic energy stored in an island at a free surface, the equilibrium equation Eq. (18) can be solved analytically in the small-slope approximation (see, for instance, Budiman and Ruda, 2000; Spencer et al., 2001). Kaganer and Ploog (2001) used another approach for the calculation of the elastic strain in an uncapped island, describing it as a stack of circular monolayers and evaluating the contributions of individual monolayer steps to the total strain field. A semianalytic calculation of the displacement field in an uncapped island with homogeneous chemical composition has been presented by Spencer and Meiron (1994) without assuming a small sidewall slope. The calculation is based on a numerical solution of an integral equation containing two analytic complex functions (the Goursat functions); Spencer and Tersoff (2001) used this method to calculate the displacement field for a 2D model.

\section{B. Numerical methods in continuum elasticity}

The strain status of an uncapped or buried island can be calculated numerically by minimizing the elastic energy stored in the system. The elastic energy density is given by

$$
\mathcal{E}_{\mathrm{el}}(\mathbf{r})=\frac{1}{2} c_{j k l m}(\mathbf{r}) \varepsilon_{j k}(\mathbf{r}) \varepsilon_{m n}(\mathbf{r})
$$

To include the lattice mismatch between the island and its surroundings, we define the strain tensor with respect to the lattice sites of the nondeformed host material. The island lattice is expanded/contracted with respect to the host lattice due to the lattice mismatch, which lowers the elastic energy stored in the island by the factor

$$
-p_{j k}(\mathbf{r}) \varepsilon_{j k}(\mathbf{r}) .
$$

For cubic symmetry, this factor is

$$
-\left[C_{11}(\mathbf{r})+2 C_{12}(\mathbf{r})\right] \Delta(\mathbf{r}) \operatorname{Tr}(\hat{\varepsilon}),
$$

where $\operatorname{Tr}$ denotes the trace of the tensor, and we also account for the influence of the local chemical composition on the elastic constants (Pryor et al., 1998).

The minimization of the elastic energy is performed by a discretization of the displacement field $\mathbf{u}(\mathbf{r})$ and a replacment of the derivatives in Eq. (20) by a suitable difference scheme (the finite-element method). The boundary conditions are introduced either by putting $\mathbf{u}$ $=0$ in the substrate far away from the island or using the fact that no external force is acting on the free surface, 


$$
\sigma_{j k} n_{k \mid \text { surface }}=0,
$$

where $\mathbf{n}$ is the unit vector of the surface normal.

The finite-element method is often used for selfassembled semiconductor nanostructures. Carlsson et al. (1998) calculated the strain fields in an uncapped InP quantum dot on a GaInP substrate. The authors compared the calculated strain field to data from highresolution TEM cross sections of the dot and achieved a very good correspondence. This demonstrates that the bulk values of the elastic constants $C_{j k}$ used in finiteelement-method calculations are also valid for the description of the elastic properties of nanoscale islands.

The elastic energy density at a free flat surface above a buried island was calculated by this method as well. As we have shown in Sec. II.C, the strain distribution affects the probability of island nucleation and consequently determines the correlation of island positions at different interfaces. Benabbas et al. (1999) used the strains in and around buried InAs islands in GaAs for the simulation of growth and vertical island pairing probability in an InAs/GaAs multilayer, which was found to depend heavily on the thickness of the GaAs spacer layers. A similar study was performed by Jogai (2002) for InGaAs islands in a GaAs matrix. The dependence of the surface strain energy distribution on the shape of buried Ge islands in $\mathrm{Si}$ was studied by Priester (2001). Due to elastic anisotropy, additional minima in the surface distribution of the elastic energy density occur for pairs of InAs islands embedded in GaAs (Jogai, 2000). Moll et al. (1998) combined the results from finiteelement-method calculations of elastic strains in uncapped InAs islands with atomistic simulations of the surface energy of the island facets and found an equilibrium island shape. Jogai (2001) used the finite-element method for the calculation of strains in and around a hexagonal InN island embedded in AlN. From the strain data, the piezoelectric fields were calculated, but no coupling between the piezoelectric field and the strain field was considered.

Additionally, finite-element-method simulations of strains have served as the basis for calculation of the electronic band structure [see, for instance, Schmidt, Eberl, and Rau (2000) for Ge dots in Si and Holm et al. (2002) for InAs islands in InP]. Finite-element results have also been used for the simulation of x-ray diffraction from uncapped and buried islands (Wiebach et al., 2000; Stangl, Daniel, et al., 2001; Hesse et al., 2002; Schmidbauer, Hatami, et al., 2002), and for the interpretation of TEM images (see Carlsson et al., 1998; Kret et al., 1999; Rosenauer et al., 2000).

\section{Atomistic simulations}

Similarly to the previous method, the atomistic approach is based on the minimization of the total elastic energy, but the continuum elasticity expression (36) is replaced by the superposition of the energy contributions of atomic pairs, triplets, etc.:

$$
\begin{aligned}
E_{\mathrm{el}}= & \sum_{j \neq k} \phi^{(2)}\left(\mathbf{R}_{j}-\mathbf{R}_{k}\right)+\sum_{j \neq k \neq m} \phi^{(3)}\left(\mathbf{R}_{j}-\mathbf{R}_{k}, \mathbf{R}_{j}-\mathbf{R}_{m}\right) \\
& +\cdots
\end{aligned}
$$

where $\phi^{(n)}$ denotes the $n$-atom contribution to the total energy, $\mathbf{R}_{j}$ is the position of the $j$ th atom, and the sums run over pairs of atoms, triplets, etc. The minimum of the elastic energy is found numerically; typically millions of atoms have to be considered. Either the atomic potentials are calculated $a b$ initio or the form of the potentials $\phi^{(n)}$ is chosen so that the atomistic simulations yield the same elastic properties as in conventional continuum elasticity. Even in this case, there are several important differences between the atomistic and continuum methods (Pryor et al., 1998). The atomistic approach accounts for the true point symmetry of the elementary unit cell of the material, while the elastic constants depend only on the crystal system. This can be important, for instance, in the case of GaAs with a point group $\overline{4} 3 m$ that does not contain the fourfold rotational axis. Its elastic constants $C_{11}, C_{12}$, and $C_{44}$, however, have the full fourfold symmetry characteristic of the cubic system. Another important difference lies in the harmonicity assumption. The usual formulation of continuum elasticity is based on Hooke's law linearly connecting strains with stresses. A properly chosen atomistic model includes the nonlinear behavior of the lattice.

In the literature, several types of potentials $\phi^{(n)}$ can be found. In the pioneering works of Musgrave and Pople (1962), Keating (1966), and Martin (1970), the valenceforce-field model was developed for crystals with diamond structure. In this model, Eq. (40) is simplified, taking into account the interaction only of nearest neighbors, but neglecting terms with $n>3$ as well as the long-range Coulomb interaction between charged ions. In the notation used by Pryor et al. (1998), the total elastic energy is given by

$$
\begin{aligned}
E_{\mathrm{el}}= & \frac{1}{2} \sum_{j}\left[\sum_{k}^{\mathrm{nn}} \frac{3 \alpha_{j k}}{8 d_{j k}^{2}}\left[\left|\mathbf{R}_{j}-\mathbf{R}_{k}\right|^{2}-d_{j k}^{2}\right]^{2}\right. \\
& +\sum_{k, m>k}^{\mathrm{nn}} \frac{3 \beta_{j, k m}}{8 d_{j k} d_{j m}}\left[\left(\mathbf{R}_{k}-\mathbf{R}_{j}\right) \cdot\left(\mathbf{R}_{m}-\mathbf{R}_{j}\right)\right. \\
& \left.-d_{j k} d_{j m} \cos \left(\theta_{j, k m}\right)\right]
\end{aligned}
$$

Here $d_{j k}$ denotes the nondeformed bond length between atoms $j$ and $k$, and $\theta_{j, k m}$ is the nondeformed bond angle of the triplet of atoms $k, j, m$. The sums over $k$ and $m$ include the nearest neighbors of atom $j$. For diamond or zinc-blende structure, the ideal bond length is $d=a \sqrt{3} / 4$ ( $a$ is the lattice parameter), and the ideal bond angle is given by $\cos \theta=-1 / 3$. Because of symmetry, the constants $\alpha_{j k}$ and $\beta_{j, k m}$ do not depend on the indexes $j, k$, and $m$ and their values are determined from a fit of the total energy to its continuum description for a bulk material. From this fit, the following expressions were obtained (Keating, 1966; Pryor et al., 1998): 


$$
\begin{aligned}
& C_{11}=\frac{\alpha+3 \beta}{a}, \\
& C_{12}=\frac{\alpha-\beta}{a}, \\
& C_{44}=\frac{4 \alpha \beta}{a(\alpha+\beta)} .
\end{aligned}
$$

Martin (1970) took into account the Coulomb interaction between the ions in a zinc-blende structure and obtained

$$
\begin{aligned}
& C_{11}=\frac{\alpha+3 \beta}{a}-0.083 \xi, \\
& C_{12}=\frac{\alpha-\beta}{a}-0.136 \xi, \\
& C_{44}=\frac{\alpha+\beta}{a}-0.136 \xi-\frac{[(\alpha-\beta) / a-0.294 \xi]^{2}}{(\alpha+\beta) / a-0.266 \xi},
\end{aligned}
$$

where $\xi=(Z e)^{2} /\left(\epsilon d^{4}\right), \pm Z e$ is the ion charge in cgs units and $\epsilon$ is the relative permittivity.

In Eqs. (42) and (43) three elastic constants $C_{11,12,44}$ depend on only two free parameters, $\alpha$ and $\beta$. Nevertheless, for a zinc-blende material, $\alpha$ and $\beta$ can be chosen so that the corresponding $C_{j k}$ values fit to the measured values with an error of a few percent. Martin (1970) presented the values of $\alpha$ and $\beta$ together with the charge parameter $Z^{2} / \epsilon$ for several semiconductor materials.

Beside the valence-force field model, other empirical potentials can be found in the literature. The StillingerWeber potential (Stillinger and Weber, 1985) is often used for atomistic simulations in $\mathrm{Si}$ or Ge. This potential is chosen so that it correctly describes not only the elastic properties of a solid crystal but also its melting. Kikuchi et al. (2001) compared the valence-force field and Stillinger-Weber methods by calculating the strain field in and around a pyramidal SiGe island at the Si surface or buried in a semi-infinite Si substrate. Both methods yielded very similar results except for the substrate and at the edges of an uncapped island. The strain profile in the substrate obtained by the valence-force field method exhibited nonrealistic oscillations; therefore the Stillinger-Weber potential is recommended for small islands with widths below $10 \mathrm{~nm}$. Baskes (1987) compared several empirical interatomic potentials for Si.

For $\mathrm{Si}, \mathrm{Si}-\mathrm{Ge}$, and $\mathrm{Si}-\mathrm{C}$, the interatomic potentials proposed by Tersoff $(1988,1989)$, which describe very well both solid and molten materials, are widely used nowadays.

For the GaAs/InAs system, interatomic potentials have been suggested by Ebbsjoe et al. (2000) and $\mathrm{Su}$ et al. (2003). These potentials include steric repulsion, Coulomb interaction due to the bond ionicity, and the van der Waals interaction. In Su et al. (2003) these potentials were used for the calculation of the surface energy density, since they also describe correctly dimers at the free

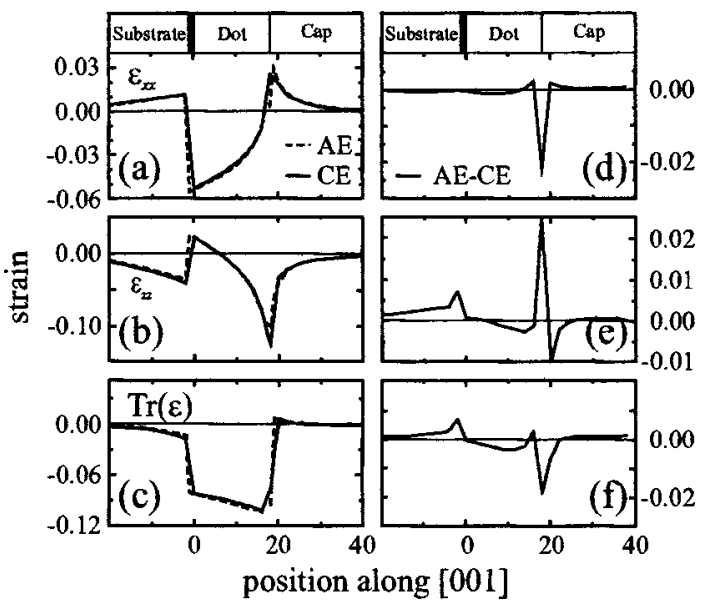

FIG. 53. Strain tensor components along the axis of a square InAs pyramid with $\{110\}$ side facets buried in GaAs: solid lines, obtained by valence force field approach; dashed lines, obtained by finite-element method. Panels (a)-(c) show the strain distributions, panels (d)-(f) the differences of both curves. The strains are defined with respect to unstrained InAs. From Pryor et al., 1998.

surface. From the calculations the length of the dimers was determined and it corresponds well to the results of $a b$ initio calculations. These potentials were also used for the simulation of the deformation field in an uncapped InAs island. The authors demonstrated that an island larger than approximately $40 \mathrm{~nm}$ contains several stress domains, whereas smaller islands have only a single stress domain.

The question of the validity of continuum elasticity for very small islands is often raised. Pryor et al. (1998) calculated the strain distribution in and around a pyramidal InAs dot buried in GaAs both by continuum calculations and atomistically using the valence-force field method. The constants $\alpha$ and $\beta$ were chosen so that they corresponded to the used values of $C_{11,12,44}$. Pryor et al. noted a good correspondence of both methods; discrepancies were found, however, in the regions with large strain. In Fig. 53, the strain tensor components along the pyramid axes obtained by finite-element and valenceforce field methods are plotted. The relative difference in the results can reach about $4 \%$ in the region where nearly full elastic relaxation is observed $(\varepsilon \approx 0.07)$, which is attributed to the deviation from linearity assumed in continuum elasticity. Comparing the Stillinger-Weber empirical potential to analytical calculations using Eqs. (30) and (31), Daruka, Barabasi, et al. (1999) found good agreement between the two calculations of the surface strain distributions due to buried Ge islands in $\mathrm{Si}$. A similar finding was made by Makeev and Madhukar (2003), who calculated the strain field around a spherical Ge inclusion in a semi-infinite $\mathrm{Si}$ substrate numerically using the Stillinger-Weber potential functions and the continuum approach.

Instead of using empirical potentials, one can apply first-principles quantum-mechanical calculations to describe the properties of islands. $A b$ initio calculations 
based on the local-density approximation were used for the calculation of the equilibrium structure of silicon (Yin and Cohen, 1982; Nelds and Martin, 1984) as well as for nanostructures (Buda et al., 1992; Saitta et al., 1996). Baskes (1987) compared the elastic energy of $\mathrm{Si}$ calculated by several empirical atomic models with $a b$ initio studies. So far, the latter can be performed for a limited number of atoms and are very time consuming, even for very small islands with a size of about $10 \mathrm{~nm}$ $\left(\sim 10^{5}\right.$ atoms).

To reduce computing times, Lidorikis et al. (2001) proposed a hybrid atomistic calculation scheme in which the positions of the atoms close to a buried island are calculated using an atomistic approach and the displacement of more distant parts of the lattice are obtained using the finite-element method. Special attention must be paid to the "handshake region" connecting the atomistic and continuum calculation areas. The applicability of this method was demonstrated for the calculation of strains around a $\mathrm{Si}_{3} \mathrm{~N}_{4}$ inclusion in $\mathrm{Si}$ (Lidorikis et al., 2001).

Atomistic calculations have been used for various material systems. Groenen et al. (1999) applied the valenceforce field method for the evaluation of strains in and around InAs islands in InP. Makeev and Madhukar (2001) and Makeev et al. (2003) used the atomistic model with the Stillinger-Weber potential for the calculation of the hydrostatic stress caused by a Ge island buried in Si. The value of hydrostatic stress at the surface above the island apex varies as $D^{-1}$, where $D$ is the thickness of the spacer layer. This behavior cannot be explained by a continuum elasticity model with a pointlike inclusion, which rather predicts a $D^{-3}$-dependence. However, if one takes the true island size into account, a dependence close to $D^{-1}$ is obtained from Eqs. (32)-(35). In Makeev et al. (2003) the dependence of the in-plane strain at a free Si surface on the depth $D$ of a Ge pyramid buried underneath was compared with experimental data obtained by Schmidt and Eberl (2000) using the energy shift of the photoluminescence lines. The theoretical $D^{-1}$ dependence was fully confirmed by photoluminescence measurements.

Saito and Arakawa (2002) used the Keating valenceforce field method for the calculation of strains in hexagonal, prismatic, and pyramidal-shaped InGaN island in a hexagonal $\mathrm{GaN}$ layer. Their calculations took piezoelectric fields into account, using the finite element method and a tight-binding scheme for band-structure simulations. From molecular-dynamics simulations using a Stillinger-Weber potential, Su et al. (2001) predict two stress domains in large ( $\geqslant 40 \mathrm{~nm})$ uncapped InAs islands. Migliorato et al. (2002) used Tersoff's potential for the calculation of strains in InGaAs islands buried in a GaAs matrix before and after cleavage. They showed that the cleavage plane is elastically relaxed, creating a "buckle" similar to that observed by cross-sectional STM (Liu, Tersoff, et al., 2000). Their results support the concept of a high-concentration In core in a buried InGaAs island (Liu, Tersoff, et al., 2000). Valence-force field calculations of the strain in buried SiGe islands by Seok and Kim (2001) were used for electronic bandstructure calculations, finding strong evidence for a SiGe intermixing. Similar findings were published by Chaparro et al. (1999), indicating that the intermixing is driven by local strains at the island perimeter.

\section{IMPORTANT MATERIALS SYSTEMS}

In this section, we outline the properties and main applications of various materials systems. This survey cannot be complete. It is intended only as a starting point for further reading. In the first two sections, we summarize the results obtained for $\mathrm{SiGe}$ on $\mathrm{Si}$ and InAs on GaAs, our model systems in the sections above. Then, we outline the key properties of several other important semiconductor systems.

\section{A. SiGe on Si}

The driving force for island formation is relief of strain, and strain has a very important impact on the structural properties of islands. In $\mathrm{SiGe}$ on $\mathrm{Si}$, the main formation process for islands is Stranski-Krastanow growth. The interplay between reduction of strain energy and increase of surface energy accompanying island formation leads to different island shapes. If $\mathrm{SiGe}$ with low Ge composition is deposited, islands form without a nucleation barrier (Sutter and Lagally, 2000; Tromp et al., 2000; Tersoff et al., 2002). As shown in Sec. II.B, first, shallow mounds start to grow, and their sidewall angle gradually increases, until at about $11^{\circ}$ the total energy of the mounds is equal to that of $\{105\}$ facetted pyramids with the same sidewall angle. For larger islands, the latter shape has the lower energy, and consequently pyramids grow under further deposition of SiGe. At even higher volumes, it becomes energetically favorable to form steeper side facets, and a transition occurs from $\{105\}$ pyramids to domes with additional $\{113\}$ and $\{15323\}$ facets and, eventually, a (001) top facet.

For the deposition of $\mathrm{Ge}$ or SiGe with large Ge content on $\mathrm{Si}$, the situation is different: Now $\{105\}$ facetted islands are favored even for small island volumes. However, the increase in surface energy has to be overcome, and hence a nucleation process with a certain energy barrier dominates island formation (Jesson et al., 2000). For increasing amounts of Ge deposition, again a transition to domes occurs, but at lower island volumes compared to growth at low misfit. Under favorable growth conditions using gas-source $\mathrm{MBE}$ at $600^{\circ} \mathrm{C}$ substrate temperature, extremely size-uniform (height deviation of about $\pm 3 \%$ ) dome-shaped $\mathrm{Ge}$ islands were prepared by Jin et al. (2003).

During capping with $\mathrm{Si}$, the islands intermix, which reduces the strain. Consequently, a reverse shape transition occurs from domes to pyramids and even back to shallow mounds (Rastelli et al., 2001). Intermixing during capping can be suppressed by growth at low temperatures. 
Because intermixing reduces strain, this process already occurs during growth, raising the question of mass transport at the growing surface. Liao et al. (1999), Chaparro et al. (2000a), and Seta et al. (2002) have observed the formation of a trench around the island perimeter, indicating that $\mathrm{Si}$ from the island's surrounding is diffusing into the island. Consequently, even if pure Ge is deposited, as-grown islands exhibit a Ge composition of only about $50 \%$ at their base, and a trench is formed around their perimeter. At the apex, the Ge content of larger domes may reach 100\% (Stangl, Daniel, et al., 2001). Larger domes are elastically relaxed at their apex; therefore Ge adatoms prefer to "stick" there. During capping, Si actually dissolves the apex, and a flat (001) facet forms, while the material is incorporated at the island's side facets. Therefore during capping both an increase of the island base width and a decrease in the island height is observed (Sutter and Lagally, 1998).

Although strain plays a dominant role for structural island properties, other factors have to be considered, too. For instance, alloying SiGe with small amounts of carbon was thought to increase the flexibility in strain design, as carbon imposes strong tensile strain in SiGe. Although $\mathrm{C}$ is not miscible with $\mathrm{Si}$ like $\mathrm{Ge}$, with growth methods such as MBE, the deposition of $\mathrm{SiGeC}$ alloys is possible. Schmidt et al. (1997) tried to use C for SiGeC islands. A fractional monolayer of $\mathrm{C}$ was deposited onto $\mathrm{Si}$ before $\mathrm{Ge}$ to form islands. Indeed, islands with a base width of only about $10 \mathrm{~nm}$ and a height of about $1 \mathrm{~nm}$ were observed via TEM and AFM. A relatively strong photoluminescence signal from island multilayers was observed, and the energy shifts as a function of $\mathrm{Ge}$ deposition were interpreted as due to a spatially indirect recombination between holes confined in the Ge-rich island apex and electrons confined in the $\mathrm{SiGeC}$ island base. However, it turned out that the island formation was actually more complicated, and not only strain but also the bond chemistry between $\mathrm{Si}, \mathrm{Ge}$, and $\mathrm{C}$ needed to be considered. Leifeld et al. (2002) found that the predeposition of $\mathrm{C}$ leads to C-rich "patches" on the surface, with a $4 \times 4$ reconstruction. Ge adatoms do not stick to these patches, but, despite an unfavorable tensile strain, rather wet the $2 \times 1$ reconstructed $\mathrm{Si}$ surface between them, where the bond lengths and angles are more favorable (Kelires, 1998). Hence islands form in a Vollmer-Weber mode, i.e., without a $2 \mathrm{D}$ wetting layer, only in between the C-rich patches due to the chemical repulsion between $\mathrm{Ge}$ and $\mathrm{C}$. This explains the observed small island sizes as well as the photoluminescence properties. Recent Monte Carlo simulations (Hadjisavvas et al., 2003) of the stresses and compositions of such islands show that they are free of $\mathrm{C}$ atoms for any realistic growth conditions, and they exhibit an enhanced $\mathrm{Ge}$ content as compared to the $\mathrm{C}$-free case.

Furthermore, the nucleation of these islands is governed by the statistical distribution of C-rich patches rather than by the strain fields from buried islands. Hence no vertical position correlation is observed.

\section{B. InAs on GaAs}

InAs on GaAs is the model system for StranskiKrastanov growth and island formation. Due to the large lattice mismatch of $7 \%$ between InAs and GaAs, the strain in thin layers is larger than for most other semiconductor systems. Therefore, islanding sets in even below 2 ML's. The elastic relaxation is very pronounced, and islands adopt a rather high aspect ratio for small island volumes: typical island sizes are $30 \mathrm{~nm}$ base width and $11 \mathrm{~nm}$ height for MBE growth at $530^{\circ} \mathrm{C}$.

Cullis et al. (2002) found that a remarkably simple rule for the deposition of GaInAs can be formulated: if due to segregation an In concentration at the growing surface in excess of $85 \%$ is reached, islanding starts.

The pronounced elastic relaxation facilitates strain studies not only by TEM or XRD, but also by crosssectional STM, which relies on a detectable bulging of the cleavage face. Because Ga and As differ by only two positions in the periodic table, i.e., they exhibit nearly the same electron density, weak reflections like the (200) exist for the zinc-blende lattice. This fact helps to discriminate the scattered intensities stemming from InAs-rich regions from those of GaAs-rich regions, a feature used both in TEM and in XRD investigations. Consequently the distribution of the chemical composition within the islands has been established by crosssectional STM, by digital image analysis of TEM data, by energy-selective imaging in TEM, and by isostrain contours in x-ray grazing-incidence diffraction.

Many effects have been observed in the InAs/GaAs system for the first time, like vertical stacking of islands in multilayers, the facetting of uncapped islands, and their lateral ordering in the $\langle 100\rangle$ direction with the smallest values of Young's modulus in the (001) plane.

Despite the large number of investigations on the growth of InAs islands, a number of open problems still exist (Joyce, 2003):

(i) The Stranski-Krastanow growth mode of InAs on GaAs depends on the substrate orientation and its reconstruction. Stranski-Krastanow islands occur only on (001) and (111) $B$ surfaces, not on (110) and (111) $A$. However, InAs quantum dots can also be obtained on (110) GaAs surfaces if a thin AlAs layer is deposited before the island growth (Wasserman et al., 2003).

(ii) The 2D-3D transition is rather abrupt; consequently a large number of In $(\mathrm{Ga})$ atoms must be available for island nucleation (Krzyzewski et al., 2002a).

(iii) The chemical composition of the islands depends substantially on the deposition rate. The islands typically contain $\mathrm{Ga}$, but for slow deposition rates less $\mathrm{Ga}$ is incorporated, whereas for fast growth rates the amount of $\mathrm{Ga}$ in the islands is larger.

(iv) Significant $\mathrm{Ga}$ alloying is specific for the growth on a $\operatorname{GaAs}(001) c(4 \times 4)$-reconstructed surface. 
An AFM study of self-assembled InAs islands on GaAs by Ebiko et al. $(1998,1999)$ indicates that the island size distribution $N(s)$ follows a general scaling law [Eq. (6) in Sec. III.B]. Hence growth kinetics dominate the distribution of island sizes rather than strain. Krzyzewski et al. (2002b) found that this scaling law applies only in the saturation regime, when the number of islands has stabilized. Strain is important for the island size distribution in the very first growth stage.

Furthermore, since for InAs the minimum of the conduction band and the maximum of the valence band both occur at the center of the Brillouin zone, InAs islands are well suited for optical applications. The direct energy gap is $0.42 \mathrm{eV}$ (low $T$ ) to $0.35 \mathrm{eV}(300 \mathrm{~K})$; the corresponding values for GaAs are $1.52 \mathrm{eV}$ (low $T$ ) to $1.42 \mathrm{eV}(300 \mathrm{~K})$. Equally important is the type-I alignment of the $\mathrm{GaAs} / \mathrm{InAs} / \mathrm{GaAs}$ heterostructure, which leads to carrier confinement within the InAs islands for both holes and electrons. Capped InGaAs dots in GaAs are typically 2 to $5 \mathrm{~nm}$ high and have a base width of about $20 \mathrm{~nm}$. Their density is in the range of $10^{10}$ to $10^{11} \mathrm{~cm}^{-2}$.

With strain tuning, the realization of emitters or absorbers at long wavelengths up to $1.5 \mu \mathrm{m}$ is possible, but a high In content in the buried islands is a prerequisite (Liu et al., 2003). This high content is maintained by capping of the islands with an AlGaInAs alloy instead of pure GaAs.

As shown by Bester et al. (2003) and Bester and Zunger (2003), detailed atomistic pseudopotential manybody calculations of charged exciton recombination in buried InGaAs dots provide a link between the geometry and composition of the islands and their excitonic spectra.

\section{GaN}

Nitride-based dots are grown both in the hexagonal wurtzite (WU) phase or in their cubic zinc-blende (Z.B.) phase on appropriate substrates, which are typically $6 \mathrm{H} \mathrm{SiC}$ for the WU islands and either $3 \mathrm{H} \mathrm{SiC}$ or $\mathrm{GaAs}$ for the Z.B. islands. Several growth mechanisms are employed, like plasma-assisted MBE (Adelmann et al., 2000; Brault et al., 2003; Cho et al., 2003), OMCVD (Miyamura et al., 2002), or vapor-liquid-solid growth, in which $\mathrm{Ga}$ droplets are first deposited, then nitridated with $\mathrm{NH}_{3}$ (Cho et al., 2003; Hu et al., 2003). The GaN WU islands, embedded in AlN layers, have a luminescence energy in the range of about $3.5-4.2 \mathrm{eV}$ (Miyamura et al., 2002), which is decreased due to piezoelectric fields as compared to the bare energy gap. For calculation of the piezoelectric fields, coupled equations for piezoelectric and elastic fields have to be considered.

Piezoelectric fields are absent for cubic GaN in AlN layers (Martinez-Guerrero et al., 2000), for which photoluminescence at $3.8 \mathrm{eV}$ was reported. Typical $\mathrm{GaN}$ island sizes range from about 1.5 to $2 \mathrm{~nm}$ in height and from 5 to $20 \mathrm{~nm}$ in width for growth temperatures around $750^{\circ} \mathrm{C}$. Island densities up to $10^{11} \mathrm{~cm}^{-2}$ were reported. Stacked island layers have been grown as well, and Martinez-Guerrero et al. (2000) have observed oblique replication of GaN embedded in AlN along the cubic [001] direction for growth on $3 \mathrm{H} \mathrm{SiC}$ substrates. This oblique replication is not caused by strain fields of buried islands, but rather originates from bands of stacking faults within the $\{111\}$ planes. For WU GaN island multilayers, vertical correlation along the hexagonal [0001] growth direction was observed. Strain measurements performed with Raman spectroscopy (Gleize et al., 2001) and by grazing-incidence diffraction (Chamard et al., 2001) have shown that the mean in-plane strain within the $\mathrm{GaN}$ islands approaches that of the misfit between GaN and AlN. Despite the large density of threading dislocations, GaN/AlN island multilayers show a pronounced strain-induced vertical ordering of the GaN dots (Chamard et al., 2003), which is explained by the large elastic anisotropy in this hexagonal system. As far as stimulated emission is concerned, GaN islands embedded in AlN effectively confine carriers, which are not trapped by threading dislocations inside the islands (Cho et al., 2003). On the other hand, in wetting layers such defects serve as an effective drain for the carriers where they recombine nonradiatively. Much lower photoluminescence emission energies, in the range between 2.2 and $2.4 \mathrm{eV}$, are obtained if $\mathrm{GaInN}$ is deposited on top of cubic GaN layers (Husberg et al., 2001).

\section{InAs on $\ln P$}

InAs deposited on $\operatorname{InP}(001)$ substrates or $\operatorname{InP}(001)$ buffer layers gives rise to self-assembled islands as well as to self-assembled quantum wires along the [110] direction (González et al., 2000) due to the pronounced anisotropy of InP. The electronic structures of InAs islands on InP with anisotropies taken into account have been calculated in the $\mathbf{k} \cdot \mathbf{p}$ framework including piezoelectric fields by Holm et al. (2002).

\section{E. InP and GalnP}

The visible spectral range is accessible with InP quantum dots. Red-emitting islands can be grown either with OMCVD or with MBE at temperatures beyond $550{ }^{\circ} \mathrm{C}$ or around $500{ }^{\circ} \mathrm{C}$, respectively. Typically, $\mathrm{GaAs}(001)$ substrates are used, on which lattice-matched $\mathrm{Ga}_{0.51} \mathrm{In}_{0.49} \mathrm{P}$ buffer layers are deposited prior to island growth (see, for example, Seifert et al., 1996). OMCVDgrown islands are larger and exhibit an anisotropic shape, elongated along the [110] direction. Well developed side facets consist of $\{110\}$ and $\{111\}$ planes. The islands are truncated with a top (001) facet. Typical dimensions are $40-50 \mathrm{~nm}$ at the base along the [110] direction, and 55-65 $\mathrm{nm}$ along the [110] direction, with heights ranging from 12 to $18 \mathrm{~nm}$ (Carlsson et al., 1998). The smaller MBE-grown islands have a side facet angle of $26^{\circ}$, a base diameter of roughly $25 \mathrm{~nm}$ along the [110] 
direction, and a height of about $5 \mathrm{~nm}$. The shape and size can be strongly influenced by the growth parameters: deposition of about 3 ML's at low growth rates of $0.01 \mathrm{ML} / \mathrm{s}$ leads to somewhat larger islands in $\mathrm{MBE}$ with heights around $8 \mathrm{~nm}$ and photoluminescence emission around $1.7 \mathrm{eV}$, compared to emission above $1.8 \mathrm{eV}$ for standard growth rates of $0.5 \mathrm{ML} / \mathrm{s}$.

Local strain fields of InP dots embedded in GaInP were studied by Jin-Phillip (2000). A strong dependence of the strain fields on the spacer-layer thickness was deduced from TEM data, in particular for stacked InP dots as used for lasers. The compressive strain within the dots decreased with decreasing spacing between the layers, which explains the observed increasing redshift in photoluminescence.

The different elastic relaxation along the two orthogonal $\langle 110\rangle$ directions was observed in XRD experiments by Schmidbauer, Hatami, et al. (2002), who correlated the structural results with the polarization dependence of photoluminescence data. However, as pointed out by Schmidt, Manz, and Eberl (2002), a pronounced polarization dependence of the same magnitude and sign as for the InP islands is observed already in the GaInP matrix, due to its inherent anisotropy.

The optical properties of neutral and charged small InP dots were investigated in detail using photoluminescence by Persson et al. (2003), and simulated on the basis of a $\mathbf{k} \cdot \mathbf{p}$ calculation of the energy states.

With InP dots, single-photon emission at wavelengths between 640 and $690 \mathrm{~nm}$ was recently demonstrated by Zwiller et al. (2003). Photon correlation experiments were performed at temperatures up to $50 \mathrm{~K}$. Even higher-temperature operation might be possible for larger band-gap barrier materials like AlGaInP.

\section{F. ErAs and ErSb}

Bulk ErAs crystallizes in a rocksalt lattice and is antiferromagnetic below $4 \mathrm{~K}$. Kadow et al. (2003) have grown ErAs $(1.8 \mathrm{ML}) / \mathrm{GaAs}$ superlattices at $630^{\circ} \mathrm{C}$, in which well-isolated ErAs islands with a lateral extension of about $10 \mathrm{~nm}$ form with a density of about 4 $\times 10^{11} \mathrm{~cm}^{-2}$. With decreasing growth temperature of $580^{\circ} \mathrm{C}$, the islands become smaller, with a lateral extent of $4 \mathrm{~nm}$, while the density increases to $2 \times 10^{12} \mathrm{~cm}^{-2}$. The typical island height is equivalent to 3-4 ML's. Such island superlattices are used as substitutes for lowtemperature GaAs in ultrafast photoconductors and for the generation of submillimeter waves. Recently, Hanson et al. (2003) reported the growth of self-assembled $\mathrm{ErSb}$ semimetallic nanometer-sized particles in a $\mathrm{GaSb}$ matrix, which allows for strong absorption at a wavelength of $1.55 \mu \mathrm{m}$.

\section{G. CdSe}

Lee et al. (1998), Kratzert et al. (1999, 2001), and Strassburg et al. (2000) have grown coherent islands by depositing about 3-ML CdSe with MBE at temperatures between 230 and $340{ }^{\circ} \mathrm{C}$ on $\mathrm{ZnSe}$ buffers on $\mathrm{GaAs}(001)$. Depending on growth and annealing procedures, either high-density CdSe islands with diameters less than $10 \mathrm{~nm}$ (type $A$ ) were obtained or lower-density ones with diameters of about $16 \mathrm{~nm}$ (type $B$ ). The Cd content was established from TEM images using lattice fringe analysis (Schikora et al., 2000). In photoluminescence, typical emission energies between 2.45 and $2.6 \mathrm{eV}$ were observed, where the larger type- $B$ islands dominated the room-temperature photoluminescence and the smaller, much denser type- $A$ ones the photoluminescence around $120 \mathrm{~K}$ (Strassburg et al., 2000; Jones et al., 2003). Stacked CdSe multilayers were investigated by Krestnikov et al. (1999): for ZnSe spacer-layer thicknesses below $3 \mathrm{~nm}$, vertical stacking was observed, whereas for thicknesses above $3 \mathrm{~nm}$ anticorrelation occurred, which was explained by the elastic anisotropy of the $\mathrm{ZnSe}$ spacer layers (Holý, Springholz, et al., 1999; Shchukin and Bimberg, 1999).

Semimagnetic properties are obtained if $\mathrm{Mn}$ is incorporated into CdSe islands. Kratzert et al. (2001) and Titova et al. (2002) have studied such islands, which were fabricated either by sandwiching $\mathrm{CdSe}$ between $\mathrm{ZnMnSe}$ or by directly depositing $\mathrm{CdMnSe}$ on $\mathrm{ZnSe} / \mathrm{GaAs}$ buffers, followed by an annealing step at $310^{\circ} \mathrm{C}$, which initiates island growth. Magneto-optical investigations reveal $g$ factors up to 200. From timedependent photoluminescence measurements, Seufert et al. (2002) deduced the spin response time of the magnetic ions in the exciton exchange field of the semimagnetic material. The temperature dependence of the magnetic polaron formation time was much weaker than for comparable bulk CdMnSe samples. Because of its material properties, $\mathrm{CdSe}$ submonolayer inclusions in $\mathrm{ZnSe}$ are also ideally suited for the investigation of bound polarons (see Woggon et al., 2003).

CdSe islands have superior properties for singlephoton emission as compared to InAs quantum dots (Makino et al., 2003). Due to island densities as low as $10^{9} \mathrm{dots} / \mathrm{cm}^{-2}$, single-photon emission from individual dots can be easily realized. II-VI compound quantum dots feature a much stronger exciton-biexciton splitting than InAs dots. For CdSe islands, this splitting is about $20 \mathrm{meV}$, in comparison to 1-2 $\mathrm{meV}$ for InGaAs. Consequently CdSe islands promise single-photon generation at higher temperatures. So far, however, the excitonic lines from CdSe dots around $2.4 \mathrm{eV}$ are inhomogeneously broadened up to $300 \mu \mathrm{eV}$.

\section{H. PbSe}

The self-organized growth of pyramidal $\mathrm{PbSe}$ islands on [111]-oriented PbEuTe buffer layers leads to 3D quantum dot superlattices (Springholz et al., 1998) with different island correlation regimes depending on spacer thickness: vertical, fcc-like, or uncorrelated [see Sec. II.C and Springholz, Pinczolits, et al. (2000a)]. The transitions between the different stacking regimes are in good agreement with the results of a model that de- 
scribes the interlayer dot interactions as a function of the PbSe dot size. A hitherto unparalleled control of island size, density, and uniformity has been achieved. A material quality sufficient for devices was demonstrated by the fabrication of a vertical cavity surface emitting laser (VCSEL) by Springholz, Schwarzl, et al. (2001). A different application of such IV-VI island superlattices is for thermoelectric devices (see below). While Springholz et al. use $\mathrm{BaF}_{2}$ substrates, Alchalabi et al. (2003) have grown $\mathrm{PbSe}$ islands on $\mathrm{Si}(111)$ substrates using $\mathrm{CaF}_{2}$ and $\mathrm{PbTe}$ buffer layers. An extremely small full width at half maximum (FWHM) of the island size distribution of 0.02 was reported.

\section{APPLICATIONS}

\section{A. Optical applications: Detectors and lasers}

The impact of quantum dots on detectors and lasers has been reviewed by Bimberg et al. (1999) and more recently by Grundmann (2002), Ledentsov et al. (2002), Ustinov and Zhukov (2002), Shchukin et al. (2003), and Ustinov et al. (2003).

Quantum dot detectors with genuine zerodimensional properties have been fabricated mainly from buried InAs dot layers in GaAs. In comparison to InAs-based quantum well infrared detectors (QWIP's) the quantum dot infrared detectors (QDIP's) in principle should not suffer from restrictions of the intersubband transition selection rules $\left(E_{z} \neq 0\right)$, i.e., they are sensitive to light with any incidence angle, because of carrier confinement in all three dimensions (Chen et al., 2001; Liu, Gao, et al., 2001; Brunner, 2002; Lin and Singh, 2002; Ye et al., 2002; Aslan et al., 2003). However, the quantum dot shape has a significant influence on the polarization selection rules due to the low aspect ratio. A significantly increased normal-incidence photocurrent signal is observed for vertically coupled dots in comparison to uncoupled ones, which is explained by a lowering of the $s$-like ground state and a hybridization of the $p_{x, y}$ states with the wetting-layer states (Adawi et al., 2003). With stacked InAs dot layers, a voltage-controllable multiwavelength response ranging from 5.5 to $10.9 \mu \mathrm{m}$ has been established (Ye et al., 2002). For such structures, peak detectivities at $T=77 \mathrm{~K}$ of $D^{*}=5.8$ $\times 10^{9} \mathrm{~cm} \sqrt{\mathrm{Hz}} \mathrm{W}^{-1}$ at $5.9 \mu \mathrm{m}$ have been achieved.

It was realized long ago that the quasi-zerodimensional density of states in self-organized quantum dots embedded in a larger band-gap matrix offers low threshold current densities for lasing. Furthermore a low temperature dependence of this threshold current density has been demonstrated and, most importantly, with InAs islands instead of GaInAs quantum wells longerwavelength lasers can be obtained. With these dot lasers GaAs technology might have the potential to replace InP-based lasers for wavelengths around $1.3 \mu \mathrm{m}$ and possibly even up to $1.55 \mu \mathrm{m}$. At operating temperatures up to $80^{\circ} \mathrm{C}$, these lasers seem to outperform commercial InP-based quantum well lasers. For such operating temperatures a characteristic temperature $T_{\mathrm{o}}$ of about $220 \mathrm{~K}$ over the temperature range from 0 to $81^{\circ} \mathrm{C}$ was achieved by $p$-type modulation doping of the dots (Shchekin and Deppe, 2002). For the $1.3 \mu \mathrm{m}$ lasers, two approaches are used for the growth of the islands: (i) one is based on atomic layer epitaxy which results in large uniform InAs islands of comparatively low density; (ii) the second one uses conventional molecular beam epitaxy, but the InAs dots are either buried with InGaAs prior to GaAs capping, or the islands are embedded in GaInAs or GaInAlAs quantum wells (Ledentsov et al., 2002, Maximov, 2003), so-called dot in a well (DWELL) structures. The reasons for the long-wavelength emission are the reduced dot strain, the reduced confinement and the increased dot size as compared to dots embedded directly in GaAs. Recently, Liu et al. (2004) reported a further substantially improved performance of $1.3 \mu \mathrm{m}$ multilayer quantum dot lasers by suppressing threading dislocation formation through the growth of a high temperature GaAs space layer. A three-dot-layer DWELL structure exhibits a minimum threshold current density of $13 \mathrm{~A} / \mathrm{cm}^{-2}$, a room temperature value of $33 \mathrm{~A} / \mathrm{cm}^{-2}$ with emission at $1.31 \mu \mathrm{m}$ and operation up to $105^{\circ} \mathrm{C}$. A further promising approach was reported by Sellers et al. (2003), who showed that incorporation of a thin AlInAs layer after the deposition of the InAs islands leads to the improvement of the island homogeneity and to photoemission at longer wavelengths, due to reduced In segregation and intermixing. Furthermore, the increased confinement potential for the electronic states is beneficial for laser operation.

Beside the wavelength range up to $1.55 \mu \mathrm{m}$, InAs quantum dot lasers also have a large potential for highpower operation $(6-\mathrm{W} \mathrm{cw})$ at shorter wavelengths in between 1.1 and $1.2 \mu \mathrm{m}$ (Ribbat et al., 2003). Verticalcavity surface-emitting lasers have been reported by several groups, e.g., Chen et al. (2002) and Ledentsov et al. (2002). Threshold currents below $2 \mathrm{~mA}$, operation voltages below $2 \mathrm{~V}$, and sufficiently high differential gain were reported to make such structures suitable for devices operating at $1.3 \mu \mathrm{m}$.

A review of InP-based quantum dot lasers by Schmidt et al. can be found in Grundmann (2002). Such lasers consist typically of three stacked InP dot layers sepa-

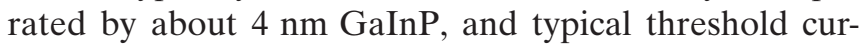
rents at room temperature are around $2.3 \mathrm{kA} / \mathrm{cm}^{2}$ with $T_{0}=42 \mathrm{~K}$.

Another interesting development is the use of AlInAs quantum dots in GaAs/AlAs cascade laser structures (Ulbrich et al., 2003). Such structures are expected to have better performance than 2D quantum well cascades due to suppressed nonradiative relaxation as well as surface normal emission.

\section{B. New concepts}

Developments and proposals in several new areas deserve mention here. 
- Positioning of islands. For device applications, uniform islands are required. One way to achieve this goal is the growth of regular arrays by a combination of lithography and self-assembly (see Sec. II.C). With the same philosophy a controlled positioning of islands is also possible. This could make new devices feasible that rely on nanostructures with highly predictable and reproducible properties, for instance, concepts for the realization of qbits as suggested by Friesen et al. (2003). A completely different approach to ordering quantum dots has been suggested by Lee et al. (2002). These authors align $\mathrm{ZnS}$ nanocrystals on a genetically engineered M13 bacteriophage-based liquid-crystal system. The bacteriophage were selected to have a specific recognition moiety for $\mathrm{ZnS}$, and when coupled with a ZnS solution precursor, they spontaneously evolve into an ordered material. The authors see a potential of their method for ordering and aligning different types of inorganic materials in 3D layered structures.

Hennessy et al. (2003) have recently embedded InAs quantum dots within a single hole defect of a square-lattice photonic crystal microcavity with an extremely high quality factor $(Q \approx 4000)$. Such structures are candidates for achieving both spectral and spatial coupling of a single quantum dot to a cavity mode for quantum electrodynamic phenomena.

- Dot-FET's. The possibility of achieving laterally ordered islands and of modifying the band structure in a capping layer through the strain fields of buried dots has led to the concept of the Dot-FET, a dotbased field-effect transistor on $\mathrm{Si}$ (Schmidt and Eberl, 2001). The misfit strains in $n$-doped $\mathrm{Si}$ above buried Ge islands lower the energy of the $\Delta_{2}$ valleys, which have a small effective transport mass, and thus significantly enhance both the electron mobility and the maximum device frequency. The necessary complementary $p$ channel is realized with strained $\mathrm{Ge}$ in the self-assembled and properly modulationdoped islands themselves.

- Thermoelectric devices. Harman et al. (2002) have found that $\mathrm{PbSeTe}$ quantum dots embedded in $\mathrm{PbTe}$ barriers in a superlattice with about 8000 periods have excellent thermoelectric figures of merit up to about $Z T=2$ at room temperature. This material combination yields a large increase in the thermoelectric Seebeck coefficient and of $Z T$ with respect to both $n$ - and $p$-type PbTe, which is attributed to a lowering of the lattice thermal conductivity, caused by the huge number of interfaces and by carrier confinement in the quantum dots, which increases the power factor (Koga et al., 1999). IV-VI compounds are advantageous because, even at high carrier concentrations, the mobilities and hence the electrical conductivities are high. According to Harman et al. (2002), a further increase of $Z T$ up to values of 3 seems feasible if instead of PbSeTe islands a quaternary system $\mathrm{PbSnSeTe}$ is used, with lower band gap and even further reduced thermal conductivity. With a $\mathrm{PbSeTe} / \mathrm{PbTe}$ quantum dot superlattice test device, cooling to about $44 \mathrm{~K}$ below room temperature has been achieved.

- Entangling of quantum states. At present, much work is being performed on optical investigations of single dots, in particular on InAs dots, as well as on coupled dots, as a first step towards solid-state-based quantum communication and computing. Coherent nonlinear spectroscopy on single quantum dots has provided a wealth of information on dephasing and energy relaxation times (Guenther et al., 2002). Combined with the possibility of precisely arranging islands, this opens the possibility for studying critical coupling effects between dots (Bayer et al., 2001). Coherent optical control of a two-exciton state in a single $\mathrm{GaAs}$ island was reported by $\mathrm{Li}, \mathrm{Wu}$, et al. (2002).

- Magnetic nanostructures. A detailed knowledge of magnetic interactions in nanostructures is considered essential both for fundamental physics and for further development of magnetic storage devices [see, for instance, Kortright et al. (1999)]. In addition, the advent of spintronics, or spin-based electronics, as a vision for future devices has also increased interest in magnetic semiconductor nanostructures (Wolf et al., 2001).

It is well known that 2D systems like epitaxial layers and multilayers exhibit magnetic properties different from those of bulk materials. The study of magnetic systems with one and zero dimensions is challenging, because with reduced dimensions fluctuations become relatively more important, and thus magnetic ordering tends to decrease (Gambardella et al., 2002). Nevertheless, for Co deposited on a vicinal Pt(997) surface, Gambardella et al., 2002 observed ferromagnetism for highdensity parallel monoatomic chains of $\mathrm{Co}$, and evidence found for long-range ordering at $T=10 \mathrm{~K}$, i.e., below the threshold temperature. The magnetic properties were studied by x-ray magnetic circular dichroism at energies corresponding to the Co $L_{2,3}$ absorption edges, in order to increase the sensitivity. The difference between two absorption spectra for left and right circularly polarized radiation characterizes the dichroism. The amplitude of the dichroic signal is a measure of the magnetization of the wire array. Using $\mathrm{x}$-ray absorption and x-ray magnetic circular dichroism, Gambardella et al. (2003) succeeded in observing the magnetic anisotropy of isolated Co atoms on $\mathrm{Pt}(111)$ surfaces. This anisotropy is due to a symmetry reduction at the ordered Pt surface. Furthermore, Co nanoparticles up to 40 atoms each were assembled on the Pt surface, and studies of their magnetic anisotropy energy per Co atom revealed a strong decrease with average nanoparticle size.

In addition to magnetic absorption experiments, magnetic x-ray scattering can be performed. Using this method, the magnetic roughness as opposed to the usual 
structural roughness can be observed (see MacKay et al., 1996; Stepanov and Sinha, 2000).

Metallic nanostructures are already being used in devices. The giant magnetoresistance observed in artificial thin-film materials with alternating ferromagnetic and nonmagnetic layers has found widespread applications in magnetic hard drives. Using spin-polarized STM, it has been possible to study the spin structure in the vortex cores of $\mathrm{Fe}$ islands (average height about 8-9 $\mathrm{nm}$ ) deposited on single crystalline tungsten substrates (Wachowiak et al., 2002).

Nanostructures based on magnetic semiconductors are so far of less impact. Chye et al. (2002) recently demonstrated spin injection from a GaMnAs layer into InAs islands. An estimated spin-injection efficiency of $70 \%$ was reported by Seufert et al. (2004), who used a $\mathrm{BeMnZnSe}$ spin aligner to inject into CdSe islands.

A different approach to tunable magnetic nanostructures is the use of colloidal crystals, which consist of crystalline inorganic cores coordinated by organic monolayers. 3D binary superlattices with tunable distance and tunable size of the magnetic nanocrystals (Co and $\mathrm{Fe}$ compounds) were synthesized by Murray et al. (2001) and Redl et al. (2003).

- Semiconductor nanowires Recently, high-quality semiconductor nanowires have been grown on prepatterned substrates through a vapor-liquid-solid growth mode (Ohlsson et al., 2001). Using prefabricated catalytic nanoparticles, e.g., size-selected gold aerosol particles, this group grew $2 \mathrm{D}$ arrays of vertically oriented nanowires of various materials $(\mathrm{Si} / \mathrm{Ge}$, InAs/InP, etc.) epitaxially. The wires can be fabricated by chemical-beam epitaxy, by OMCVD, and by solid-source MBE. Altering the chemical composition along the wires allows one to realize zerodimensional structures, i.e., quantum dots, which are contacted by quantum wires (Björk et al., 2002; Gudiksen et al., 2002; Samuelson, 2003). Typical wire diameters of between 8 and $100 \mathrm{~nm}$ were reported. Due to their comparatively small diameter, wire heterostructures can undergo lateral relaxation. The nanowires and embedded central nanodots allow for the study of quantum confinement phenomena, for Coulomb-blockade studies, for single-electron transistors, and for single-photon sources. Whereas the natural accumulation layer on InAs does not require additional protection, for some materials such as $\mathrm{GaAs}$ the core has to be embedded in a proper shell material (Lauhon et al., 2002). With 2D arrays of nanowires, photonic crystals can be fabricated in a bottom-up procedure.

\section{OUTLOOK AND PROSPECTS}

Considerable progress has been achieved in the past decade in the refinement of fabrication methods for selfassembled nanostructures. This imposes the need for a further improvement in characterization methods. In particular, the composition distribution within nano- structures has to be measured precisely in all three dimensions. Whereas several techniques have been used to determine the vertical composition gradients within islands, precise information on lateral composition gradients is still largely lacking. Results have been obtained from TEM and STM, but they are not sufficient to reliably establish the 3D distribution of chemical composition in a nanostructure. X-ray diffraction suffers in this respect from the fact that it investigates the statistical average over many islands, which smears out the fine details of the composition and strain distribution.

Nevertheless, $x$-ray scattering techniques have gained importance due to the abilities offered by thirdgeneration synchrotron sources. For the determination of lateral composition gradients, measurements with high real space resolution have been suggested (DiFonzo et al., 2000). X-ray beams focused to less than 100-nm spot sizes will be required. Such submicron focussing instrumentation is currently being developed at synchrotron radiation sources, which offer the required brightness for such experiments. In addition, the tunability of photon energies is advantageous, as anomalous scattering can be exploited to enhance contrast.

With small beams, it is also possible to illuminate small parts of a sample coherently. Advances in computational methods have made it possible to invert corresponding diffraction patterns uniquely to a real-space image (Miao et al., 1999; Robinson et al., 2001; Williams et al., 2003). Vartanyants and Robinson (2003) have suggested instead of illuminating one particular nanostructure, rather illuminating coherently a finite array of quantum dots. For successful reconstruction the diffraction pattern has to be oversampled in a sufficiently large reciprocal-space range $\Delta Q>2 \pi / L$, where $L$ denotes the average island size. Vartanyants and Robinson (2003) have recently developed an algorithm which they deem to be capable of reconstructing not only the shape, but also the strain profile, exploiting the asymmetry of diffraction patterns.

With MBE or chemical vapor deposition growth chambers attached to dedicated beam lines at synchrotron sources (Jenichen et al., 2003), new insight into the dynamics of growth phenomena can be obtained. Renaud et al. (2003) have achieved in situ monitoring of the evolving nanoparticle size and shape for the growth of $\mathrm{Pd}$ islands on $\mathrm{MgO}$, and $\mathrm{Co}$ nanodots on $\mathrm{Au}(111)$ surfaces. Reciprocal-space maps were recorded in GISAXS geometry with a two-dimensional chargecoupled device detector, enabling the collection of data for one map in a few tenths down to hundredths of a second. This technique makes real-time measurements for growth or annealing studies possible. As GISAXS yields information on buried interfaces as well, this technique is also well suited to monitor size and shape changes during capping. The potential of time-resolved in situ surface $\mathrm{x}$-ray diffraction studies has been demonstrated by Kaganer et al. (2003), who observed the coarsening kinetics of $\beta(2 \times 4)$ reconstruction domains on $\operatorname{GaAs}(001)$ surfaces.

The studies described above show that, with self- 
organized semiconductor nanostructures, impressive advances have been achieved on the concepts of carrier confinement. It also had become clear that further control of the formation and addressing of nanostructures is required. This goal will be difficult to achieve using selforganization alone, but a combination with other techniques may be successful. We believe that three routes are most promising: (i) a combination with rather conventional lithography will allow for controlled positioning of nanostructures without losing their inherent advantages; (ii) seeded growth with catalytic nanoparticles will allow for structures significantly smaller than Stranski-Krastanow islands; (iii) combining selfassembly with the techniques of organic and biochemistry, the controlled fabrication of extremely small structures (few $\mathrm{nm}$ in all three dimensions) and their selective addressing seems feasible.

\section{Note added in proof}

After finishing this manuscript two reviews appeared: (i) Skolnick, M.S. and D.J. Mowbray, 2004, on fundamental physics and device applications of self-assembled semiconductor quantum dots, focusing on optical properties of InGaAs quantum dots and on quantum dot lasers and detectors; (ii) Schmidbauer, M., 2004, which reviews $\mathrm{x}$-ray diffuse scattering from self-organized mesoscopic semiconductor structures.

\section{ACKNOWLEDGMENTS}

We would like to thank our co-workers and colleagues G. Chen, A. A. Darhuber, T. Fromherz, A. Hesse, I. Kegel, R. T. Lechner, M. Meduña, T. H. Metzger, M. Mühlberger, J. Novak, M. Pinczolits, A. Raab, T. Roch, F. Schäffler, C. Schelling, T. U. Schülli, G. Springholz, and Zhenyang Zhong for their contributions, as well as all authors who provided figures. This work was supported by Fonds zur Förderung der wissenschaftlichen Forschung (FWF) Vienna Grant No. 14684; Gesellschaft für Mikroelectronik (GMe), Vienna; European Commission (EC), Brussels (HPRN-1999-00123 and HPRI-CT1999-00040/2001-00140); and by the Grant Agency of the Czech Republic (202/03/0148).

\section{REFERENCES}

Adawi, A. M., E. A. Zibik, L. R. Wilson, A. Lemaitre, J. W. Cockburn, M. S. Skolnick, M. Hopkinson, G. Hill, S. L. Liew, and A. G. Cullis, 2003, Appl. Phys. Lett. 82, 3415.

Adelmann, C., J. Simon, G. Feuillet, N. T. Pelekanos, B. Daudin, and G. Fishmann, 2000, Appl. Phys. Lett. 76, 1570.

Ahrenkiel, S. P., A. G. Norman, M. M. Al-Jassim, A. Mascarenhas, J. Mirecki-Millunchick, R. D. Twesten, S. R. Lee, D. M. Follstaedt, and E. D. Jones, 1998, J. Appl. Phys. 84, 6088.

Alchalabi, K., D. Zimin, G. Kostorz, and H. Zogg, 2003, Phys. Rev. Lett. 90, 026104.

Alerhand, O. L., D. Vanderbilt, R. D. Meade, and J. D. Joannopoulos, 1988, Phys. Rev. Lett. 61, 1973.
Amar, J. G., and F. Family, 1995, Phys. Rev. Lett. 74, 2066. Andreev, A. D., J. R. Downes, D. A. Faux, E. P. O'Reilly, 1999, J. Appl. Phys. 86, 297.

Asaro, R. J., and W. A. Tiller, 1972, Metall. Trans. 3, 1789.

Aslan, B., H. C. Liu, M. Korkusinski, S. J. Cheng, and P. Hawrylak, 2003, Appl. Phys. Lett. 82, 630.

Bahr, D., W. Press, R. Jebasinski, and S. Mantl, 1990, Phys. Rev. B 41, 5500.

Barabash, R. I., and M. A. Krivoglaz, 1987, Sov. Phys. Solid State 29, 1768.

Barker, J. A., and E. P. O'Reilly, 2000, Phys. Rev. B 61, 13840. Baskes, M. I., 1987, Phys. Rev. Lett. 59, 2666.

Bauer, E., M. Mundschau, and W. Swiech, 1991, J. Vac. Sci. Technol. B 9, 403.

Bayer, M., 2002, Nature (London) 418, 597.

Bayer, M., P. Hawrylak, K. Hinzer, S. Fafard, M. Korkusinski, Z. R. Wasilewski, O. Stern, and A. Forchel, 2001, Science 291, 451.

Bell, G. R., T. J. Krzyzewski, P. B. Joyce, and T. S. Jones, 2000, Phys. Rev. B 61 R10551.

Benabbas, T., Y. Androussi, and A. Lefebvre, 1999, J. Appl. Phys. 86, 1945.

Bester, G., S. Nair, and A. Zunger, 2003, Phys. Rev. B 67, 161306(R).

Bester, G., and A. Zunger, 2003, Phys. Rev. B 68, 073309.

Biasiol, G., A. Gustafsson, K. Leifer, and E. Kapon, 2002, Phys. Rev. B 65, 205306.

Bimberg, D., M. Grundmann, and N. N. Ledentsov, 1999, Quantum Dot Heterostructures (Wiley, New York).

Björk, M. T., B. J. Ohlsson, T. Sass, A. I. Persson, C. Thelander, M. H. Magnusson, K. Deppert, L. R. Wallenberg, and L. Samuelson, 2002, Appl. Phys. Lett. 80, 1058.

Boscherini, F., G. Capellini, L. DiGaspare, F. Rosei, N. Motta, and S. Mobilio, 2000, Appl. Phys. Lett. 76, 682.

Brandt, O., L. Tapfer, K. H. Ploog, R. Bierwolf, M. Hohenstein, F. Phillip, H. Lage, A. Heberle, 1991, Phys. Rev. B 44, 8043 .

Brault, J., J. S. Tanaka, E. Sarigiannidou, J. L. Rouvière, B. Daudin, G. Feuillet, and H. Nakagawa, 2003, J. Appl. Phys. 93, 3108.

Browning, N. D., I. Arslan, P. Moeck, T. Topuria, 2001, Phys. Status Solidi B 227, 229.

Bruls, D. M., P. M. Koenraad, M. Hopkinson, J. H. Wolter, and H. Salemink, 2002, Appl. Surf. Sci. 190, 258.

Bruls, D. M., P. M. Koenraad, H. W. M. Salemink, J. H. Wolter, M. Hopkinson, M. S. Skolnick, 2003, Appl. Phys. Lett. 82, 3758.

Bruls, D. M., J. Vugs, P. Koenraad, H. Salemink, J. H. Wolter, M. Hopkinson, M. Skolnick, F. Long, and S. P. A. Gill, 2002, J. Appl. Phys. 81, 1708.

Brunhes, T., P. Boucaud, S. Sauvage, F. Aniel, J. M. Lourtioz, C. Hernandez, Y. Campidelli, O. Kermarrec, D. Bensahel, G. Faini, and I. Sanges, 2000 Appl. Phys. Lett. 77, 1822.

Brunner, K., 2002, Rep. Prog. Phys. 65, 27.

Brunner, K., J. Zhu, C. Miesner, G. Abstreiter, O. Kienzle, and F. Ernst, 2000, Physica E (Amsterdam) 7, 881.

Buda, F., J. Kohanoff, and M. Parinello, 1992, Phys. Rev. Lett. 69, 1272.

Budiman, R. A., and H. E. Ruda, 2000, J. Appl. Phys. 88, 4586. Burton, W. K., N. Cabrera, and F. C. Frank, 1951, Philos. Trans. R. Soc. London, Ser. A 243, 299.

Capellini, G., M. D. Seta, F. Evangelisti, 2001, Appl. Phys. Lett. 78, 303. 
Carlsson, A., L. R. Wallenberg, C. Persson, and W. Seifert, 1998, Surf. Sci. 406, 48.

Cazayous, M., J. Groenen, F. Demangeot, R. Sirvin, M. Caumont, T. Remmele, M. Albrecht, S. Christiansen, M. Becker, H. P. Strunk, and H. Wawra, 2002, J. Appl. Phys. 91, 6772.

Cazayous, M., J. Groenen, J. R. Huntzinger, A. Mlayah, and O. Schmidt, 2001, Phys. Rev. B 64, 033306.

Chamard, V., T. H. Metzger, A. Bellet-Amalric, B. Daudin, C. Adelmann, H. Mariette, and G. Mula, 2001, Appl. Phys. Lett. 79, 1871.

Chamard, V., T. H. Metzger, M. Stzucki, V. Holý, M. Tolan, E. Bellet-Amalric, C. Adelmann, B. Daudin, and H. Mariette, 2003, Europhys. Lett. 63, 268.

Chaparro, S. A., J. Drucker, Y. Zhang, D. Chandrasekhar, M. R. McCartney, and D. J. Smith, 1999, Phys. Rev. Lett. 83, 1199.

Chaparro, S. A., Y. Zhang, and J. Drucker, 2000a, Appl. Phys. Lett. 76, 3534.

Chaparro, S. A., Y. Zhang, and J. Drucker, 2000b, J. Appl. Phys. 87, 2245.

Charmers, S. A., J. Y. Tsao, and A. C. Gossard, 1993, J. Appl. Phys. 73, 7351.

Chason, E., M. B. Sinclair, J. A. Floro, J. A. Hunter, and R. Q. Hwang, 1998, Appl. Phys. Lett. 72, 3276.

Chen, G., N. H. Bonadeo, D. G. Steel, D. Gammon, D. S. Katzer, D. Park, and L. J. Sham, 2000, Science 289, 1906.

Chen, H., Z. Zou, C. Cao, and D. G. Deppe, 2002, Appl. Phys. Lett. 80, 350.

Chen, K. M., D. E. Jesson, S. J. Pennycook, M. Mostoler, T. Kaplan, T. Thundat, and R. J. Warmack, 1995, Phys. Rev. Lett. 75, 1582.

Chen, Z., O. Baklenov, E. T. Kim, I. Mukhametzhanov, J. Tie, A. Madhukar, Z. Ye, and J. C. Campbell, 2001, J. Appl. Phys. 89, 4558.

Chiu, C., 1999, Appl. Phys. Lett. 75, 3473.

Cho, Y. H., B. J. Kwon, J. Barjon, J. Brault, B. Daudin, H. Mariette, and L. S. Dang, 2003, Appl. Phys. Lett. 81, 4934.

Chye, Y., M. E. White, E. Johnston-Halperin, B. D. Gerardot, and D. D. Awschalom, and P. M. Petroff, 2002, Phys. Rev. B 66, 201301.

Cullis, A., D. Norris, T. Walther, M. Migliorato, and M. Hopkinson, 2002, Phys. Rev. B 66, 081305(R).

Darhuber, A. A., P. Schittenhelm, V. Holý, J. Stangl, G. Bauer, A. Abstreiter, 1997, Phys. Rev. B 55, 15652.

Daruka, I., and A. L. Barabasi, 1997, Phys. Rev. Lett. 79, 3709.

Daruka, I., and A. L. Barabasi, 1998, Appl. Phys. Lett. 72, 2102.

Daruka, I., A. L. Barabasi, S. J. Zhou, T. C. German, P. S. Lomdahl, and A. R. Bishop, 1999, Phys. Rev. B 60, R1250.

Daruka, I., and J. Tersoff, 2002, Phys. Rev. B 66, 132104.

Daruka, I., J. Tersoff, and A. L. Barabasi, 1999, Phys. Rev. Lett. 82, 2753.

Dederichs, P. H., and G. Leibfried, 1969, Phys. Rev. 188, 1175.

Dederichs, P. H., and J. Pollmann, 1972, Z. Phys. 255, 315.

Denker, U., M. Stoffel, and O. Schmidt, 2003a, Phys. Rev. Lett. 90, 196102.

Denker, U., M. Stoffel, O. G. Schmidt, and H. Sigg, 2003b, Appl. Phys. Lett. 82, 454.

DiFonzo, S., W. Jark, S. Lagomarsino, C. Giannini, L. DeCaro, A. Cedola, M. Mueller, 2000, Nature (London) 403, 638.

Dodson, B. W., and J. Y. Tsao, 1987, Appl. Phys. Lett. 51, 1325.

Dorsch, W., H. P. Strunk, H. Wawra, G. Wagner, J. Groenen, and R. Carles, 1998, Appl. Phys. Lett. 72, 179.
Dunbar, A., U. Bangert, P. Dawson, M. Halsall, Y. Shiraki, M. Miura, I. Berbezier, B. Joyce, and J. Zhang, 2001, Phys. Status Solidi B 224, 265.

Dunbar, A., M. Halsall, P. Dawson, U. Bangert, M. Miura, and Y. Shiraki, 2001, Appl. Phys. Lett. 78, 1658.

Duport, C., P. Nozières, and J. Villain, 1995, Phys. Rev. Lett. 74, 134.

Dwir, B., K. Leifer, and E. Kapon, 2003, Phys. Rev. B 67, 075302.

Eaglesham, D. J., and M. Cerullo, 1990, Phys. Rev. Lett. 64, 1943.

Ebbsjoe, I., R. K. Kalia, A. Nakano, J. P. Rino, and P. Vashishta, 2000, J. Appl. Phys. 87, 7708.

Ebiko, Y., S. Muto, D. Suzuki, S. Itoh, K. Shiramine, T. Haga, Y. Nakata, and N. Yokohama, 1998, Phys. Rev. Lett. 80, 2650. Ebiko, Y., S. Muto, D. Suzuki, S. Itoh, H. Yamakoshi, K. Shiramine, T. Haga, K. Unno, and M. Ikeda, 1999, Phys. Rev. B 60, 8234.

Eisele, H., O. Flebbe, T. Kalka, C. Preinesberger, F. Heinrichsdorff, A. Krost, D. Bimberg, and M. Dähne-Prietsch, 1999, J. Appl. Phys. 75, 106.

Eisenberg, H. R., and D. Kandel, 2002, Phys. Rev. B 66, 155429.

Faleev, N., K. Pavlov, M. Tabuchi, and Y. Takeda, 1999, Jpn. J. Appl. Phys., Part 1 38, 188.

Faux, D. A., 1994, J. Appl. Phys. 75, 186.

Faux, D. A., and G. S. Pearson, 2000, Phys. Rev. B 62, R4798. Finley, J. E., M. Sabathil, R. Oulton, A. I. Tartakovskii, D. J. Mowbray, M. S. Skolnick, S. Liew, M. Hopkinson, and P. Vogl, 2004, Photonics Spectra 21, 1999.

Flocken, J. W., and J. R. Hardy, 1970, Phys. Rev. B 1, 2447.

Floro, J. A., E. Chason, M. B. Sinclair, L. B. Freund, and G. A. Lucadamo, 1998, Appl. Phys. Lett. 73, 951.

Floro, J. A., E. Chason, R. D. Twesten, R. Q. Hwang, and L. B. Freund, 1997, Phys. Rev. Lett. 79, 3946.

Floro, J. A., M. B. Sinclair, E. Chason, L. B. Freund, R. D. Twesten, R. Q. Hwang, and G. A. Lucadamo, 2000, Phys. Rev. Lett. 84, 701.

Follstaedt, D. M., J. L. Reno, E. D. Jones, S. R. Lee, A. G. Norman, H. R. Moutinho, A. Mascarenhas, and R. D. Twesten, 2000, Appl. Phys. Lett. 77, 669.

Follstaedt, D. M., R. D. Twesten, J. M. Millunchick, S. R. Lee, E. D. Jones, S. P. Ahrenkiel, Y. Zhang, A. Mascarenhas, 1998, Physica E (Amsterdam) 2, 325.

Friesen, M., M. P. Rugheimer, D. E. Savage, M. G. Lagally, D. W. van der Weide, R. Joynt, and M. A. Eriksson, 2003, Phys. Rev. B 67, 121301 (R).

Fry, P. W., I. E. Itskevich, D. J. Mowbray, M. S. Skolnick, J. J. Finley, J. A. Barker, E. P. O'Reilly, L. R. Wilson, I. A. Larkin, P. A. Maksym, M. Hopkinson, M. Al- Khafaji, et al., 2000, Phys. Rev. Lett. 84, 733.

Fry, P. W., I. E. Itskevich, S. R. Parnell, J. J. Finley, L. R. Wilson, K. L. Schumacher, D. J. Mowbray, M. S. Skolnick, M. Al-Khafaji, A. G. Cullis, M. Hopkinson, J. C. Clark, and G. Hill, 2000, Phys. Rev. B 62, 16784.

Gambardella, P., A. Dallmeyer, K. Maiti, M. C. Malagoli, W. Eberhardt, K. Kern, and C. Carbone, 2002, Nature (London) 416, 301.

Gambardella, P., S. Rusponi, M. Veronese, S. S. Dhesi, C. Grazioli, A. Dallmeyer, I. Cabria, R. Zeller, P. H. Dederichs, K. Kern, C. Carbone, and H. Brune, 2003, Science 300, 1130. Gammon, D., and D. G. Steele, 2002, Phys. Today 55 (10), 36. Gao, H., 1994, J. Mech. Phys. Solids 42, 741. 
Gerard, J. M., and B. Gayral, 1999, J. Lightwave Technol. 17, 2089.

Glas, F., 1991, J. Appl. Phys. 70, 3556.

Glas, F., 2000, Phys. Rev. B 62, 7393.

Glas, F., 2001, J. Appl. Phys. 90, 3232.

Glas, F., 2002, Appl. Surf. Sci. 188, 9.

Gleize, J., F. Demangeot, J. Frandon, M. A. Renucci, M. Kuball, B. Damilano, N. Grandjean, and J. Massies, 2001, Appl. Phys. Lett. 79, 686.

Goldstein, L., F. Glas, J. Y. Marczin, M. N. Charasse, and G. LeRoux, 1985, Appl. Phys. Lett. 47, 1099.

González, J. C., R. Magalhaes-Paniago, W. N. Rodrigues, A. Malachias, I. Mazzaro, C. Cusatis, T. H. Metzger, and J. Peisl, 2001, Appl. Phys. Lett. 78, 1056.

González, L., J. M. Garcia, R. Garcia, F. Briones, J. MartinezPastor, and C. Ballesteros, 2000, Appl. Phys. Lett. 76, 1104.

Grandidier, B., Y. M. Niquet, B. Legrand, J. P. Nys, C. Priester, D. Stiévenard, J. M. Gérard, and V. Thierry-Mieg, 2000, Phys. Rev. Lett. 85, 1068.

Grenier, S., M. G. Proietti, H. Renevier, L. Gonzalez, J. M. Garcia, and J. Garcia, 2002, Europhys. Lett. 57, 499.

Grigoriev, D., M. Hanke, M. Schmidbauer, P. Schfer, O. Konovalov, and R. Köhler, 2003, J. Phys. D 36, A225.

Grinfeld, M. A., 1986, Sov. Phys. Dokl. 31, 831.

Groenen, J., C. Priester, and R. Carles, 1999, Phys. Rev. B 60, 16013.

Grundmann, M., 2002, Ed., Nano-Optoelectronics: Concepts, Physics, and Devices (Springer, Berlin).

Grundmann, M., N. N. Ledentsov, N. Kirstaedter, F. Heinrichsdorff, A. Krost, D. Bimberg, A. O. Kosogov, S. S. Ruvimov, P. Werner, V. M. Ustinov, P. S. Kopev, and Z. I. Alferov, 1998, Thin Solid Films 318, 83.

Gudiksen, M. S., L. J. Lauhon, J. Wang, D. C. Smith, and C. M. Lieber, 2002, Nature (London) 415, 617.

Guenther, T., C. Lienau, T. Elsaesser, M. Glanemann, V. M. Axt, T. Kuhn, S. Eshlaghi, and A. D. Wieck 2002, Phys. Rev. Lett. 89, 057401.

Guest, J. R., T. H. Stievater, G. Chen, E. A. Tabak, B. G. Orr, D. G. Steel, D. Gammon, and D. S. Katzer, 2001, Science 293, 2224.

Guffarth, F., R. Heitz, A. Schliwa, O. Stier, N. N. Ledentsov, A. R. Kovsh, V. M. Ustinov, and D. Bimberg, 2001, Phys. Rev. B 64, 08530.

Guha, S., A. Madhukar, and K. C. Rajkumar, 1990, Appl. Phys. Lett. 57, 2110.

Guyer, J. E., and P. W. Voorhees, 1996, Phys. Rev. B 54, 11710.

Hadjisavvas, G., P. Sonnet, and P. C. Kelires, 2003, Phys. Rev. B 67, 241302(R).

Hanson, M. P., D. C. Driscoll, C. Kadow, and A. C. Gossard, 2003, Appl. Phys. Lett. 84, 221.

Harman, T. C., P. J. Taylor, M. P. Walsh, B. E. LaForge, 2002, Science 297, 2229.

Headrick, R. L., and J.-M. Baribeau, 1993, Phys. Rev. B 48, 9174.

Heidemeyer, H., U. Denker, C. Mueller, and O. Schmidt, 2003, Phys. Rev. Lett. 91, 196103.

Heidemeyer, H., S. Kiravittaya, C. Mueller, N. Y. Jin-Phillipp, and O. G. Schmidt, 2002, Appl. Phys. Lett. 80, 1544.

Hennessy, K., C. Reese, A. Badolato, C. F. Wang, A. Imamoglu, P. M. Petroff, E. Hu, G. Jin, S. Shi, and D. W. Prather, 2003, Appl. Phys. Lett. 83, 3650.

Henstrom, W. L., C.-P. Liu, J. M. Gibson, T. I. Kamins, and R. S. Williams, 2000, Appl. Phys. Lett. 77, 1623.
Hesse, A., J. Stangl, V. Holý, T. Roch, G. Bauer, O. G. Schmidt, U. Denker, B. Struth, 2002, Phys. Rev. B 66, 085321. Holm, M., M.-E. Pistol, and C. Pryor, 2002, J. Appl. Phys. 92, 932.

Holý, V., A. A. Darhuber, J. Stangl, G. Bauer, J. Nützel, and G. Abstreiter, 1998a, Phys. Rev. B 57, 12435.

Holý, V., A. A. Darhuber, J. Stangl, G. Bauer, J. Nützel, G. Abstreiter, 1998b, Semicond. Sci. Technol. 13, 590.

Holý, V., A. A. Darhuber, J. Stangl, S. Zerlauth, F. Schäffler, G. Bauer, N. Darowski, D. Luebbert, U. Pietsch, and I. Vavra, 1998c, Phys. Rev. B 58, 7934.

Holý, V., G. Springholz, M. Pinczolits, and G. Bauer, 1999, Phys. Rev. Lett. 83, 356.

Holý, V., J. Stangl, G. Springholz, M. Pinczolits, and G. Bauer, 2001, J. Phys. D 34, A1.

Holý, V., J. Stangl, S. Zerlauth, G. Bauer, N. Darowski, D. Luebbert, and U. Pietsch, 1999, J. Phys. D 32, A234.

Hu, C. W., A. Bell, L. Shi, F. A. Ponce, D. J. Smith, I. S. T. Tsong, 2003, Appl. Phys. Lett. 82, 2889.

Hu, S. M., 1989, J. Appl. Phys. 66, 2741.

Husberg, O., A. Khartchenko, D. J. As, H. Vogelsang, T. Frey, D. Schikora, K. Lischka, O. C. Noriega, A. Tabata, and J. R. Leite, 2001, Appl. Phys. Lett. 79, 1243.

Jacobs, S. E., M. Kemerink, P. M. Koenraad, M. Hopkinson, H. W. M. Samelink, and J. H. Wolter, 2003, Appl. Phys. Lett. 83, 290.

James, E., and N. Browning, 1998, Ultramicroscopy 78, 125.

Jenichen, B., W. Braun, V. M. Kaganer, A. G. Shtukenberg, L. Daeweritz, C.-G. Schulz, K. H. Ploog, and A. Erko, 2003, Rev. Sci. Instrum. 74, 1267.

Jesson, D. E., K. M. Chen, S. J. Pennycook, T. Thundat, and R. J. Warmack, 1995, Science 268, 1161.

Jesson, D. E., M. Kaestner, and B. Voigtländer, 2000, Phys. Rev. Lett. 84, 330.

Jesson, D. E., T. P. Munt, V. A. Shchukin, and D. Bimberg, 2004, Phys. Rev. B 69, 041302(R).

Jesson, D. E., S. J. Pennycook, J.-M. Baribeau, and D. C. Houghton, 1993a, Phys. Rev. Lett. 71, 1744.

Jesson, D. E., S. J. Pennycook, J. Z. Tischler, and J. D. Budai, 1993b, Phys. Rev. Lett. 70, 2293.

Jin, G., J. L. Liu, and K. L. Wang, 2000, Appl. Phys. Lett. 76, 3591.

Jin, G., J. L. Liu, and K. L. Wang, 2003, Appl. Phys. Lett. 83, 2847.

Jin-Phillip, N. Y., and F. Phillip, 2000, J. Appl. Phys. 88, 710.

Jogai, B., 2000, J. Appl. Phys. 88, 5050.

Jogai, B., 2001, J. Appl. Phys. 90, 699.

Jogai, B., 2002, J. Vac. Sci. Technol. B 20, 544.

Johansson, J., and W. Seifert, 2002, J. Cryst. Growth 234, 139.

Jones, D. E., J. P. Pelz, Y. H. Xie, P. J. Silverman, and G. H. Gilmer, 1995, Phys. Rev. Lett. 75, 1570.

Jones, R. A., J. M. Yarrison-Rice, L. M. Smith, H. E. Jackson, M. Dobrowolska, and J. K. Furdyna, 2003, Phys. Rev. B 68, 125333.

Joyce, B., 2003, private communication.

Kadow, C., J. A. Johnson, K. Kolstad, and A. C. Gossard, 2003, J. Vac. Sci. Technol. B 21, 29.

Kaganer, V. M., V. Braun, B. Jenichen, L. Daeweritz, and K. H. Ploog, 2003, Phys. Rev. Lett. 90, 016101.

Kaganer, V. M., and K. H. Ploog, 2001, Phys. Rev. B 64, 205301.

Kamenev, B. V., H. Grebel, L. Tsybeskov, T. I. Kamins, R. S. Williams, J. M. Baribeau, and D. J. Lockwood, 2003, Appl. 
Phys. Lett. 83, 5035.

Kamins, T. I., G. Medeiros-Ribeiro, D. A. A. Ohlberg, R. S. Williams, 1999, J. Appl. Phys. 85, 1159.

Kamins, T. I., and R. S. Williams, 1997, Appl. Phys. Lett. 71, 1201.

Kandel, D., and J. D. Weeks, 1995, Phys. Rev. Lett. 74, 3632. Kästner, M., and B. Voigtländer, 1999, Phys. Rev. Lett. 82, 2745 .

Kawamura, M., N. Paul, V. Cherepanov, and B. Voigtlaender, 2003, Phys. Rev. Lett. 91, 096102.

Keating, P. N., 1966, Phys. Rev. 145, 637.

Kegel, I., T. H. Metzger, A. Lorke, J. Peisl, J. Stangl, G. Bauer, J. M. Garcia, and P. M. Petroff, 2000, Phys. Rev. Lett. 85, 1694.

Kegel, I., T. H. Metzger, A. Lorke, J. Peisl, J. Stangl, G. Bauer, K. Nordlund, W. V. Schoenfeld, and P. M. Petroff, 2001, Phys. Rev. B 63035318.

Kegel, I., T. H. Metzger, J. Peisl, P. Schittenhelm, and G. Abstreiter, 1999a, Appl. Phys. Lett. 74, 2978.

Kegel, I., T. H. Metzger, J. Peisl, J. Stangl, G. Bauer, and D. Smilgies, 1999b, Phys. Rev. B 60, 2516.

Kelires, P. C., 1998, Int. J. Mod. Phys. C 9, 357.

Keyes, R. W., 1962, J. Appl. Phys. 33, 3371.

Kienzle, O., F. Ernst, M. Ruehle, O. G. Schmidt, and K. Eberl, 1999, Appl. Phys. Lett. 74, 269.

Kikuchi, Y., H. Sugii, and K. Shintani, 2001, J. Appl. Phys. 89, 1191.

Kim, C. S., M. Kim, J. K. Furdyna, M. Dobrowolska, S. Lee, H. Rho, L. M. Smith, H. E. Jackson, E. M. James, Y. Xin, and N. D. Browning, 2000, Phys. Rev. Lett. 85, 1124.

Kim, E. S., N. Usami, and Y. Shiraki, 1998, Appl. Phys. Lett. 92, 1617.

Kisielowski, C., P. Schwander, F. H. Bauman, M. Seibt, Y. Kim, and A Ourmazd, 1995, Ultramicroscopy 58, 131.

Koch, R., G. Wedler, J. J. Schulz, and B. Wassermann, 2001, Phys. Rev. Lett. 87, 136104.

Koga, T., T. C. Harman, S. B. Cronin, and M. S. Dresselhaus, 1999, Phys. Rev. B 60, 14286.

Kolobov, A. V., H. Oynagi, S. Wei, K. Brunner, G. Abstreiter, and K. Tanaka, 2002, Phys. Rev. B 66, 075319.

Kolosov, O. V., M. R. Castell, C. D. Marsh, G. A. D. Briggs, T. I. Kamins, and R. S. Williams, 1998, Phys. Rev. Lett. 81, 1046.

Kondrashkina, E. A., S. A. Stepanov, R. Opitz, M. Schmidbauer, R. Köhler, R. Hey, M. Wassermeier, D. Novikov, 1997, Phys. Rev. B 56, 10469.

Konkar, A., A. Madhukar, and P. Chen, 1998, Appl. Phys. Lett. 72, 220.

Kortright, J. B., D. D. Awschalom, J. Stoehr, S. D. Bader, Y, U. Idzerda, S. S. P. Parkin, I. K. Schuller, and H.-C. Siegmann, 1999, J. Magn. Magn. Mater. 207, 7.

Kovats, Z., M. Rauscher, H. Metzger, J. Peisl, R. Paniago, H.-D. Pfannes, J. Schultze, I. Eisele, F. Boscherini, and S. Ferrer, 2000, Phys. Rev. B 62, 8223.

Kratzer P., 2004, private communication.

Kratzer, P., E. Penev, and M. Scheffler, 2002, Appl. Phys. A: Mater. Sci. Process. 75, 79.

Kratzert, P. R., J. Puls, M. Rabe, and F. Henneberger, 2001, Appl. Phys. Lett. 79, 2814.

Kratzert, P. R., M. Rabe, and F. Henneberger, 1999, Phys. Rev. Lett. 83, 239.

Krestnikov, I. L., M. Strassburg, M. Caesar, A. Hoffmann, U. W. Pohl, D. Bimberg, N. N. Ledentsov, P. S. Kopev, Zh. I. Alferov, D. Litvinov, A. Rosenauer, and D. Gehrtsen, 1999,
Phys. Rev. B 60, 8695.

Kret, S., T. Benabbas, C. Delamarre, Y. Androussi, A. Doubon, J. Y. Laval, and A. Lefebvre, 1999, J. Appl. Phys. 86, 1988.

Krivoglaz, M. A., 1991, Diffraction of X-Rays and Neutrons in Imperfect Crystals (Springer, Berlin/Heidelberg).

Kryzewski, T., T. P. Joyce, G. Bell, and T. Jones, 2001, Surf. Sci. 482-485, 891.

Krzyzewski, T., P. Joyce, G. Bell, and T. Jones, 2002a, Surf. Sci. 517, 8 .

Krzyzewski, T. J., P. B. Joyce, G. R. Bell, and T. S. Jones, 2002b, Phys. Rev. B 66, 201302(R).

Lam, P.-M., and S. Tan, 2001, Phys. Rev. B 64035321.

Lauhon, L. J., M. S. Gudiksen, D. Wang, and C. M. Lieber, 2002, Nature (London) 420, 57.

Ledentsov, N. N., D. Bimberg, V. M. Ustinov, Z. I. Alferov, and J. A. Lott, 2002, Physica E (Amsterdam) 13, 871.

Lee, H., J. A. Johnson, M. Y. He, J. S. Speck, and P. M. Petroff, 2001, Appl. Phys. Lett. 78, 105.

Lee, H., J. A. Johnson, J. S. Speck, and P. M. Petroff, 2000, J. Vac. Sci. Technol. B 18, 2193.

Lee, S., I. Daruka, C. S. Kim, A.-L. Barabasi, J. L. Merz, and J. K. Furdyna, 1998, Phys. Rev. Lett. 81, 3479.

Lee, S.-W., C. Mao, C. E. Flynn, and A. M. Belcher, 2002, Science 296, 892.

LeGoues, F. K., M. C. Reuter, J. Tersoff, M. Hammar, and R. M. Tromp, 1994, Phys. Rev. Lett. 73, 300.

Leifeld, O., A. Beyer, D. Grützmacher, and K. Kern, 2002, Phys. Rev. B 66, 125312.

Leon, R., T. J. Senden, Y. Kim, C. Jagadish, and A. Clark, 1997, Phys. Rev. Lett. 78, 4942.

Li, J. H., V. Holý, M. Meduna, S. C. Moss, A. G. Norman, A. Mascarenhas, and J. L. Reno, 2002, Phys. Rev. B 66, 115312. Li, J. H., V. Holý, Z. Zhong, J. Kulik, S. C. Moss, A. G. Norman, A. Mascarenhas, J. L. Reno, and D. M. Follstaedt, 2001, J. Appl. Phys. 78, 219.

Li, X., Y. Wu, D. Steel, D. Gammon, T. H. Stievater, D. S. Katzer, D. Park, C. Piermarocchi, and L. J. Sham, 2002, Science 301, 809.

Lian, G. D., J. Yuan, L. M. Brown, G. H. Kim, and D. A. Ritchie, 1998, Appl. Phys. Lett. 73, 49.

Liao, X. Z., J. Zou, D. J. H. Cockayne, Z. Jiang, X. Wang, and R. Leon, 2000, Appl. Phys. Lett. 77, 1304.

Liao, X. Z., J. Zou, D. J. H. Cockayne, J. Qin, Z. M. Jiang, X. Wang, and R. Leon, 1999, Phys. Rev. B 60, 15605.

Liao, X. Z., J. Zou, D. J. H. Cockayne, J. Wan, Z. M. Jiang, G. Jin, and K. L. Wang, 2002, Phys. Rev. B 65, 153306.

Lichtenburger, H., M. Mühlberger, C. Schelling, and F. Schäffler 2004, submitted to J. Cryst. Growth.

Lidorikis, E., M. E. Bachlechner, R. K. Kalia, A. Nakao, P. Vashishta, and G. Z. Voyiadjis, 2001, Phys. Rev. Lett. 87, 086104.

Lin, Y. Y., and J. Singh, 2002, J. Appl. Phys. 92, 6205.

Lita, B., R. S. Goldman, J. D. Phillips, and P. K. Bhattarchaya, 1999, Appl. Phys. Lett. 75, 2797.

Liu, C.-M., J. M. Gibson, D. G. Cahill, T. I. Kamins, D. P. Basile, and R. S. Williams, 2000, Phys. Rev. Lett. 84, 1958.

Liu, F., S. E. Davenport, H. M. Evans, and M. G. Lagally, 1999, Phys. Rev. Lett. 82, 2528.

Liu, F., and M. G. Lagally, 1997, Surf. Sci. 386, 169.

Liu, F., A. H. Li, and M. G. Lagally, 2001, Phys. Rev. Lett. 87, 126103.

Liu, F., J. Tersoff, and M. G. Lagally, 1998, Phys. Rev. Lett. 80, 
1268.

Liu H. C., M. Gao, J. McCaffrey, Z. R. Wasilewski, and S. Fafard, 2001, Appl. Phys. Lett. 78, 79.

Liu, H. Y., M. Hopkinson, C. N. Harrison, M. J. Steer, R. Frith, I. R. Sellers, D. J. Mowbray, and M. S. Skolnick, 2003, J. Appl. Phys. 93, 2931.

Liu, N., J. Tersoff, O. Baklenov, A. L. Holmes, Jr., and C. K. Shih, 2000, Phys. Rev. Lett. 84, 334.

Liu Y. H., I. R. Sellers, T. J. Badcock, D. J. Mowbray, M. S. Skolnick, K. M. Groom, M. Gutiérrez, M. Hopkinson, J. S. Nig, J. P. R. David, and R. Beanland, 2004, Appl. Phys. Lett. 85, 704.

Lu, Z.-Y, F. Liu, C.-Z. Wang, X. R. Qin, B. S. Swartzentruber, M. G. Lagally, and K.-M. Ho, 2000, Phys. Rev. Lett. 85, 5603. Lueth, H., 1998, Appl. Surf. Sci. 130-132, 855.

Lundstrom, T., W. Schoenfeld, H. Lee, and P. M. Petroff, 1999, Science 286, 2312.

MacKay, J. F., C. Teichert, D. E. Savage, and M. G. Lagally, 1996, Phys. Rev. Lett. 77, 3925.

Magalhaes-Paniago, R., G. Medeiros-Ribeiro, A. Malachias, S. Kycia, T. I. Kamins, and R. S. Williams, 2002, Phys. Rev. B 66, 245312.

Makeev, M. A., and A. Madhukar, 2001, Phys. Rev. Lett. 86, 5542 .

Makeev, M. A., and A. Madhukar, 2003, Phys. Rev. B 67 , 073201.

Makeev, M. A., W. Yu, and A. Madhukar, 2003, Phys. Rev. B 68, 195301.

Makino, T., R. André, J.-M. Gérard, R. Romestain, L. S. Dang, M. Bartels, K. Lischka, and D. Schikora, 2003, Appl. Phys. Lett. 82, 2227.

Malachias, A., S. Kycia, G. Medeiros-Ribeiro, R. MagalhaesPaniago, T. I. Kamins, and R. S. Williams, 2003, Phys. Rev. Lett. 91, 176101.

Maltezopoulos, T., A. Bolz, C. Meyer, C. Heyn, W. Hansen, M. Morgenstern, and R. Wiesendanger, 2003, Phys. Rev. Lett. 91, 196804.

Marchenko, V. I., 1981, JETP Lett. 33, 381.

Marchenko, V. I., and A. Y. Parshin, 1980, Sov. Phys. JETP 52, 129.

Marquez, J., L. Geelhaar, and K. Jacobi, 2001, Appl. Phys. Lett. 78, 2309.

Martin, R. M., 1970, Phys. Rev. B 10, 4005.

Martmez-Guerrero, E., C. Adelmann, F. Chabuel, J. Simon, N. T. Pelekanos, G. Mula, B. Daudin, G. Feuillet, and H. Mariette, 2000, Appl. Phys. Lett. 77, 809.

Masumura, R. A., and G. Sines, 1970, J. Appl. Phys. 41, 3930.

Mateeva, E., P. Sutter, and M. G. Lagally, 1999, Appl. Phys. Lett. 74, 567.

Matthews, J. W., and A. E. Blakeslee, 1974, J. Cryst. Growth 27, 118.

Maximov M. V., 2003, private communication.

Medeiros-Ribeiro, G., A. M. Bratkowski, T. I. Kamins, D. A. A. Ohlberg, and R. S. Williams, 1998, Science 279, 353.

Medeiros-Ribeiro, G., T. I. Kamins, D. A. A. Ohlberg, and R. S. Williams, 1998, Phys. Rev. B 58, 3533.

Meduna, M., V. Holý, T. Roch, G. Bauer, O. G. Schmidt, and K. Eberl, 2001, J. Phys. D 34, A193.

Meixner, M., and E. Schöll, 2003, Phys. Rev. B 67, 121202(R). Meixner, M., E. Schöll, M. Schmidbauer, H. Raidt, and R. Köhler, 2001, Phys. Rev. B 64, 245307.

Miao, J., P. Charalambous, J. Kirz, and D. Sayre, 1999, Nature (London) 400, 342.
Michle, P., A. Kiraz, C. Becherand, W. V. Schoenfeld, P. M. Petroff, L. Zhang, E. Hu, and A. Imamoglu, 2000, Science 290, 2282.

Migliorato, M. A., A. G. Gullis, M. Fearn, and J. H. Jefferson, 2002, Phys. Rev. B 65, 115316.

Migliorato, M. A., L. R. Wilson, D. J. Mowbray, M. S. Skolnick, M. Al-Khafaji, A. G. Cullis, and M. Hopkinson, 2001, J. Appl. Phys. 90, 6374.

Miller, P. D., C.-M. Liu, W. L. Henstrom, J. M. Gibson, Y. Huang, P. Zhang, T. I. Kamins, D. P. Basile, and R. S. Williams, 1999, Appl. Phys. Lett. 75, 46.

Miyamura, M., K. Tachibana, and Y. Arakawa, 2002, Appl. Phys. Lett. 80, 3937.

Mo, Y.-M., D. E. Savage, B. S. Swartzentruber, and M. G. Lagally, 1990, Phys. Rev. Lett. 65, 1020.

Moll, N., M. Scheffler, and E. Pehlke, 1998, Phys. Rev. B 58, 4566.

Montalenti, F., 2004, in Proceedings of the NATO Advanced Research Workshop on Self-Assembled Quantum Dots, edited by D. Vvedensky (Kluwer Scientific, Dordrecht).

Moreau, E., I. Robert, J. M. Gérard, I. Abram, L. Manin, and V. Thierry-Mieg, 2001a, Appl. Phys. Lett. 79, 2865.

Moreau, E., I. Robert, L. Manin, V. Thierry-Mieg, J. M. Gérard, and I. Abram, 2001b, Phys. Rev. Lett. 87, 183601.

Moriarty, P., 2001, Rep. Prog. Phys. 64, 297.

Mühlberger, M., C. Schelling, G. Springholz, and F. Schäffler, 2003, Surf. Sci. 532-535, 721.

Murray, C. B., S. Sun, W. Gaschler, H. Doyle, T. A. Betley, and C. R. Kagan, 2001, IBM J. Res. Dev. 45, 47.

Murray, R., S. Malik, P. Siverns, D. Childs, C. Roberts, B. Joyce, and H. Davock, 1999, Jpn. J. Appl. Phys., Part 1 38, 496

Musgrave, M. J. P., and J. A. Pople, 1962, Proc. R. Soc. London, Ser. A 268, 474.

Myslivecek, J., C. Schelling, F. Schäffler, G. Springholz, P. Smilauer, J. Krug, and B. Voigtländer, 2002, Surf. Sci. 520, 193. Nelds, R. J., and R. M. Martin, 1984, Phys. Rev. B 30, 5390. Nötzel, R., 1996, Semicond. Sci. Technol. 11, 1365.

Ohlsson, B. J., M. T. Björk, M. H. Magnusson, K. Deppert, L. Samuelson, and L. R. Wallenberg, 2001, Appl. Phys. Lett. 79, 3335.

Ovidko, I. A., 2002, Phys. Rev. Lett. 88, 046103.

Ozasa, K., Y. Aoyagi, M. Iwaki, and H. Kurata, 2003, J. Appl. Phys. 94, 313.

Pan, E., 2002a, J. Appl. Phys. 91, 3785.

Pan, E., 2002b, J. Appl. Phys. 91, 6379.

Patriarche, G., A. Ougazzaden, and F. Glas, 2000, Appl. Phys. Lett. 76, 306.

Pehlke, E., and J. Tersoff, 1991, Phys. Rev. Lett. 67, 465.

Penev, E., P. Kratzer, and M. Scheffler, 2001, Phys. Rev. B 64, 085401.

Persson, J., M. Holm, and C. Pryor, 2003, Phys. Rev. B 67, 035320.

Petroff, P. M., A. Lorke, and A. Imamoglu, 2001, Phys. Today 54 (5) 46.

Petroff, P. M., and G. Medeiros-Ribeiro, 1996, MRS Bull. 21, 50.

Phang, Y. H., C. Teichert, M. G. Lagally, L. J. Peticolos, J. C. Bean, and E. Kasper, 1994, Phys. Rev. B 50, 14435.

Pidduck, A. J., D. J. Robbins, A. G. Cullis, W. Y. Leong, and A. M. Pitt, 1992, Thin Solid Films 222, 78.

Poon, T. W., S. Yip, P. S. Ho, and F. A. Abraham, 1990, Phys. Rev. Lett. 65, 2161. 
Priester, C., 2001, Phys. Rev. B 63, 153303.

Priester, C., and G. Grenet, 2001, Phys. Rev. B 64, 125312.

Proietti, M. G., H. Renevier, J. L. Hodeau, J. Garcia, J. F. Berar, and P. Wolfers, 1999, Phys. Rev. B 59, 5479.

Pryor, C., J. Kim, L. W. Wang, A. J. Williamson, and A. Zunger, 1998, J. Appl. Phys. 83, 2548.

Qin, X. R., B. S. Schwartzentruber, and M. G. Lagally, 2000a, Phys. Rev. Lett. 85, 3660.

Qin, X. R., B. S. Schwartzentruber, and M. G. Lagally, 2000b, Phys. Rev. Lett. 84, 4645.

Raiteri, P., D. B. Migas, L. Miglio, A. Rastelli, and H. von Kaenel, 2002, Phys. Rev. Lett. 88, 256103.

Rastelli, A., 2004, private communication.

Rastelli, A., M. Kummer, and H. von Kaenel, 2001, Phys. Rev. Lett. 87, 256101.

Rastelli, A., M. Kummer, and H. von Kaenel, 2002, Physica E (Amsterdam) 13, 1008.

Rastelli, A., E. Mueller, and H. von Kaenel, 2002, Appl. Phys. Lett. 80, 1438.

Rastelli, A., and H. von Känel, 2002, Surf. Sci. 515, L493.

Rastelli, A., and H. von Känel, 2003, Surf. Sci. 532-535, 769.

Rastelli, A., H. von Kaenel, G. Albini, P. Raiteri, D. B. Migas, and L. Miglio, 2003, Phys. Rev. Lett. 90, 216104.

Rastelli, A., H. von Känel, B. J. Spencer, and J. Tersoff, 2003, Phys. Rev. B 68, 115301.

Rauscher, M., R. Paniago, H. Metzger, Z. Kovats, J. Domke, J. Peisl, H.-M. Pfannes, J. Schulze, and I. Eisele, 1999, J. Appl. Phys. 86, 6763.

Redl, F. X., K.-M. Cho, C. B. Murray, and S. O'Brien, 2003 , Nature (London) 423, 968.

Regelman, D. V., E. Dekel, D. Gershoni, E. Ehrenfreund, A. J. Williamson, J. Shumway, A. Zunger, W. V. Schoenfeld, and P. M. Petroff, 2001, Phys. Rev. B 64, 165301.

Renaud, G., R. Lazzari, C. Revenant, A. Barbier, M. Noblet, O. Ulrich, F. Leroy, J. Jupille, Y. Borensztein, C. R. Henry, J. P. Deville, F. Scheurer, J. Mane-Mane, and O. Fruchart, 2003, Science 300, 1416.

Ribbat, C., R. L. Sellin, I. Kaiander, F. Hopfer, N. N. Ledentsov, D. Bimberg, A. R. Kovsh, V. M. Ustinov, A. E. Zhukov, and M. V. Maximov, 2003, Appl. Phys. Lett. 82, 952.

Robey, S. W., 2002, Phys. Rev. B 65, 115306.

Robinson, I. K., and D. J. Tweet, 1992, Rep. Prog. Phys. 55, 599.

Robinson, I. K., I. A. Vartanyants, G. J. Williams, M. A. Pfeifer, and J. A. Pitney, 2001, Phys. Rev. Lett. 87, 195505.

Roch, T., V. Holý, A. Hesse, J. Stangl, T. Fromherz, G. Bauer, T. H. Metzger, and S. Ferrer, 2002, Phys. Rev. B 65, 245324.

Roh, Cheong Hyun, Young Ju Park, Kwang Moo Kim, Young Min Park, Fun Kyu Kim, and Kwang Bo Shim, 2001, J. Cryst. Growth 226, 1.

Romanov, A. E., G. E. Beltz, W. T. Fischer, P. M. Petroff, and J. S. Speck, 2001, J. Appl. Phys. 89, 4523.

Ronda, A., M. Abdallah, J. Gay, J. Stettner, and I. Berbezier, 2000, Appl. Surf. Sci. 162-163, 576.

Rosenauer, A., U. Fischer, D. Gerthsen, and A. Förster, 1997, Appl. Phys. Lett. 71, 3868.

Rosenauer, A., U. Fischer, D. Gerthsen, and A. Förster, 1998, Ultramicroscopy 72, 121.

Rosenauer, A., and D. Gerthsen, 1999, Ultramicroscopy 76, 49. Rosenauer, A., D. Gerthsen, D. van Dyck, M. Artzberger, G. Boehm, and G. Abstreiter, 2001, Phys. Rev. B 64, 245334.

Rosenauer, A., W. Oberst, D. Litvinov, D. Gerthsen, A. Förster, and R. Schmidt, 2000, Phys. Rev. B 61, 8276.
Ross, F. M., J. Tersoff, and R. M. Tromp, 1998, Phys. Rev. Lett. 80, 984.

Ross, F. M., R. M. Tromp, and M. C. Reuter, 1999, Science 286, 1931.

Rost, M., P. Smilauer, and J. Krug, 1996, Surf. Sci. 369, 393.

Rudd, R. E., G. A. D. Briggs, A. P. Sutton, G. MedeirosRibeiro, and R. S. Williams, 2003, Phys. Rev. Lett. 90, 146101. Sahtori, C., D. Fattal, J. Vučković, G. S. Solomon, and Y. Yamamoto, 2002, Nature (London) 419, 594.

Saito, H., K. Nishi, and S. Sugou, 1998, Appl. Phys. Lett. 73, 2472.

Saito, T., and Y. Arakawa, 2002, Physica E (Amsterdam) 15, 169.

Saitta, A. M., F. Buda, G. Fiumara, and P. V. Giaquinta, 1996, Phys. Rev. Lett. 53, 1446.

Sakamoto, K., H. Matsuhata, M. O. Tanner, D. Wang, and K. L. Wang, 1998, Thin Solid Films 321, 55.

Samuelson, L., 2003, Mater. Today 6, 22.

Schelling, C., M. Mühlberger, G. Springholz, and F. Schäffler, 2001, Phys. Rev. B 64, 041301(R).

Schelling, C., G. Springholz, and F. Schäffler, 1999, Phys. Rev. Lett. 83, 995.

Schikora, D., S. Schwedhelm, D. J. As, K. Lischka, D. Litvinov, A. Rosenauer, D. Gerthsen, M. Strassburg, A. Hoffmann, and D. Bimberg, 2000, Appl. Phys. Lett. 76, 418.

Schmidbauer, M., 2004, Springer Tracts in Modern Physics 199, 1 (Springer, Berlin).

Schmidbauer, M., M. Hanke, and R. Köhler, 2002, Cryst. Res. Technol. 36, 1 .

Schmidbauer, M., F. Hatami, M. Hanke, P. Schaefer, K. Braune, W. T. Masselink, R. Köhler, and M. Ramsteiner, 2002, Phys. Rev. B 65, 125320.

Schmidbauer, M., R. Opitz, T. Wiebach, and R. Köhler, 2001, Phys. Rev. B 64, 195316.

Schmidbauer, M., T. Wiebach, H. Raidt, M. Hanke, R. Köhler, and H. Wawra, 1998, Phys. Rev. B 58, 10523.

Schmidt, O. G., U. Denker, S. Christiansen, and F. Ernst, 2002, Appl. Phys. Lett. 81, 2614.

Schmidt, O. G., U. Denker, K. Eberl, O. Kienzle, and F. Ernst, 2000, Appl. Phys. Lett. 77, 2509.

Schmidt, O. G., and K. Eberl, 2000, Phys. Rev. B 61, 13721.

Schmidt, O. G., and K. Eberl, 2001, IEEE Trans. Electron Devices 48, 1175.

Schmidt, O. G., K. Eberl, and Y. Rau, 2000, Phys. Rev. B 62, 16715.

Schmidt, O. G., N. Y. Jin-Phillip, C. Lange, U. Denker, K. Eberl, R. Schreiner, H. Graebeldinger, and H. Schweitzer, 2000, Appl. Phys. Lett. 77, 4139.

Schmidt, O. G., S. Kiravittaya, Y. Nakamura, H. Heidemeyer, R. Songmuang, C. Mueller, N. Jin-Phillip, K. Eberl, H. Wawra, S. Christiansen, H. Graebeldinger, and H. Schweizer, 2002, Surf. Sci. 514, 10.

Schmidt, O. G., C. Lange, K. Eberl, O. Kienzle, and F. Ernst, 1997, Appl. Phys. Lett. 71, 2340.

Schmidt, O. G., Y. M. Manz, and K. Eberl, 2002, in NanoOptoelectronics, edited by M. Grundmann (Springer, Berlin), pp. 339-352.

Schuelli, T. U., M. Sztucki, V. Chamard, T. H. Metzger, and D. Schuh, 2002, Appl. Phys. Lett. 81, 448.

Schülli, T. U., J. Stangl, Z. Zhong, R. T. Lechner, M. Sztucki, T. H. Metzger, and G. Bauer, 2003, Phys. Rev. Lett. 90, 066105. Schwoebel, R. L., and E. J. Shipsey, 1966, J. Appl. Phys. 37, 3682 . 
Seifert, W., N. Carlsson, M. Miller, M. E. Pistol, L. Samuelson, and L. R. Reine Wallenberg, 1996, Prog. Cryst. Growth Charact. Mater. 33, 423.

Sellers, I. R., H. Y. Liu, M. Hopkinson, D. J. Mowbray, and M. S. Skolnick, 2003, Appl. Phys. Lett. 83, 4710.

Seok, J. H., and J. Y. Kim, 2001, Appl. Phys. Lett. 78, 3124.

Seta, M. D., G. Capellini, F. Evangelisti, and C. Spinella, 2002, J. Appl. Phys. 92, 614.

Seufert, J., G. Bacher, M. Scheibner, A. Forchel, S. Lee, M. Dobrowolska, and J. K. Furdyna, 2002, Phys. Rev. Lett. 88, 027402.

Seufert, J., G. Bacher, H. Schoemig, A. Forchel, L. Hansen, G. Schmidt, and L. W. Molenkamp, 2004, Phys. Rev. B 69, 035311.

Shchekin, O. B., and D. G. Deppe, 2002, Appl. Phys. Lett. 80, 3277.

Shchukin, V., N. N. Ledentsov, and D. Bimberg, 2003, Epitaxy of Nanostructures (Springer, Berlin/Heidelberg).

Shchukin, V. A., and D. Bimberg, 1999, Rev. Mod. Phys. 71, 1125.

Shchukin, V. A., D. Bimberg, V. G. Malyshkin, and N. N. Ledentsov, 1998, Phys. Rev. B 57, 12262.

Shchukin, V. A., N. N. Ledentsov, A. Hoffmann, D. Bimberg, I. P. Soshnikov, B. V. Volovik, V. M. Ustinov, D. Litvinov, and D. Gerthsen, 2001, Phys. Status Solidi B 224, 503.

Shchukin, V. A., N. N. Ledentsov, P. S. Kopev, and D. Bimberg, 1995, Phys. Rev. Lett. 75, 2968.

Shilkrot, L. E., and D. J. Srolovitz, 1997, Phys. Rev. B 55, 4737.

Shilkrot, L. E., D. J. Srolovitz, and J. Tersoff, 2000, Appl. Phys. Lett. 77, 304.

Shiryaev, S., F. Jensen, J. L. Hansen, J. W. Petersen, and A. N. Larsen, 1997, Phys. Rev. Lett. 78, 503.

Skolnick, M. S. and D. J.Mowbray, 2004, Annual Rev. Mater. Res. 34, 181.

Songmuang, R., S. Kiravittaya, and O. G. Schmidt, 2003, Appl. Phys. Lett. 82, 2892.

Sonnet, P., and P. C. Kelires, 2002, Phys. Rev. B 66, 205307.

Spencer, B. J., and D. I. Meiron, 1994, Acta Metall. Mater. 42, 3629.

Spencer, B. J., and J. Tersoff, 2000, Appl. Phys. Lett. 77, 2533. Spencer, B. J., and J. Tersoff, 2001, Phys. Rev. B 63, 205424.

Spencer, B. J., P. W. Voorhes, and J. Tersoff, 2001, Phys. Rev. B 64, 235318.

Spencer, B. J., P. W. Voorhees, and S. H. Davis, 1993, J. Appl. Phys. 73, 4955.

Springholz, G., V. Holý, P. Mayer, M. Pinczolits, A. Raab, R. T. Lechner, G. Bauer, H. Kang, and L. Salamanca-Riba, 2002, Mater. Sci. Eng., B 88, 143.

Springholz, G., V. Holý, M. Pinczolits, and G. Bauer, 1998, Science 282, 734.

Springholz, G., M. Pinczolits, V. Holý, P. Mayer, K. Wiesauer, T. Roch, and G. Bauer, 2000b, Surf. Sci. 454-456, 657.

Springholz, G., M. Pinczolits, V. Holý, S. Zerlauth, I. Vavra, and G. Bauer, 2001, Physica E (Amsterdam) 9, 657.

Springholz, G., M. Pinczolits, P. Mayer, V. Holý, G. Bauer, H. H. Kang, and L. Salamanca-Riba, 2000a, Phys. Rev. Lett. 84, 4669.

Springholz, G., T. Schwarzl, W. Heiss, G. Bauer, M. Aigle, H. Pascher, and I. Vavra, 2001, Appl. Phys. Lett. 79, 1225.

Springholz, G., J. Stangl, M. Pinczolits, V. Holý, P. Mikulik, P. Mayer, K. Wiesauer, G. Bauer, D. Smilgies, H. H. Kang, and L. Salamanca-Riba, 2000, Physica E (Amsterdam) 7, 870.

Srolovitz, D. J., 1989, Acta Metall. 37, 621.
Stangl, J., A. Daniel, V. Holý, T. Roch, G. Bauer, I. Kegel, T. H. Metzger, T. Wiebach, O. Schmidt, and K. Eberl, 2001, J. Appl. Phys. 79, 1474.

Stangl, J., A. Hesse, V. Holý, Z. Zhong, G. Bauer, U. Denker, and O. G. Schmidt, 2003, Appl. Phys. Lett. 82, 2251.

Stangl, J., V. Holý, P. Mikulik, G. Bauer, I. Kegel, T. H. Metzger, O. G. Schmidt, C. Lange, and K. Eberl, 1999, J. Appl. Phys. 74, 3785.

Stangl, J., V. Holý, T. Roch, A. Daniel, G. Bauer, J. Zhu, K. Brunner, and G. Abstreiter, 2000, Phys. Rev. B 62, 7229.

Stangl, J., T. Roch, G. Bauer, I. Kegel, T. H. Metzger, O. Schmidt, K. Eberl, O. Kienzle, and F. Ernst, 2000, Appl. Phys. Lett. 77, 3953.

Steinfort, A. J., P. M. L. O. Sholte, A. Ettema, F. Tuinstra, M. Nielsen, E. Landemark, D.-M. Smilgies, R. Feidenhansl, G. Falkenberg, L. Seehofer, and R. L. Johnson, 1996, Phys. Rev. Lett. 77, 2009.

Stepanov, S. A., E. A. Kondrashkina, M. Schmidbauer, R. Köhler, J.-U. Pfeiffer, T. Jach, and A. Y. Souvorov, 1996, Phys. Rev. B 54, 8150.

Stepanov, S. A., and S. K. Sinha, 2000, Phys. Rev. B 61, 15302. Stillinger, F. H., and T. A. Weber, 1985, Phys. Rev. B 31, 5262. Stoffel, M., U. Denker, and O. G. Schmidt, 2003, Appl. Phys. Lett. 82, 3236.

Stranski, I. N., and L. Krastanow, 1938, Sitzungsber. Akad. Wiss. Wien, Math.-Naturwiss. Kl., Abt. 2B 146, 797.

Strassburg, M., T. Deniozou, R. Heitz, U. W. Pohl, D. Bimberg, D. Litvinov, A. Rosenauer, D. Gehrtsen, S. Schwedhelm, K. Lischka, and D. Schikora, 2000, Appl. Phys. Lett. 76, 685.

Strassburg, M., A. V. Kutzer, U. W. Pohl, A. Hoffmann, I. Broser, N. N. Ledentsov, D. Bimberg, A. Rosenauer, U. Fischer, D. Gehrtsen, I. L. Krestnikov, M. V. Maximov, P. S. Kop'ev, and Zh. L. Alferov, 1998, Appl. Phys. Lett. 72, 942. Su, X., R. K. Kalia, A. Nakano, P. Vashishta, and A. Madhukar, 2001, Appl. Phys. Lett. 79, 4577.

Su, X., R. K. Kalia, A. Nakano, P. Vashishta, and A. Madhukar, 2003, J. Appl. Phys. 94, 6762.

Sullivan, J. S., E. Mateeva, H. Evans, D. E. Savage, and M. G. Lagally, 1999, J. Vac. Sci. Technol. A 17, 2345.

Sutter, P., and M. G. Lagally, 1998, Phys. Rev. Lett. 81, 3471. Sutter, P., and M. C. Lagally, 2000, Phys. Rev. Lett. 84, 4637.

Sutter, P., E. Mateeva-Sutter, and L. Vescan, 2001, Appl. Phys. Lett. 78, 1736.

Sutter, P., I. Schick, W. Ernst, and E. Sutter, 2003, Phys. Rev. Lett. 91, 176102.

Tan, P. H., K. Brunner, D. Bougeard, and G. Abstreiter, 2003, Phys. Rev. B 68, 125302.

Teichert, C., C. Hofer, K. Lyutovich, M. Bauer, and E. Kasper, 2000, Thin Solid Films 380, 25.

Teichert, C., C. Hofer, K. Lyutovich, M. Bauer, and E. Kasper, 2002, Phys. Rep. 365, 335.

Teichert, C., L. J. Peticolas, J. C. Bean, J. Tersoff, and M. G. Lagally, 1996, Phys. Rev. B 53, 16334.

Tersoff, J., 1988, Phys. Rev. B 38, 9902.

Tersoff, J., 1989, Phys. Rev. B 39, 5566.

Tersoff, J., 1996, Phys. Rev. Lett. 77, 2017.

Tersoff, J., 1998, Phys. Rev. Lett. 81, 3183.

Tersoff, J., 2003, Appl. Phys. Lett. 83, 353.

Tersoff, J., and F. K. LeGoues, 1994, Phys. Rev. Lett. 72, 3570.

Tersoff, J., Y. H. Phang, Z. Zhang, and M. G. Lagally, 1995, Phys. Rev. Lett. 75, 2730.

Tersoff, J., B. Spencer, A. Rastelli, and H. von Känel, 2002, 
Phys. Rev. Lett. 89, 196104.

Tersoff, J., C. Teichert, and M. G. Lagally, 1996, Phys. Rev. Lett. 76, 1675.

Tersoff, J., A. W. D. van der Gon, and R. M. Tromp, 1994, Phys. Rev. Lett. 72, 266.

Tillmann, K., and A. Foerster, 2000, Thin Solid Films 368, 93. Timoshenko, S. P., and J. N. Goodier, 1971, Theory of Elasticity (McGraw-Hill, New York).

Tischler, J. Z., J. D. Budai, D. E. Jesson, G. Eres, and P. Zschack, 1995, Phys. Rev. B 51, 10947.

Titova, L. V., J. K. Furdyna, M. Dobrowolska, S. Lee, T. Topuria, P. Moeck, and N. D. Browning, 2002, Appl. Phys. Lett. 80, 1237.

Tromp, R. M., and M. C. Reuter, 1992, Phys. Rev. Lett. 68, 820. Tromp, R. M., F. M. Ross, and M. C. Reuter, 2000, Phys. Rev. Lett. 84, 4641.

Twesten, R. D., D. M. Follstaedt, S. R. Lee, E. D. Jones, J. L. Reno, J. M. Millunchick, A. G. Norman, S. P. Ahrenkiel, and A. Mascarenhas, 1999, Phys. Rev. B 60, 13619.

Ulbrich, N., J. Bauer, G. Scarpa, R. Boy, D. Schuh, G. Abstreiter, S. Schmult, and W. Wegscheide, 2003, Appl. Phys. Lett. 83, 1530.

Usami, N., Y. Araki, Y. Ito, M. Miura, and Y. Shiraki, 2000a, Appl. Phys. Lett. 76, 3723.

Usami, N., M. Miura, Y. Ito, Y. Araki, and Y. Shiraki, 2000b, Appl. Phys. Lett. 77, 217.

Ustinov, V. M., and A. E. Zhukov, 2002, Semicond. Sci. Technol. 15, R41.

Ustinov, V. M., A. E. Zukov, A. Yu. Egorov, and N. A. Maleen, 2003, Quantum Dot Lasers (Oxford University Press, Oxford).

Vailionis, A., B. Cho, G. Glass, P. Desjardins, D. G. Cahill, and J. E. Greene, 2000, Phys. Rev. Lett. 85, 3672.

Vanderbilt, D., 1986, Surf. Sci. 268, L300.

Vandervelde, T. E., P. Kumar, T. Kobayashi, J. L. Gray, T. Pernell, J. A. Floro, R. Hull, and J. C. Bean, 2003, Appl. Phys. Lett. 83, 5205.

Vartanyants, I. A., and I. K. Robinson, 2003, J. Synchrotron Radiat. 10, 409.

Venezuela, P., J. Tersoff, J. A. Floro, E. Chason, D. M. Follstaedt, Feng Liu, and M. G. Lagally, 1999, Nature (London) 397, 678.

Vescan, L., and T. Stoica, 2002, J. Appl. Phys. 91, 10119.

Vescan, L., T. Stoica, B. Holländer, A. Nassiopoulou, A. Olzierski, I. Raptis, and E. Sutter, 2003, Appl. Phys. Lett. 82, 3517.

Villain, J., 1991, J. Phys. I 1, 19.

Voigtländer, B., 2001, Surf. Sci. Rep. 43, 127.

Wachowiak, A., J. Wiebe, M. Bode, O. Pietzsch, M. Morgenstern, and R. Wiesendanger, 2002, Science 298, 577.

Walther, T., A. G. Cullis, D. J. Norris, and M. Hopkinson, 2001, Phys. Rev. Lett. 86, 2381.

Wasserman, D., S. A. Lyon, M. H. A. Maciel, and J. F. Ryan, 2003, Appl. Phys. Lett. 83, 5050.
Wiebach, T., M. Schmidbauer, M. Hanke, H. Raidt, R. Köhler, and H. Wawra, 2000, Phys. Rev. B 61, 5571.

Williams, A. A., J. M. C. Thornton, J. E. Macdonald, R. G. van Silfhout, J. F. van der Veen, M. S. Finney, A. D. Johnson, and C. Norris, 1991, Phys. Rev. B 43, 5001.

Williams, G. J., M. A. Pfeifer, I. A. Vartanyants, and I. K. Robinson, 2003, Phys. Rev. Lett. 90, 175501.

Woggon, U., D. Miller, F. Kalina, B. Gerlach, D. Kayser, K. Leonardi, and D. Hommel, 2003, Phys. Rev. B 67, 045204.

Wolf, S. A., D. D. Awschalom, R. A. Buhrman, J. M. Daughton, S. von Molnár, M. L. Roukes, A. Y. Chtchelkanova, and D. M. Treger, 2001, Science 294, 1488.

Wortman, J. J., and R. A. Evans, 1965, J. Appl. Phys. 36, 153. Xie, Q., A. Madhukar, P. Chen, and N. P. Kobayashi, 1995, Phys. Rev. Lett. 75, 2542.

Xie, Y. H., G. H. Gilmer, C. Roland, P. J. Silverman, S. K. Buratto, J. Y. Cheng, E. A. Fitzgerald, A. R. Kortan, S. Schuppler, M. A. Marcus, and P. H. Citrin, 1994, Phys. Rev. Lett. 73, 3006.

Xue, Q., T. Ogino, H. Kiyama, Y. Hasegawa, and T. Sakurai, 1997, J. Cryst. Growth 175-176, 174.

Xue, Q. K., and T. Sakurai, 1998, Phys. Rev. B 57, R6862.

Yamaguchi, K., Y. Saito, and R. Ohtsubo, 2002, Appl. Surf. Sci. 190, 212.

Yang, B., 2003, J. Appl. Phys. 92, 3704.

Yang, B., and V. K. Tewary, 2003, Phys. Rev. B 68, 035301.

Yang, T., S. Kohmoto, H. Nakamura, and K. Asakawa, 2003, J. Appl. Phys. 93, 1190.

Ye, Z., J. C. Campbell, Z. Chen, E. T. Kim, and A. Madhukar, 2002, J. Appl. Phys. 92, 7462.

Yin, M. T., and M. L. Cohen, 1982, Phys. Rev. B 26, 5668.

Yoon, S., Y. Moon, T.-W. Lee, E. Yoon, and Y. D. Kim, 1999, Appl. Phys. Lett. 74, 2029.

Yuan, Z., B. E. Kardynal, R. M. Stevenson, A. J. Shields, C. J. Lobo, K. Cooper, N. S. Beattie, D. A. Ritchie, and M. Pepper, 2002, Science 295, 102.

Zangwill, A., 1988, Physics at Surfaces (Cambridge University, Cambridge, England).

Zhang, K., C. Heyn, W. Hansen, T. Schmidt, and J. Falta, 2000, Appl. Phys. Lett. 76, 2229.

Zhang, Y. W., S. J. Xu, and C. H. Chiu, 1999, Appl. Phys. Lett. 74, 1809.

Zhi, D., H. Davock, R. Murray, C. Roberts, T. S. Jones, D. W. Pashley, P. J. Goodhew, and B. A. Joyce, 2001, J. Appl. Phys. 89, 2079.

Zhong, Z. and G. Bauer, 2004, Appl. Phys. Lett. 84, 1922.

Zhong, Z., A. Halilovic, T. Fromherz, F. Schäffler, and G. Bauer, 2003, Appl. Phys. Lett. 82, 4779.

Zrenner, A., E. Beham, S. Stufler, F. Findeis, M. Bichler, and G. Abstreiter, 2002, Nature (London) 418, 612.

Zunger, A., 1994, in Handbook of Crystal Growth, edited by D. T. J. Hurle (Elsevier, Amsterdam), Vol. 3, 998.

Zwiller, V., T. Aichele, W. Seifert, J. Persson, and O. Benson, 2003, Appl. Phys. Lett. 82, 1509. 